\title{
Transport in Hamilton-Systemen: Von der Klassik zur Quantenmechanik
}

\section{Dissertation}

zur Erlangung des Doktorgrades

der Mathematisch-Naturwissenschaftlichen Fakultäten der Georg-August-Universität zu Göttingen

vorgelegt von

\section{Lars Hufnagel}

aus Lippstadt

Göttingen 2001 
D7

Referent:

Koreferent:
Prof. Dr. Theo Geisel

Prof. Dr. Kurt Schönhammer

Tag der mündlichen Prüfung: 


\section{Inhaltsverzeichnis}

1 Einleitung $\quad 7$

2 Hamiltonsches Chaos $\quad 13$

2.1 Dynamische Systeme . . . . . . . . . . . . . . . 13

2.1.1 Grundbegriffe ........................... 13

2.1.2 Poincaré-Abbildung . . . . . . . . . . . . . . 14

2.2 Hamiltonsche Dynamik . . . . . . . . . . . . . . 15

2.2.1 Hamilton-Systeme . . . . . . . . . . . . . . . 15

2.2.2 Erweiterter Phasenraum . . . . . . . . . . . . . . 16

2.2 .3 Integralinvarianten . . . . . . . . . . . . . . 17

2.2 .4 Erhaltungsgrößen . . . . . . . . . . . . . . 17

2.2.5 Poincaré-Abbildung Hamiltonscher Systeme . . . . . . . . . . . 18

2.3 Vom integrablen zum chaotischen Phasenraum . . . . . . . . . . . . 19

2.3.1 Störung integrabler Systeme . . . . . . . . . . . . . . . . 19

2.3 .2 KAM-Theorie ...................... 20

2.3.3 Chaotische Dynamik .................. 23

2.4 Transport in gemischten Systemen . . . . . . . . . . . . . 25

2.4 .1 Phasenraumfluss . . . . . . . . . . . . . . 25 
2.4 .2 Partielle Transportbarrieren . . . . . . . . . . . . 26

2.4.3 Markov-Modell zum Phasenraumtransport . . . . . . . . . . . 28

2.5 Quantenmechanik gekickter Systeme . . . . . . . . . . . 30

3 Chirikovs siebtes Gesetz 33

3.1 Kritischer goldener Torus . . . . . . . . . . . . . . . 33

3.2 Insel-um-Insel . . . . . . . . . . . . . . . . . . . . . . . 37

3.3 Zusammenfassung und Ausblick . . . . . . . . . . . . . 41

4 Hierarchische Eigenzustände $\quad 43$

4.1 Eine neue Klasse von Eigenzuständen . . . . . . . . . . . . . . 43

4.2 Der Anteil der hierarchischen Eigenzustände . . . . . . . . . . . . 45

4.2.1 Einfluss auf die Eigenfunktionen . . . . . . . . . . . . . 45

4.2 .2 Einfluss auf das Spektrum . . . . . . . . . . . . . . 46

4.3 Quantenmechanik des Kettenmodells . . . . . . . . . . . . 48

4.4 Quantenmechanische Modellierung des Phasenraums . . . . . . . 50

4.5 Zusammenfassung und Ausblick . . . . . . . . . . . . . 52

5 Leitwertfluktuationen $\quad 53$

5.1 Transport mesoskopischer Systeme . . . . . . . . . . . . 53

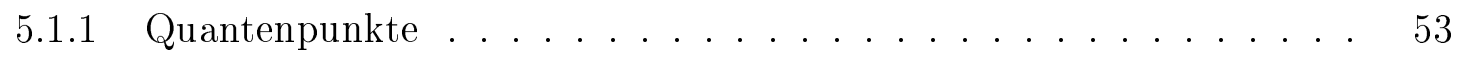

5.1 .2 Landauer Formel . . . . . . . . . . . . . . . . 54

5.2 Leitwertfluktuationen . . . . . . . . . . . . . . . 55

5.2 .1 Universelle Leitwertfluktuationen . . . . . . . . . . . . 55

5.2 .2 Fraktale Leitwertfluktuationen . . . . . . . . . . . . 56

5.2 .3 Isolierte Resonanzen . . . . . . . . . . . . . . . . . 58 
$5.3 \quad$ Fraktal oder isoliert? . . . . . . . . . . . . . . . . 59

5.4 Graphenmodell für den gemischten Phasenraum . . . . . . . . . . . . . 61

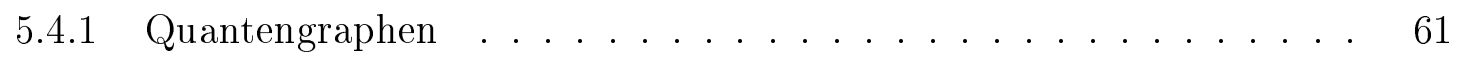

5.4.2 Hierarchische Quantengraphen ............... 64

5.4 .3 Leitwerte für Quantengraphen . . . . . . . . . . . . . . . 66

5.5 Zusammenfassung und Ausblick . . . . . . . . . . . . . . 69

6 Kreuzförmiger Transport $\quad 71$

6.1 Das doppelt gekickte Harper-Modell . . . . . . . . . . . . . 72

6.2 Numerische Behandlung . . . . . . . . . . . . . 73

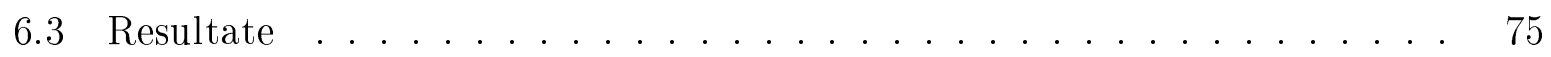

6.4 Zusammenfassung . . . . . . . . . . . . . . 78

$\begin{array}{lll}7 & \text { Superballistischer Transport } & 79\end{array}$

7.1 Superballistischer Transport . . . . . . . . . . . . . . 80

7.2 Ein einfaches Modell . . . . . . . . . . . . . . . . . 81

7.3 Numerische Behandlung . . . . . . . . . . . . . 82

7.3.1 Anderson-Modell . . . . . . . . . . . . . . . . . 84

$7.3 .2 \quad$ BRM-Modell . . . . . . . . . . . . . . 85

7.3.3 Fibonacci-Modell . . . . . . . . . . . . . . . 86

7.4 Zusammenfassung . . . . . . . . . . . . . . 87

$\begin{array}{llr}8 & \text { Zusammenfassung } & 89\end{array}$ 


\section{Kapitel 1}

\section{Einleitung}

Seit der Entdeckung des Elektrons 1897 durch J. J. Thompson und Drudes kinetischer Gastheorie für die Elektronenbewegung werden Transporteigenschaften von Elektronen in Festkörpern intensiv experimentell wie theoretisch untersucht. Faszinierende Phänomene, wie z.B. Supraleitung und Quantenhalleffekt, wurden entdeckt. Aber nicht nur die Entdeckung interessanter Effekte nährt die Erforschung elektronischer Transporteigenschaften, auch ihre Anwendungen sind aus dem täglichen Leben nicht mehr wegzudenken, wie die Omnipräsenz des Transistors zeigt. Ein beeindruckender Aspekt der Halbleiterentwicklung ist der Geschwindigkeit der Miniaturisierung, die mit der Entwicklung des Transistors seit

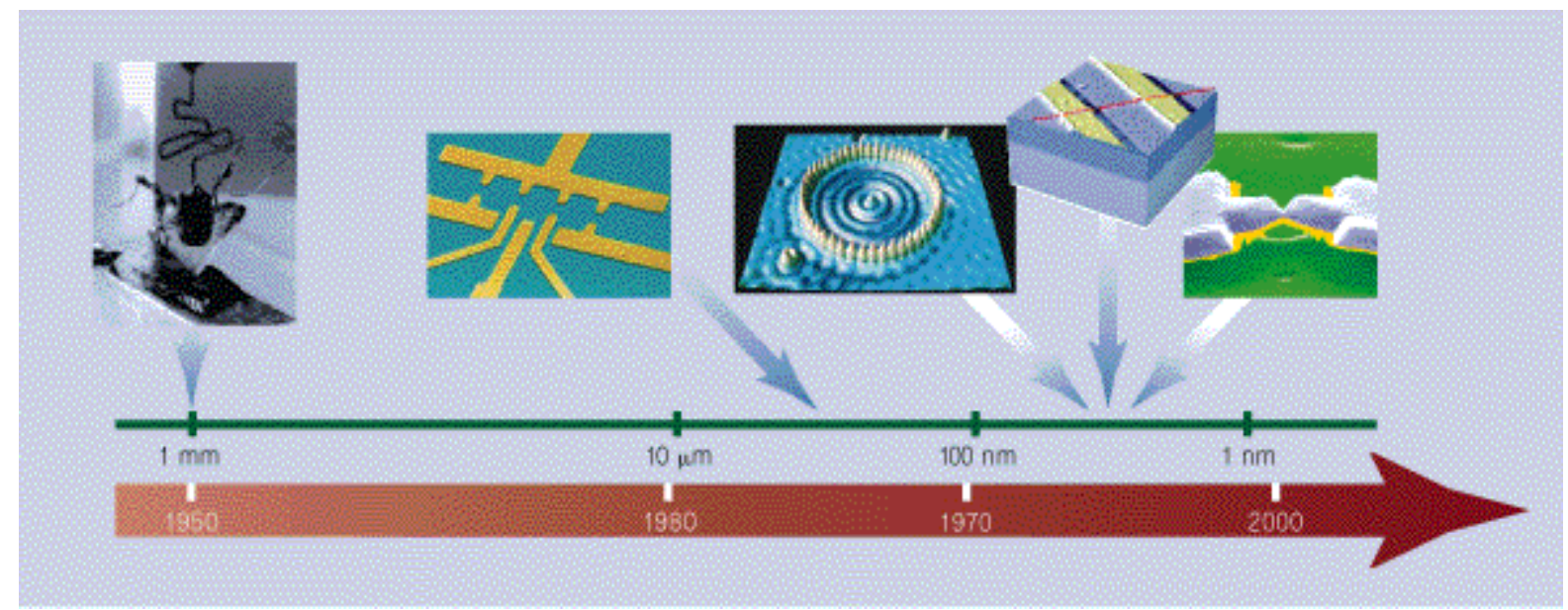

Abbildung 1.1: Miniaturisierung von elektronischen Komponenten. Die technologisch erzielten Längenskalen sind seit 1950 von einigen Millimetern bis zu atomaren Abmessungen geschrumpft. Die Bilder zeigen von links nach rechts: den ersten Transistor, einen Quantenpunkt, 48 Eisenatome aufgereiht zu einem Kreis (quantum corral), einen "carbon-nanotube" Transistor und einen atomaren Punktkontakt [1]. 
nun mehr als 50 Jahren voranschreitet. Die Extrapolation dieses Trends auf die nächsten 10 bis 15 Jahren prognostiziert, dass die Abmessungen der Untereinheiten, aus denen sich ein Chip zusammensetzt, nur wenige Nanometer betragen werden: so werden die Verbindungsleitungen nur 10 Atome breit sein.

Zunächst scheinen die größten Herausforderungen experimenteller bzw. technologischer Natur zu sein. Aber auch von theoretischer Seite ist diese Nanowelt äußerst interessant, da hier zwei Beschreibungen aufeinander treffen: die makroskopische und mikroskopische. Auf makroskopischen Skalen bildet die klassische Mechanik eine geschlossene Beschreibung physikalischer Größen, wie z.B. die Bewegung von Himmelskörpern. Die Phänomene auf mikroskopischen Skalen, wie z.B. Atome oder Moleküle, werden durch die Quantenmechanik beschrieben. Obwohl die Quantenmechanik die klassische Mechanik als Grenzfall enthält, wäre eine quantenmechanische Berechnung der Planetenbewegungen höchst unpraktikabel. Zwischen diesen Skalen liegt der Bereich der mesoskopischen Systeme, wo eine quantenmechanische Beschreibung unerlässlich ist, aber gleichzeitig Spuren der klassischen Dynamik sichtbar sind. Oftmals sind es gerade diese klassischen Aspekte, die ein intuitives Verständnis mesoskopischer Phänomene erlauben. Es liegt im Herzen des Gebiets Quantenchaos, eine Verbindung herzustellen zwischen der chaotischen klassischen Dynamik und den Eigenschaften des korrespondierenden quantenmechanischen Systems. Dabei lag der Schwerpunkt der Untersuchungen bisher bei vollständig chaotischen Systemen. Generische Hamilton-Systeme zeichnen sich durch einen gemischten Phasenraum aus, in dem Bereiche regulärer und chaotischer Dynamik eng miteinander verzahnt sind. Der Schwerpunkt dieser Arbeit liegt bei der Untersuchung von Quantensignaturen solcher gemischter Systeme.

Die grundlegenden Arbeiten von Henri Poincaré vor etwa hundert Jahren markieren die Anfänge des Gebiets des Hamiltonschen Chaos [2]. Dabei ging es ihm nicht um das exakte Lösen der Bewegungsgleichungen, sondern um ein qualitatives Verständnis der klassischen Dynamik: Was geschieht mit den invarianten Tori eines integrablen Hamilton-Systems unter einer kleinen Störung? Schon Poincaré erkannte, dass der entstehende Phasenraum eine komplexe Struktur aufweist, jedoch gelang der Beweis erst 50 Jahre später. Nach Arbeiten von Birkhoff und Hopf [3-5] konnten Kolmogorov, Arnold und Moser [6-10] in den sechziger Jahren zeigen, dass nicht alle invarianten Tori durch die Störung zerbrechen. Darauf aufbauend gelang Aubry [11,12] und Mather [13] eine komplette Klassifizierung des Verhaltens invarianter Tori unter einer Störung: Tori mit irrationaler Windungszahl bleiben entweder erhalten oder bilden sogenannte Cantori. Tori mit rationaler Windungszahl zerbrechen sofort. Dabei entsteht eine Kette von elliptischen und hyperbolischen Fixpunkten. Letztere sind die Keimzellen der chaotischen Dynamik in Hamilton-Systemen, wie Smale [14] zeigen konnte. Zusammenfassend stellt sich das Bild heute folgendermaßen dar: Eingebettet in eine chaotische invariante Komponente sind Inseln regulärer Dynamik. Diese Inseln sind von Inselketten umgeben, welche wiederum Inseln als Satelliten haben, usw. Der Phasenraum eines generischen Hamilton-Systems enthält also chaotisch und reguläre Komponenten, weshalb man von Systemen mit gemischtem Phasenraum spricht. 
Die chaotische Dynamik im gemischten Phasenraum und in idealisierten vollständig chaotischen Systemen unterscheiden sich jedoch grundlegend. So fallen Korrelationsfunktionen in gemischten Systemen charakteristischerweise algebraisch ab, im Gegensatz zum exponentiellen Zerfall in voll chaotischen Systemen. Dies liegt darin begründet, dass die chaotische Komponente keine strukturlose Masse ist, sondern von partiellen Transportbarrieren - den Cantori - durchsetzt ist. Diese sind dafür verantwortlich, dass chaotische Trajektorien lange in der Nähe der Inseln hängenbleiben und so der regulären Inseldynamik folgen. Eine prominente Größe, die dieses Hängenbleiben quantifiziert, ist die Wahrscheinlichkeit $P_{\mathrm{cl}}(t)$, dass eine chaotische Trajektorie mindestens eine Zeit $t$ in der Inselhierarchie verweilt [15]. Sie zerfällt gemäß $P_{\mathrm{cl}}(t) \sim t^{-\gamma}$, wobei der Exponent $\gamma>1$ vom speziellen System abhängt. Typische Werte liegen zwischen 1 und 2.5. Die Frage, ob und wie man diesen Exponent direkt aus der Phasenraumstruktur bestimmen kann, ist bis heute ungelöst und wird kontrovers diskutiert.

Quantenchaos, als neue Disziplin, befasst sich mit der Verbindung zwischen klassischer Dynamik und Quantenmechanik. Traditionell gibt es im wesentlichen zwei Stoßrichtungen im Quantenchaos: die Zufallsmatrizentheorie, unterstützt durch die mathematischen Techniken der Supersymmetrie, und semiklassische Methoden. Die Zufallsmatrizentheorie hat ihren Ursprung in der Beschreibung komplizierter Anregungsspektren von Atomkernen und geht auf Arbeiten von Wigner, Dyson und Metha [16] aus den fünfzigern und sechzigern Jahren zurück. Wurde die Theorie der Zufallsmatrizen hauptsächlich zur Beschreibung ungeordneter Systeme herangezogen, so hat die Vermutung von Bohigas, Giannoni und Schmit (1984), dass die statistischen Eigenschaften der Spektren chaotischer Systeme den Vorhersagen der Zufallsmatrizentheorie gehorchen, diesen Zweig neu belebt [17]. Semiklassische Methoden gehen bis auf die Anfänge der Quantenmechanik zurück. Die heuristische Quantisierungbedingung eindimensionaler (integrabler) Systeme von Bohr und Sommerfeld wurde später von Einstein, Brillouin und Keller auf invariante Tori von Systemen mit mehreren Dimensionen übertragen [18-20]. Das Zentrum der Semiklassik chaotischer Systeme ist die Spurformel von Gutzwiller, die eine Verbindung zwischen dem Spektrum eines quantenmechanischen Systems und den periodischen Orbits der klassischen Dynamik herstellt [21-23]. Nicht nur auf geschlossene Systeme wurden die Methoden des Quantenchaos erfolgreich angewandt, sondern auch auf Streusysteme. So konnten universelle Leitwertfluktuationen, die zunächst an ungeordneten Quantenpunkten und später auch an Billards mit chaotischer Dynamik gemessen wurden [24-27], mit Hilfe der Zufallsmatrizentheorie und semiklassischen Methoden erklärt werden [28-31]. In den letzten 15 Jahren lag der Fokus im Quantenchaos nahezu ausschließlich bei der Untersuchung idealisierter vollständig chaotischer Systeme.

Typische Hamilton-Systeme besitzen jedoch einen gemischten Phasenraum und Percival hat vorgeschlagen, die Bereiche chaotischer und regulärer Dynamik unabhängig voneinander zu behandeln [32]. Dieser Idee folgend beschrieben Berry und Robnik die spektralen Eigenschaften gemischter Systeme als Überlagerung eines Poisson- und Zufallsmatrixspektrums [33]. Nach ihrer Auffassung gibt es zwei Typen von Eigenzuständen: Es gibt reguläre 
Eigenfunktionen, die auf den Inseln leben, und chaotische Eigenfunktionen, die die chaotischen Bereiche als Träger haben. Die Vermutung dieser strikten Zweiteilung läuft unter dem Begriff "semiklassische Kondensationshypothese". Eine der wenigen Untersuchungen, die auch die charakteristischen Eigenschaften der Dynamik gemischter Systeme - das algebraische Hängenbleiben $P_{\mathrm{cl}}(t)$ an den Inseln - berücksichtigt, ist die semiklassische Vorhersage fraktaler Leitwertfluktuationen [34]. Sie zeichnen sich dadurch aus, dass der Leitwert als Funktion der Energie oder eines äußeren Magnetfeldes eine fraktale Kurve ist, wobei die fraktale Dimension $D_{0}=2-\gamma / 2$ durch den klassischen Exponenten $\gamma$ bestimmt ist [34]. Dass das Verständnis quantenmechanischer Signaturen gemischter Systeme noch in den Kinderschuhen steckt, zeigen neuere numerische Rechnungen zu Leitwertfluktuationen am Cosinus-Billard [35]. Im Gegensatz zu den vorhergesagten fraktalen Leitwertfluktuationen weist die berechnete Leitwertkurve viele isolierte Resonanzen auf, deren Ursache ungeklärt blieb, und ist sonst überwiegend glatt [35].

In dieser Arbeit werden einige sehr grundlegende Quantensignaturen für Systeme mit gemischtem Phasenraum dargestellt: Das Auftreten einer neuen Klasse von Eigenfunktionen, sowie ihr Einfluss auf die statistischen Eigenschaften von Spektrum und Eigenfunktionen. Der Ursprung der isolierten Resonanzen wird erklärt und die Existenz einer neuen Zeitskala, bis zu der die Quantenmechanik der klassischen Dynamik folgt, wird gezeigt und numerisch untermauert. Weiterhin wird der Dynamik von Wellenpaketen und der Zusammenhang zu spektralen Eigenschaften untersucht.

Der Aufbau dieser Arbeit ist wie folgt: Im zweiten Kapitel werden Grundlagen zu chaotischen Hamilton-Systemen referiert. Kernstück ist das KAM-Theorem, das eine Brücke zwischen integrablen und vollständig chaotischen Systemen herstellt. Ein Modell für den Phasenraumtransport in gemischten Systemen wird vorgestellt. Im dritten Kapitel wird die klassische Dynamik chaotischer Trajektorien in gemischten Systemen untersucht [36]. Es wird gezeigt, dass Renormierungstheorien, die nur eine selbstähnliche Struktur im Phasenraum berücksichtigen, falsche Zerfallsexponenten liefern. Dies gilt selbst dann, wenn der selbstähnliche Bereich die Struktur des Phasenraums dominiert. In den nächsten zwei Kapiteln werden Quantensignaturen des gemischten Phasenraums vorgestellt. Dabei wird sich das Konzept der Flussbarriere als äußerst grundlegend und weitreichend erweisen [37]. Speziell wird im vierten Kapitel eine neue Klasse von Eigenfunktionen eingeführt [38]. Diese hierarchischen Eigenfunktionen leben hinter der Flussbarriere und werden durch ein analytisch zugängliches Modell qualitativ und quantitativ erklärt. Ihr Einfluss auf das Spektrum und die Eigenfunktionsstatistik wird diskutiert. Das fünfte Kapitel vollzieht den Schwenk zu Streueigenschaften gemischter Systeme [39]. Das Hauptaugenmerk liegt dabei auf der Klärung widersprüchlicher Beobachtungen zu Leitwertfluktuationen: fraktale Fluktuationen und isolierte Resonanzen. Es wird gezeigt, dass im Allgemeinen beide Typen von Leitwertfluktuationen gleichzeitig - jedoch auf unterschiedlichen Skalen - vorliegen. Weiterhin wird eine Verbindung zwischen den isolierten Resonanzen im Leitwert und den hierarchischen Zuständen des geschlossenen Systems hergestellt. Diese Ergebnisse liefern eine umfassende Theorie für Leitwertfluktuationen in typischen Hamilton-Systemen. Das 
sechste Kapitel beschäftigt sich mit der Ausbreitung von Wellenpakten und den spektralen Eigenschaften von Bloch-Elektronen im Magnetfeld [40]. Es wird gezeigt, dass ein kürzlich beobachtete Metall-Isolator-Übergang mit Hilfe von vermiedenen Bandkreuzungen verstanden werden kann. Diese führen im Allgemeinen dazu, dass sich das Wellenpaket kreuzförmig ausbreitet. Im siebten Kapitel wird für eine Klasse von Modellen gezeigt, dass die Varianz eines Wellenpakets stärker als ballistisch anwachsen kann [41]. Die Dauer dieser superballistischen Ausbreitung ist verknüpft mit der Zerfallsrate aus der Probe und kann beliebig groß werden. Das achte Kapitel faßt die zentralen Ergebnisse der Arbeit zusammen. 


\section{Kapitel 2}

\section{Hamiltonsches Chaos}

Das Ziel dieses Kapitels ist es, die für diese Arbeit wesentlichen Konzepte zur Beschreibung von Transporteigenschaften niedrigdimensionaler Hamilton-Systeme bereitzustellen. Dabei stehen Hamilton-Systeme mit gemischtem Phasenraum im Zentrum des Interesses. Nach einer kurzen Einführung über dynamische Systeme, werden wichtige Begriffe zur Hamiltonschen Dynamik vorgestellt und am Beispiel gekickter Systeme illustriert. Es wird erklärt, wie die faszinierende Struktur des gemischten Phasenraums entsteht und Transporteigenschaften werden diskutiert. Eine stochastische Beschreibung der Dynamik in der hierarchischen Struktur des Phasenraums liefert ein qualitatives Verständnis gemischter Systeme. Zum Abschluss wird noch auf die quantenmechanische Behandlung von gekickten Systemen eingegangen.

\subsection{Dynamische Systeme}

Viele Phänomene in der Physik lassen sich durch Differentialgleichungen beschreiben. Differentialgleichungen liefern in natürlicher Weise differenzierbare dynamische Systeme [42,43], deren Eigenschaften hier vorgestellt werden.

\subsubsection{Grundbegriffe}

Definition 2.1.1. Unter einem dynamischen System auf einem Gebiet $D$ im $\mathbb{R}^{N}$ versteht man eine differenzierbare Abbildung

$$
\varphi: \Omega \rightarrow D
$$

mit 
1) $\Omega$ ist ein Gebiet im $\mathbb{R} \times D$,

2) $\varphi(0, x)=x$ für alle $x \in D$,

3) $\varphi(t, \varphi(s, x))=\varphi(t+s, x)$.

Das Gebiet $D$ ist der Phasenraum des dynamischen Systems und die Menge aller Punkte $\varphi_{x} \equiv\{\varphi(t, x) \mid t \in \mathbb{R}\}$ heißt Orbit durch den Punkt $x \in D$. Die Orbits bilden eine Zerlegung des Phasenraums, das sogenannte Phasenporträt. Die Abbildung $\varphi(\cdot, x): \mathbb{R} \rightarrow D$ gibt an, wie der Punkt $x$ den Orbit $\varphi_{x}$ durchläuft. Ist die Abbildung injektiv, so kehrt der Punkt $x$ nie zum Ausgangspunkt zurück. Ist die Abbildung hingegen nicht injektiv, so können zwei Fälle auftreten. Entweder $\varphi(t, x)=x$ für alle Zeiten $t$, dann nennt man $x$ einen Fixpunkt des dynamischen Systems, oder es gibt eine minimale Zeit $\rho>0$ mit $\varphi(\rho, x)=x$, und $x$ liegt dann auf einem periodischen Orbit mit der Periode $\rho$. Die Bedingung (3) in Def. (2.1.1) stellt dabei sicher, dass $\rho$ nicht von der Wahl des Punkts $x$ auf dem periodischen Orbits abhängt.

Zwei dynamische Systeme $\varphi$ und $\psi$ sind zueinander konjugiert, wenn es einen Homeomorphismus $h$ und eine stetige, streng monotone Abbildung $\tau$ gibt, so dass Orbits $\varphi_{x}$ auf Orbits $\psi_{x^{\prime}}$ abgebildet werden, d.h.

$$
h\left(\varphi_{t}(x)\right)=\psi_{\tau_{h(x)}(t)}(h(x)) .
$$

Konjugierte dynamische Systeme besitzen demnach das gleiche Phasenporträt. Insbesondere werden Fixpunkte wieder auf Fixpunkte, periodische Orbits wieder auf periodische Orbits abgebildet. Allerdings kann sich die Durchlaufzeit der Orbits verändern.

Im nächsten Abschnitt wird gezeigt, dass jedes dynamische System (zumindest lokal) ein diskretes dynamisches System erzeugt. Dabei besteht ein diskretes dynamisches System aus einem Gebiet $D$ im $\mathbb{R}^{N}$ und einem Diffeomorphismus (bijektive, stetig differenzierbar) $P: D \rightarrow D$. Den Orbit $P_{x}$ von einem Punkt $x$ erhält man dann durch wiederholtes Anwenden von $P$ und der inversen $P^{-1}, P_{x}=\left\{P^{k}(x) \mid k \in \mathbb{Z}\right\}$.

\subsubsection{Poincaré-Abbildung}

Ein nützliches Hilfsmittel zur Untersuchung dynamischer Systeme ist die Poincaré-Abbildung [42]. Sie kann zumindest lokal für periodische Orbits konstruiert werden. Ist $\gamma$ ein periodischer Orbit und $x_{0}$ ein Punkt auf dem Orbit mit Periode $\rho_{0}$, so kann der Geschwindigkeitsvektor $v=d \varphi / d t$ in $x_{0}$ nicht gleich dem Nullvektor sein, da sonst $x_{0}$ ein Fixpunkt wäre. Nun wählt man einen transversalen Schnitt $S$, der in $x_{0}$ senkrecht zu $v$ steht. Liegt der Punkt $x$ auf $S$ hinreichend nah bei $x_{0}$, dann schneidet der Orbit den transversalen Schnitt $S$ wieder zu einem späteren Zeitpunkt. Der Zeitpunkt, wann dies zum ersten Mal passiert, soll mit $\tau(x)$ bezeichnet werden, d.h. die Abbildung $\tau$ ordnet jedem Punkt auf $S$ 


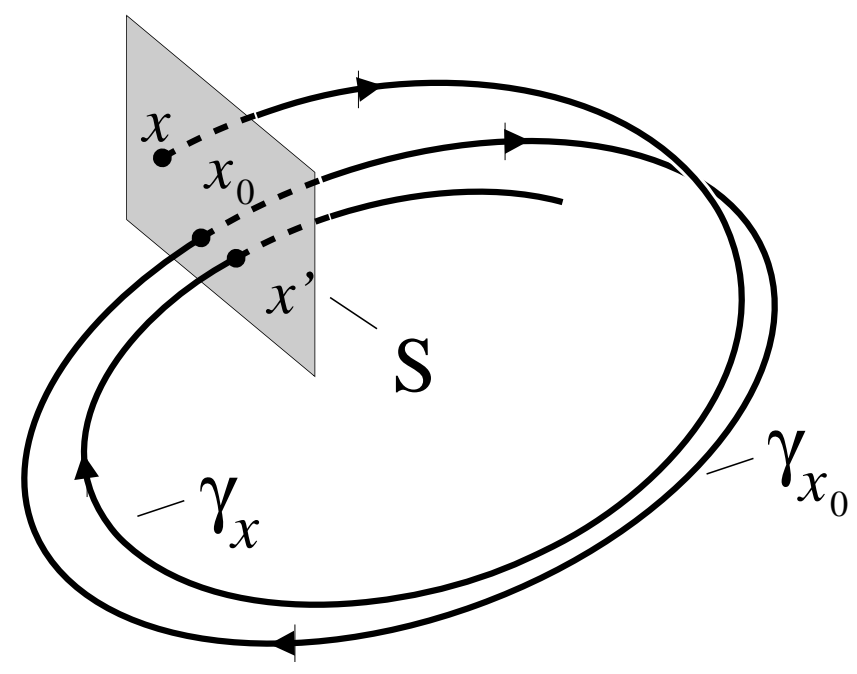

Abbildung 2.1: Ein transversaler Schnitt (Poincaré-Schnitt) $S$ zum periodischen Orbit $\gamma_{x_{0}}$. Der Orbit $\gamma_{x}$ durch den Punkt $x$ schneidet den Poincaré-Schnitt wieder in $x^{\prime}$.

seine Wiederkehrzeit zu. Offensichtlich gilt $\tau\left(x_{0}\right)=\rho_{0}$. Die Poincaré-Abbildung $P$ weist nun jedem Punkt $x$ auf $S$ den nächsten Schnittpunkt $x^{\prime}$ zu

$$
x^{\prime}=P(x) \equiv \varphi(\tau(x), x) .
$$

Dabei wird $x_{0}$ wieder auf $x_{0}$ abgebildet, ist also ein Fixpunkt des dynamischen Systems $P: S \rightarrow S$. Obwohl bei der Konstruktion der Poincaré-Abbildung der genaue Verlauf der Orbits verloren geht, liefert die Untersuchung der Dynamik von $P$ wertvolle Informationen über das Verhalten von $\varphi$. Es bleibt noch anzumerken, dass die Poincaré-Abbildung nicht von der speziellen Wahl des Punkts $x_{0}$ abhängt. Die Poincaré-Abbildung zu einem anderen Punkt $x_{0}^{\prime}$ auf dem selben periodischen Orbit liefert ein dynamisches System $P^{\prime}$, das konjugiert zu $P$ ist und daher die gleichen Informationen enthält.

\section{$2.2 \quad$ Hamiltonsche Dynamik}

\subsubsection{Hamilton-Systeme}

Eine wichtige Klasse dynamischer Systeme bilden die Hamiltonschen Systeme [44]. Sie zeichnen sich dadurch aus, dass die Form der Bewegungsgleichungen invariant unter symplektischen Koordinatentransformationen sind. Dabei heißt ein Diffeomorphismus $h: U \rightarrow$ $\mathbb{R}^{2 N}, U \subset \mathbb{R}^{2 N}$ symplektisch, wenn in jedem Punkt $\boldsymbol{x} \in U$ die Transformation $\mathbf{D} h(\boldsymbol{x})$ die Matrix

$$
\mathbf{S}=\left(\begin{array}{rr}
0 & -\mathbf{I} \\
\mathbf{I} & 0
\end{array}\right)
$$


unverändert lässt, d.h. wenn

$$
[\mathbf{D} h(\boldsymbol{x})]^{T} \cdot \mathbf{S} \cdot \mathbf{D} h(\boldsymbol{x})=\mathbf{S}
$$

für alle $\boldsymbol{x} \in U$ gilt. Hierbei ist $\mathbf{I}$ die Einheitsmatrix in $\mathbb{R}^{N}$. Äquivalent zum Begriff symplektisch wird auch die historische Bezeichnung kanonisch benutzt. In kanonischen Koordinaten lässt sich ein Hamilton-System folgendermaßen definieren.

Definition 2.2.1 (Hamiltonsches System). Gegeben sei ein Gebiet $U$ im $\mathbb{R}^{2 N}$ und eine zweimal stetig differenzierbare Funktion $H: U \rightarrow \mathbb{R}$. Das Gleichungssystem

$$
\dot{q}_{i}=\frac{\partial H}{\partial p_{i}}, \quad \dot{p}_{i}=-\frac{\partial H}{\partial q_{i}}, \quad i=1, \ldots, n
$$

wird als Hamiltonsches System bezeichnet.

Die Funktion $H(\boldsymbol{p}, \boldsymbol{q}, t)$ ist die Hamilton-Funktion des Systems und die Gl. (2.6) nennt man die Hamiltonschen Bewegungsgleichungen. Hängt die Hamilton-Funktion nicht explizit von der Zeit ab, so spricht man von konservativen Hamilton-Systemen. Das Paar $(\boldsymbol{p}, \boldsymbol{q})$ wird als verallgemeinerter Impuls und Ort bezeichnet. Der Zustand des Systems zum Zeitpunkt $t$ wird durch den Vektor von $2 N$ kanonischen Koordinaten $\boldsymbol{x}(t)=(\boldsymbol{p}(t), \boldsymbol{q}(t))$ spezifiziert. Mit dieser Abkürzung lassen sich die Bewegungsgleichungen kompakt schreiben als

$$
\dot{\boldsymbol{x}}=\{\boldsymbol{x}, H\} \quad,
$$

wobei die Poisson-Klammer zwischen zwei Funktionen $f$ und $g$ durch

$$
\{f, g\}=(\nabla f)^{T} \cdot \mathbf{S} \cdot \nabla g
$$

definiert ist. Diese Schreibweise der Bewegungsgleichungen zeigt deutlich die symplektische Struktur Hamiltonscher Systeme.

\subsubsection{Erweiterter Phasenraum}

Für Hamilton-Funktionen, die explizit von der Zeit abhängen, ist es oft nützlich, die Dynamik in einem erweiterten Phasenraum zu betrachten. Die Hamilton-Funktion $\bar{H}$ in diesem erweiterten Phasenraum hat die Form

$$
\bar{H}(\overline{\boldsymbol{p}}, \overline{\boldsymbol{q}})=H(\boldsymbol{p}, \boldsymbol{q}, t)-H t
$$

wobei $(\overline{\boldsymbol{p}}, \overline{\boldsymbol{q}})=(\boldsymbol{p},-H, \boldsymbol{q}, t)$ die neue Koordinaten sind. Der Fluss wird durch eine neue "Zeit" $\xi$ parametrisiert. In den neuen Koordinaten gelten wieder die Hamiltonschen Bewegungsgleichungen und die Hamilton-Funktion hängt nicht mehr explizit von der Zeit $\xi$ ab. Man kann also ein zeitabhängiges Hamilton-System auf ein zeitunabhängiges System abbilden. Dabei nimmt man die um zwei erhöhte Dimension des erweiterten Phasenraums in Kauf. 


\subsubsection{Integralinvarianten}

Eine wichtige Eigenschaft von Hamilton-Systemen ist die Erhaltung des Phasenraumvolumens unter der Dynamik (Satz von Liouville). Für Poincaré-Abbildungen Hamiltonscher Systeme gilt eine ähnliche Eigenschaft. Sei $S$ ein Poincaré-Schnitt im Phasenraum und $A$ eine Fläche auf $S$ mit dem Rand $L$. Die Zeitentwicklung von $L$ liefert einen Zylinder $\Gamma$. Ist nun $L^{\prime}$ ein beliebige andere Schleife auf $\Gamma$, die auf $\Gamma$ homotop zu $L$ ist, so besagt der Satz von Poincaré-Cartan, dass die Wirkungen entlang beider Schleifen gleich sind

$$
\oint_{L} \boldsymbol{p} \mathrm{d} \boldsymbol{q}=\oint_{L^{\prime}} \boldsymbol{p} \mathrm{d} \boldsymbol{q}
$$

Ist $L^{\prime}$ der Rand der Fläche $A^{\prime}$, die aus $A$ durch Anwenden der Poincaré-Abbildung hervorgeht, so folgt, dass beide Flächen gleich groß sind. Die Poincaré-Abbildung Hamiltonscher Systeme ist damit flächenerhaltend.

\subsubsection{Erhaltungsgrößen}

Bei der Untersuchung von Hamiltonschen Systemen spielen Erhaltungsgrößen eine wichtige Rolle. Sie erlauben es, die Dimension des Systems und damit die Komplexität zu reduzieren. Mit Hilfe der Hamiltonschen Bewegungsgleichungen für $\dot{\boldsymbol{p}}$ und $\dot{\boldsymbol{q}}$ lässt sich die totale zeitliche Ableitung einer beliebigen Funktion $\chi=\chi(\boldsymbol{p}, \boldsymbol{q}, t)$ in der Form

$$
\frac{\mathrm{d} \chi}{\mathrm{d} t}=\{\chi, H\}+\frac{\partial \chi}{\partial t}
$$

schreiben. Verschwindet die totale Zeitableitung, so ändert sich $\chi$ entlang einer Trajektorie nicht und ist damit eine Konstante der Bewegung. Funktionen, die nicht explizit von der Zeit abhängen, $\partial \chi / \partial t=0$, sind genau dann Erhaltungsgrößen, wenn sie mit der HamiltonFunktion kommutieren, d.h. die Poisson-Klammer zwischen $\chi$ und $H$ verschwindet. Für konservative Hamilton-Systeme ist die Hamilton-Funktion eine Erhaltungsgröße und gibt die Gesamtenergie des Systems an. Die Dynamik zu einer gegebenen Energie $E$ erfolgt dann auf einer $(2 N-1)$-dimensionalen Untermannigfaltigkeit, die implizit durch die Gleichung $H(\boldsymbol{p}, \boldsymbol{q})=E$ gegeben ist. Existieren noch andere Konstanten der Bewegung, so reduziert sich die Dimension weiter, wenn die Konstanten unabhängig von einander sind und sie in Involution stehen. Die Konstanten $\left\{I_{1}, \ldots, I_{n}\right\}$ sind unabhängig, wenn ihre Gradienten an einem Punkt linear unabhängig sind. Dadurch wird sicher gestellt, dass jede Konstante die Dimension der Untermannigfaltigkeit um eins verringert. Des weiteren stehen zwei Konstanten $I_{i}$ und $I_{j}$ in Involution, wenn ihre Poisson-Klammer verschwindet. Diese Bedingung gewährleistet die Existenz von $n$ kanonischen Variablen $\theta_{i}$, die lokal konjugiert zu den Konstanten $I_{j}$ sind. Es gilt daher $\left\{I_{i}, \theta_{j}\right\}=\delta_{i j}$, was impliziert, dass die Transformation die symplektische Struktur erhält. Gibt es $N$ Konstanten der Bewegung, so ist die Dynamik besonders einfach, wie das folgende Theorem besagt. 
Theorem 2.2.2 (Arnol'd-Liouville). Gibt es $N$ unabhängige Konstanten der Bewegung $\left\{I_{1}, \ldots, I_{N}\right\}$, die in Involution stehen, so erfolgt die Dynamik auf einer Familie $N$-dimensionaler Tori. Es existieren Winkelvariablen $\boldsymbol{\theta}$ und die Bewegung auf den Tori wird durch die Gleichungen

$$
\left(\begin{array}{l}
\dot{\boldsymbol{I}} \\
\dot{\boldsymbol{\theta}}
\end{array}\right)=\left(\begin{array}{c}
0 \\
\boldsymbol{\theta}+\boldsymbol{\alpha}(\boldsymbol{I})
\end{array}\right)
$$

beschrieben.

Besitzt ein Hamilton-System $N$ Konstanten der Bewegung, so heißt es integrabel. Die Gleichungen Gl. (2.12) können direkt integriert werden und es gibt zwei Typen von Orbits: Periodische Orbits liegen genau dann vor, wenn ein Vektor $\boldsymbol{m}$ aus $\mathbb{Z}^{N}$ mit $\boldsymbol{m} \cdot \boldsymbol{\alpha}=0$ existiert. Im Gegensatz zu den periodischen Orbits, deren Maß im Phasenraum verschwindet, besteht für die Windungszahlen typischer Orbits kein rationaler Zusammenhang. Die Dynamik ist dann quasiperiodisch und füllt die Tori vollständig aus.

Eindimensionale konservative Hamilton-Systeme sind aufgrund der Energieerhaltung immer integrabel. Konservative Systeme mit zwei Freiheitsgraden zeigen eine vielfältigere Dynamik. Der Phasenraum hat vier Dimensionen und gibt es - abgesehen von der Energie - keine weitere Erhaltungsgröße, so erfolgt die Dynamik auf einer dreidimensionalen Mannigfaltigkeit. Ein Poincaré-Schnitt reduziert die Dimension weiter und liefert dann eine zweidimensionale Abbildung, die, wie gezeigt wurde, flächenerhaltend ist. Die gleiche Argumentation gilt auch für eindimensionale Hamilton-Systeme, die explizit von der Zeit abhängen. Die Dynamik solcher Systeme wird eingehend im nächsten Abschnitt untersucht.

\subsubsection{Poincaré-Abbildung Hamiltonscher Systeme}

Besonders einfach lässt sich die Poincaré-Abbildung für sogenannte gekickte Systeme berechnen. Sie werden durch eine zeitlich periodische Hamilton-Funktion beschrieben, wobei das Potential nur zu bestimmten Zeiten kurz auf die Dynamik des Teilchens wirkt. Die Hamilton-Funktion eines gekickten Systems hat die Form

$$
H(p, q, t)=f(p)+g(q) \sum_{n \in \mathbb{Z}} \delta(t-n \tau)
$$

wobei $\tau$ der Abstand zwischen zwei Kicks ist. Die Dynamik vollzieht sich auf einer dreidimensionalen Mannigfaltigkeit, parametrisiert durch $(p, q, t)$, und der periodische Antrieb ermöglicht es, die Poincaré-Abbildung als stroboskopische Abbildung für eine Periode zu definieren. Durch Integration der Hamiltonschen Bewegungsgleichungen erhält man so die Abbildung

$$
P(p, q)=\left(\begin{array}{c}
p-g^{\prime}(q) \\
q+\tau f^{\prime}\left(p-g^{\prime}(q)\right)
\end{array}\right)
$$


Aus

$$
\mathbf{D} P(p, q)=\left(\begin{array}{cc}
1 & g^{\prime \prime}(q) \\
\tau f^{\prime \prime}\left(p-g^{\prime}(q)\right) & 1+\tau f^{\prime \prime}\left(p-g^{\prime}(q)\right) g^{\prime \prime}(q)
\end{array}\right)
$$

folgt $\operatorname{Det}(\mathbf{D} P(p, q))=1$, d.h. die Abbildung ist flächenerhaltend.

Es sollen noch zwei prominente Vertreter gekickter Systeme vorgestellt werden, die in dieser Arbeit immer wieder für numerische Untersuchungen herangezogen werden: Zunächst wäre der gekickte Rotator [45] zu nennen, der durch die Impuls- und Potentialfunktion

$$
f(p)=\frac{p^{2}}{2} \quad \text { und } \quad g(q)=K \cos (q)
$$

definiert ist. Mit Gl.(2.14) erhält man die sogenannte Standardabbildung

$$
p_{n+1}=p_{n}+K \sin \left(q_{n}\right) \quad q_{n+1}=q_{n}+\tau p_{n+1},
$$

die erstmals von Boris Chirikov als Modell für die Dynamik von Teilchen in Beschleunigern eingeführt wurde [45]. Da die Potentialfunktion periodisch ist, reicht es aus, die Abbildung auf dem Zylinder $\mathbb{R} \times S^{1}$ zu betrachten. Die spezielle Form der Impulsfunktion sorgt dafür, dass die Standardabbildung auch periodisch in $p$ ist, und erlaubt es weiterhin, die Abbildung auf den Torus $S^{1} \times S^{1}$ zu beschränken. Durch Skalieren von $p_{n}$ sieht man, dass die Eigenschaften der Abbildung nur von $K \tau$ abhängen.

Das gekickte Harper-Modell wird durch

$$
f(p)=K \cos (p) \quad \text { und } \quad g(q)=L \cos (q)
$$

definiert. Es dient als Modell zur Beschreibung von Bloch-Elektronen im Magnetfeld und zeigt eine Vielzahl interessanter Eigenschaften, auf die im Kapitel 6 eingegangen wird. Da $g$ und $f$ periodisch sind, vollzieht sich die Dynamik wie beim gekickten Rotator auf einem Torus.

\subsection{Vom integrablen zum chaotischen Phasenraum}

In diesem Abschnitt werden Hamiltonsche Systeme untersucht, deren Dynamik sich auf einer dreidimensionalen Mannigfaltigkeit abspielt. Das wichtigste Hilfsmittel dabei sind Poincaré-Abbildungen.

\subsubsection{Störung integrabler Systeme}

Im Folgenden soll untersucht werden, wie sich die Dynamik eines integrablen HamiltonSystems unter einer kleinen Störung ändert. Dazu sei $H_{0}(\boldsymbol{I})$ ein zweidimensionales integrables System und $H_{1}(\boldsymbol{I}, \boldsymbol{\theta}, \varepsilon)$ eine Hamiltonsche Störung, die für $\varepsilon=0$ verschwindet. Der 

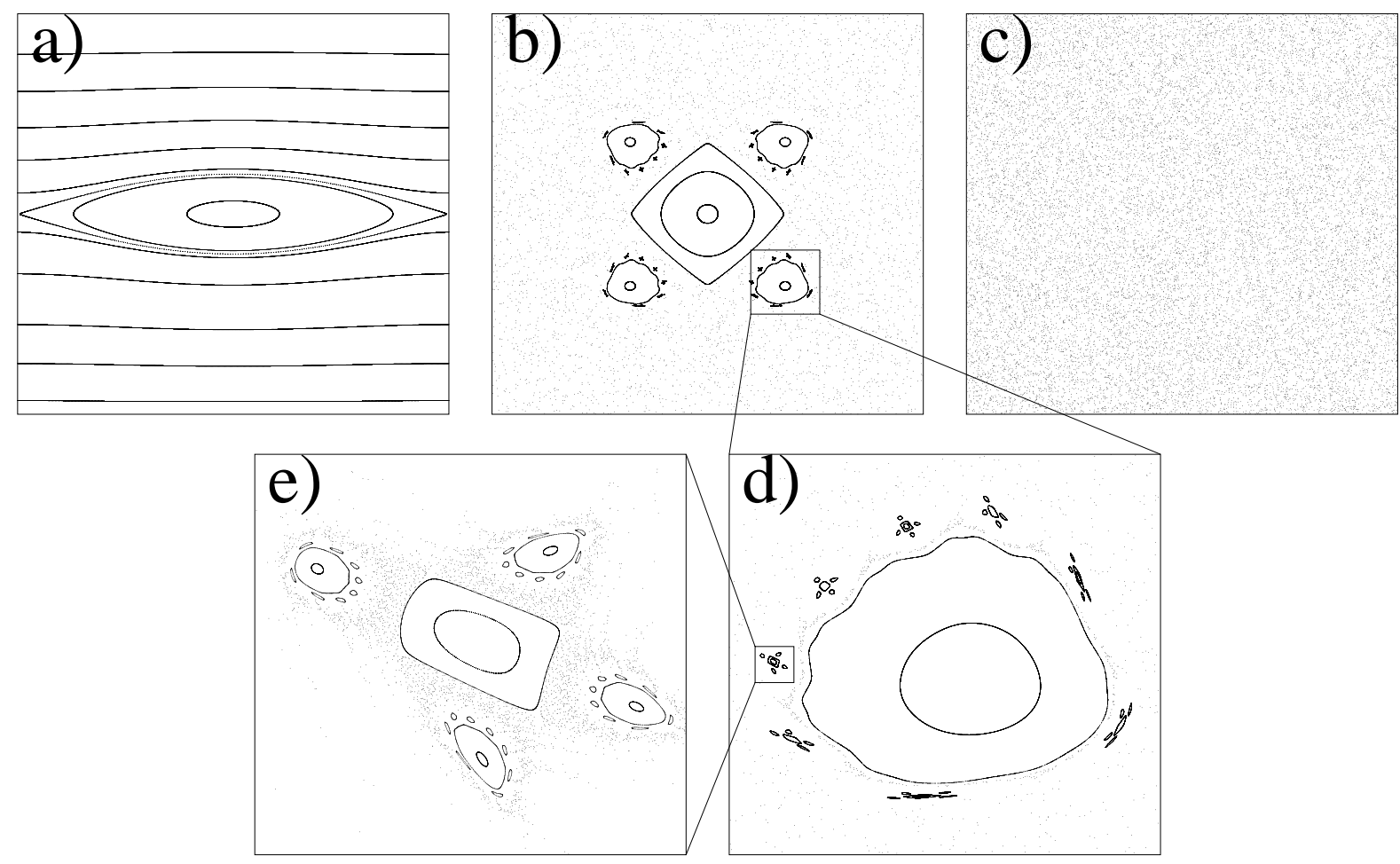

Abbildung 2.2: Phasenraum eines a) nahezu integrablen, b) gemischten und c) vollständig chaotischen Systems. Sukzessive Vergrößerungen des gemischten Phasenraums sind in d) und e) gezeigt.

Poincaré-Schnitt bei $\theta_{2}=$ const liefert die Poincaré-Abbildung

$$
M_{\varepsilon}:\left(\begin{array}{l}
I \\
\theta
\end{array}\right) \rightarrow\left(\begin{array}{ccc}
I & + & f(I, \theta, \varepsilon) \\
\theta+\alpha(I) & + & g(I, \theta, \varepsilon)
\end{array}\right)
$$

wobei $f, g$ periodische Funktionen in $\theta$ sind und $f(I, \theta, 0)=g(I, \theta, 0)=0$ gilt. Da $H_{1}$ eine Hamiltonsche Störung ist, ist auch $H$ eine Hamilton-Funktion und somit $M_{\varepsilon}$ flächenerhaltend für alle $\varepsilon$. Zusätzlich sei noch vorausgesetzt, dass das ungestörte System die TwistBedingung $\mathrm{d} \alpha(I) / \mathrm{d} I \neq 0$ erfüllt. Ist die Abbildung $M_{\varepsilon}$ auf der Kreisscheibe $a \leq I \leq b$ definiert, so liegen alle Windungszahlen $\omega$ zwischen $\alpha(a) / 2 \pi$ und $\alpha(b) / 2 \pi$.

\subsubsection{KAM-Theorie}

Die Dynamik der Poncaré-Abbildung eines zweidimensionalen integrablen Systems ist sehr einfach: Für jedes $I$ ist die Dynamik konjugiert zu einer Rotation auf dem Kreisrand $S^{1}$ (s. Abb. 2.2a). Daher gibt es zwei Arten von Orbits, abhängig davon, ob die Windungszahl $\omega=\alpha(I)$ rational oder irrational ist. Wird die Wirkung $I$ so gewählt, dass $\omega$ rational ist, $\omega=p / q$, dann ist jeder Punkt ein Fixpunkt der $q$-fach iterierten Abbildung. Ist hingegen $\omega$ 
irrational, so gibt es keine periodischen Punkte und die Dynamik ist quasiperiodisch. Wie sich diese beiden Typen invarianter Mengen unter einer kleinen Störung der Abbildung verhalten, soll nun gezeigt werden.

Die Tatsache, dass die gestörte Abbildung auch weiterhin invariante Tori mit irrationaler Windungszahl besitzt, ist der Inhalt der KAM-Theorie, die auf Arbeiten von Kolmogorov, Arnold und Moser beruht [6-10]. Ich möchte hier den Arbeiten von Moser über flächenerhaltende, zweidimensionale Abbildungen folgen, die zu dem folgenden Theorem führen.

Theorem 2.3.1 (Moser Twist Theorem). Die Abbildung $M_{\varepsilon}$ erfülle die Twist-Bedingung und jede geschlossene Kurve $\Gamma$ um a schneide sich mit ihrem Bild $M_{\varepsilon}(\Gamma)$. Ist $\omega$ eine irrationale Zahl zwischen $\alpha(a) / 2 \pi$ und $\alpha(b) / 2 \pi$, die für alle $p, q \in Z$ die Ungleichung

$$
\left|\omega-\frac{p}{q}\right| \leq c|q|^{-5 / 2}
$$

erfülle, dann existiert eine differenzierbare geschlossene Kurve

$$
I=F(\xi, \varepsilon), \quad \theta=\xi+G(\xi, \varepsilon) \quad,
$$

die invariant unter der Abbildung $M_{\varepsilon}$ ist. Die Funktionen $F, G$ sind dabei periodisch in $\xi$. Das Bild eines Punkts $\xi$ auf der Kurve ist gegeben durch $\xi+\omega$.

Ist also $\omega$ hinreichend irrational im Sinne von Gl. (2.20), dann besitzt die gestörte Abbildung $M_{\varepsilon}$ eine invariante Kurve $\gamma$ und die Dynamik auf $\gamma$ ist konjugiert zu einer irrationalen Rotation auf $S^{1}$ mit Windungszahl $\omega$. Die Kurve $\gamma$ wird als KAM-Torus bezeichnet. Der Beweis des Moser Twist Theorem verwendet störungstheoretische Methoden und die Bedingung Gl. (2.20) gewährleistet die Konvergenz der auftretenden Reihen. Man sieht sofort, dass - wenn überhaupt - nur irrationale $\omega$ die Bedingung erfüllen können. Was mit den invarianten Tori mit rationaler Windungszahl geschieht, ist der Inhalt des Theorems von Poincaré-Birkhoff. Es besagt, dass von den überabzählbar vielen periodischen Punkten, die auf einem Torus mit rationaler Windungszahl liegen, nur noch endlich viele überleben.

Theorem 2.3.2 (Poincaré-Birkhoff). Sei $\omega=p / q$ eine rationale Zahl zwischen $\alpha(a) / 2 \pi$ und $\alpha(b) / 2 \pi$, dann besitzt die Abbildung $M_{\varepsilon}^{q}$ genau $2 q$ Fixpunkte, die für hinreichend kleines $\varepsilon$ die Gleichungen

$$
\left(\begin{array}{c}
I_{q} \\
\theta_{q}
\end{array}\right)=\left(\begin{array}{c}
I \\
\theta+2 \pi p
\end{array}\right)
$$

erfüllen.

Die Twist-Bedingung stellt sicher, dass die Gleichung $\theta_{q}=\theta+2 \pi p$ eine eindeutige Lösung $I=F(\theta, \varepsilon)$ mit $F(\theta, 0)=0$ hat. Durch $F$ wird eine Kurve $\gamma$ definiert. Unter $M_{\varepsilon}^{q}$ rotiert $\gamma$ und da $M_{\varepsilon}$ flächenerhaltend ist, müssen sich $\gamma$ und $M_{\varepsilon}^{q}(\gamma)$ schneiden. Es entstehen zwei Arten von Fixpunkten: hyperbolische und elliptische (s. Abb.2.3). In der Nähe eines 

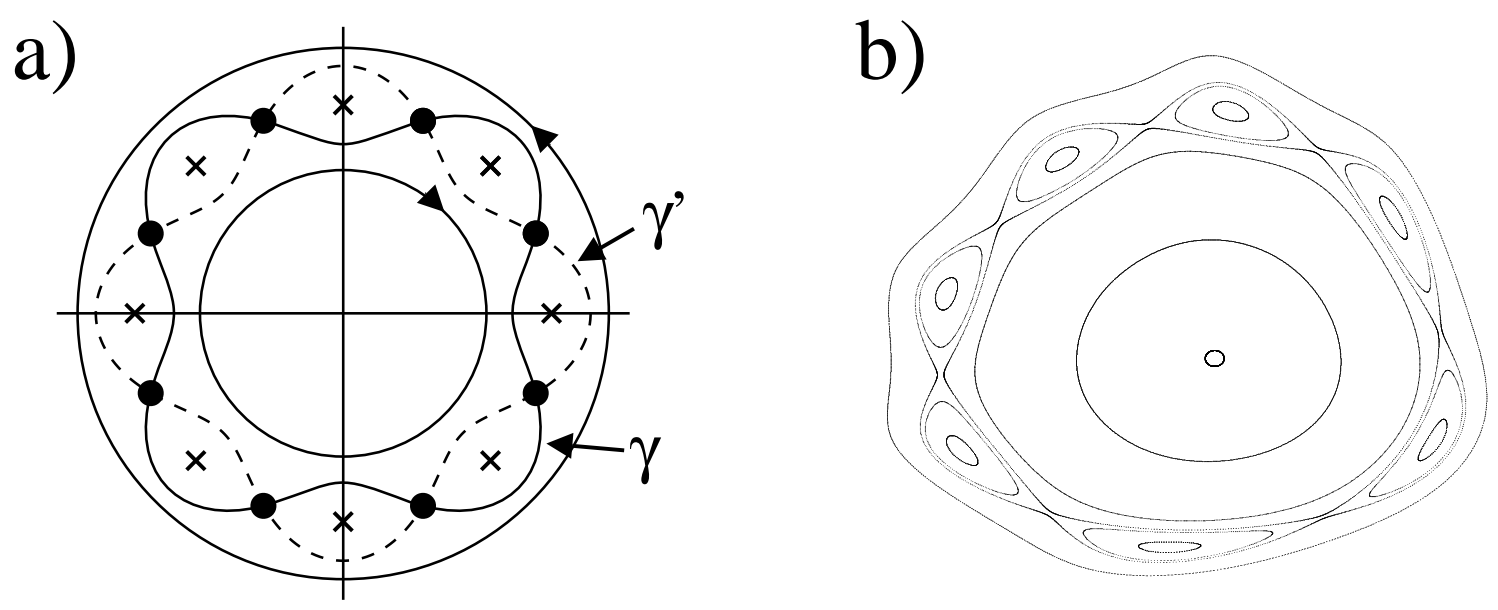

Abbildung 2.3: a)Schematische Darstellung zur Entstehung der Fixpunkte von $M_{\varepsilon}^{q}$. Unter der Dynamik von $M_{\varepsilon}^{q}$ wird die Kurve $\gamma$ auf $\gamma^{\prime}$ abgebildet. Die Punkte markieren die hyperbolischen Fixpunkte, Kreuze kennzeichnen die elliptischen. b) Phasenraumporträt in der Nähe eines elliptischen Fixpunkts. Durch die Störung ist eine Inselkette mit 7 Inseln entstanden.

elliptischen Fixpunkts ist die Dynamik von $M_{\varepsilon}^{q}$ konjugiert zu einer Rotation um den elliptischen Fixpunkt. Es entstehen sogenannte Inseln. Wendet man die obigen beiden Sätze auf die Inseln der Abbildung $M_{\varepsilon}^{q}$ an, so folgt, dass es um diese Inseln wieder eine Kette von Inseln gibt, die nun von einem rationalen Torus von $M_{\varepsilon}^{q}$ stammen. Man erhält so eine unendliche Hierarchie von Inselketten, wie die Vergößerungen in Abb. 2.2 bestätigen. Die hyperbolischen Fixpunkte sind verantwortlich für das Auftreten chaotischer Dynamik, was im nächsten Abschnitt diskutiert wird.

Neben den KAM-Tori gibt es noch eine weitere Klasse von invarianten Mengen mit irrationaler Windungszahl: die Cantori. Sie entstehen aus KAM-Tori, wenn die Störung zu groß wird. Im Gegensatz zu KAM-Tori, die in zwei Dimensionen benachbarte Phasenraumvolumina separieren, weisen Cantori Lücken auf, die einen eingeschränkten Transport erlauben. Ihr Einfluss auf Transporteigenschaften von gemischten Systemen wird im nächsten Abschnitt 2.4 dargestellt. Die bisherigen Ergebnisse dieses Abschnitts sind zusammengefasst im

Theorem 2.3.3 (Aubry-Mather). Sei $M$ eine flächenerhaltende Abbildung des Ringes mit Windungszahlen zwischen a und $b$. Weiterhin erfülle $M$ die Twist-Bedingung. Dann besitzt $M$ für jedes $2 \pi \omega \in[\alpha(a), \beta(b)]$ entweder

1. elliptische und hyperbolische Fixpunkte ( $\omega$ rational) oder

2. einen KAM-Torus ( $\omega$ irrational) oder

3. einen Cantorus ( $\omega$ irrational). 

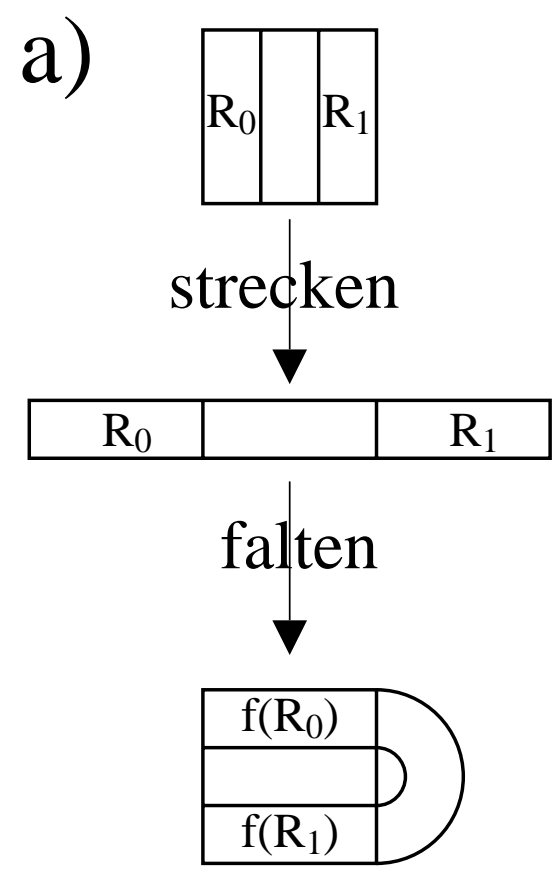

b)

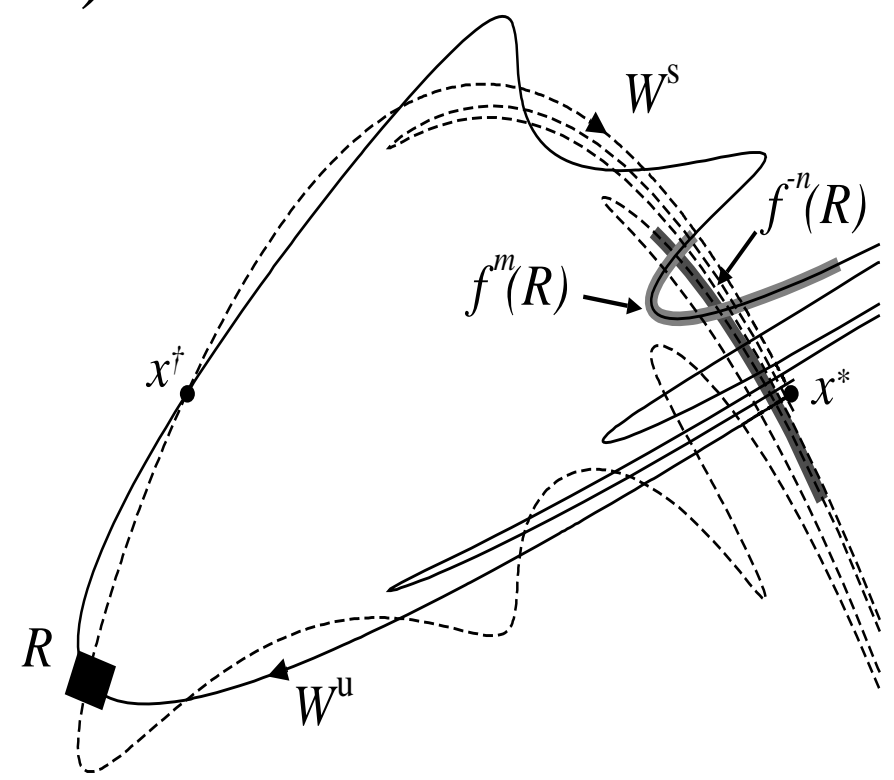

Abbildung 2.4: a) Die Smale Hufeisenabbildung. Zunächst wird das Quadrat entlang der $x$ Richtung gestreckt und in $y$-Richtung gestaucht. Das Rechteck wird dann gefaltet und wieder auf das Quadrat gelegt. b) Die instabile und stabile Mannigfaltigkeit $W^{u}\left(x^{*}\right)$ und $W^{s}\left(x^{*}\right)$ eines hyperbolischen Fixpunkts $x^{*}$ schneiden sich im homoklinen Punkt $x^{\dagger}$. Die Dynamik in der Nähe eines homoklinen Punkts ist konjugiert zur Hufeisenabbildung.

\subsubsection{Chaotische Dynamik}

Neben den regulären Bereichen im gemischten Phasenraum gibt es auch Bereiche chaotischer Dynamik. Zunächst soll an einem einfachen Beispiel - der Hufeisenabbildung - das Auftreten chaotischer Dynamik erklärt werden. Die Hufeisenabbildung besteht aus den folgenden beiden Operationen: Ein Quadrat wird in eine Richtung gestreckt, wobei die Fläche erhalten bleibt. Das so erhaltene Rechteck wird dann gefaltet und wieder auf das Quadrat abgebildet (s. Abb. 2.4). Bei jeder Iteration werden nur ein Teil der Punkte, die in den Bereichen $R_{0}$ und $R_{1}$, wieder auf das Quadrat abgebildet, die anderen Punkte gehen verloren. Es gibt jedoch Punkte, die für immer in dem Rechteck bleiben. Die Menge dieser Punkte sei mit $\Lambda$ bezeichnet, und man sieht leicht, dass diese Punkte eine Cantor-Menge bilden. Eine Cantor-Menge ist dadurch charakterisiert, dass sie keine isolierten Punkte enthält, total unzusammenhängend und kompakt ist. Die Dynamik der Hufeisenabbildung auf $\Lambda$ ist chaotisch im Sinne der

Definition 2.3.4 (Chaotische Dynamik). Sei $f: X \rightarrow X$ ein stetiges diskretes dynamisches System auf dem Raum X. $f$ heißt chaotisch, wenn die beiden folgenden Bedingungen erfüllt sind: 
1. f ist topologisch transitiv.

2. Die Menge der periodischen Orbits von $f$ liegt dicht in $\Lambda$.

Häufig wird noch die Bedingung, dass $f$ sensitiv (positiver Lyapunov-Exponent) von den Anfangsbedingungen abhängt, hinzugenommen. Man kann jedoch zeigen, dass diese Bedingung aus den obigen beiden Bedingungen der Definition folgt. Der Begriff "chaotisch" spiegelt dabei den folgenden Sachverhalt wieder: Charakterisiert $I(t)$ die Infomation, die notwendig ist, ein Segment der Länge $t$ einer Trajektorie zu beschreiben. Für exponentiell instabile Dynamik gilt $[46,47]$

$$
\lim _{t \rightarrow \infty} \frac{I(t)}{|t|}=h_{K S}>0
$$

wobei $h_{K S}$ die Kolmogorov-Sinai Entropie ist. Dieses Resultat zeigt, dass die Information, die man zur Vorhersage eines weiteren Segments einer Trajektorie benötigt, gerade proportional zur Länge des Segments ist. Man kann eine chaotische Trajektorie, auch wenn man sie beliebig lange beobachtet, nicht vorhersagen, wenn man nicht die zu grundliegenden Bewegungsgleichungen kennt.

Der Ursprung der chaotischen Dynamik im gemischten Phasenraum sind die hyperbolischen Fixpunkte. Das Theorem von Hartman-Grobman stellt sicher, dass es in einer Umgebung eines hyperbolischen Fixpunkts $x^{*}$ einer Abbildung $M$ eine lokale stabile und instabile Mannigfaltigkeit gibt. Punkte auf der stabilen Mannigfaltigkeit nähern sich bei jeder Iteration dem Fixpunkt $x^{*}$, Punkte auf der instabilen Mannigfaltigkeit entfernen sich von $x^{*}$. Durch Anwenden von $f$ und der inversen Abbildung $f^{-1}$ auf die lokale stabile und instabile Mannigfaltigkeit erhält man zwei invariante Mengen $W^{s}\left(x^{*}\right)$ und $W^{u}\left(x^{*}\right)$. Schneiden sich $W^{s}\left(x^{*}\right)$ und $W^{u}\left(x^{*}\right)$ in einem weiteren Punkt $x^{\dagger}$, so spricht man von einem homoklinen Schnitt und der Punkt $x^{\dagger}$ heißt homokliner Punkt. Da $W^{s}\left(x^{*}\right)$ und $W^{u}\left(x^{*}\right)$ invariant unter der Dynamik von $M$ sind, müssen die iterierten $M^{k}(q), k \in \mathbb{Z}$ wieder homokline Punkte sein (s. Abb. 2.4). Das Theorem von Smale-Birkhoff stellt nun eine Verbindung zwischen der Hufeisenabbildung und der Dynamik in der Nähe homokliner Punkte her.

Theorem 2.3.5 (Smale-Birkhoff). Ist $f: X \rightarrow X$ ein diskretes dynamisches System. Sei $x^{*}$ ein hyperbolischer Fixpunkt und $x^{\dagger}$ ein homokliner Punkt von $f$. Dann gibt es eine abgeschlossene Menge $\Lambda \subset X$, die $x^{\dagger}$ enthält, mit der Eigenschaft

1. $\Lambda$ ist eine Cantor-Menge.

2. Es gibt ein $p \in \mathbb{Z}$ mit $f^{p}(\Lambda)=\Lambda$.

3. Die Einschränkung von $f^{p}$ auf $\Lambda$ ist topologisch konjugiert zur Hufeisenabbildung (auf der invarianten Menge). 
Die Idee, die dem Beweis zu Grunde liegt, ist in Abb. 2.4b dargestellt. Man betrachtet ein kleines Rechteck $R$ um einen homoklinen Punkt, dessen Seiten parallel zur stabilen und instabilen Mannigfaltigkeit liegen. Die Bilder $f^{n}(R)$ und $f^{-m}(R)$ schneiden sich und die Abbildung $f^{p}$ mit $p=n+m$ verhält sich wie die Hufeisenabbildung.

\subsection{Transport in gemischten Systemen}

Die Diskussion zur chaotischen Dynamik hat gezeigt, dass es nicht sinnvoll ist, das Verhalten von individuellen Trajektorien zu betrachten. Aber gerade die stochastische Eigenschaft der chaotischen Komponenten erlaubt es, mit Hilfe von statistischen Methoden Aussagen über Ensemble von Trajektorien zu machen. Die Dynamik solcher Ensemble wird in der Transporttheorie untersucht. Dabei steht die Frage im Vordergrund, wie lange eine Menge von Trajektorien benötigt, von einer Region im Phasenraum zu einer anderen Region zu gelangen. Dies soll im Folgenden unter "Transport" verstanden werden (exakter wäre der Begriff "Phasenraumtransport"). Ein Verständnis der Transporteigenschaften ermöglicht Vorhersagen zu Übergangswahrscheinlichkeiten und Korrelationsfunktionen.

\subsubsection{Phasenraumfluss}

Eine wichtige Rolle in der Transporttheorie spielt der Phasenraumfluss, der wie folgt definiert ist: Sei $V$ ein Volumen im Phasenraum mit der Oberfläche $\partial V$. Das Volumen der Trajektorien, die das Volumen $V$ pro Zeiteinheit verlassen, ist der Fluss aus $V$. Der NettoFluss, d.h. der Fluss aus $V$ verringert um den Fluss ins Volumen, verschwindet natürlich für Hamiltonsche Systeme. Analog wird auch für diskrete dynamische Systeme der Fluss berechnet. Eine Iteration der Abbildung entspricht dabei einem Zeitschritt. Startet man nun ein Ensemble von $N$ Trajektorien in einem Volumen $V$ und bestimmt die relative Anzahl $P_{\mathrm{cl}}(t)=N(t) / N$ der Trajektorien, die zu einer Zeit $t$ noch im Volumen sind, so findet man einen exponentiellen Zerfall

$$
P_{\mathrm{cl}}(t) \sim e^{-r t}
$$

mit einer Rate $r$, wenn das dynamische System vollständig chaotisch ist. Für ein System mit gemischtem Phasenraum zerfällt diese Verweilzeit jedoch algebraisch [15]

$$
P_{\mathrm{cl}}(t) \sim t^{-\gamma}
$$

Da die mittlere Aufenthaltszeit in einer kompakten Region im Phasenraum endlich sein muss, kommen nur Exponenten mit $\gamma>1$ in Frage [48]. Der Exponent hängt dabei vom speziellen System ab und typische Werte liegen zwischen 1 und 2.5. Warum die Trajektorien im gemischten Phasenraum so lange hängen bleiben, soll nun erklärt werden. 

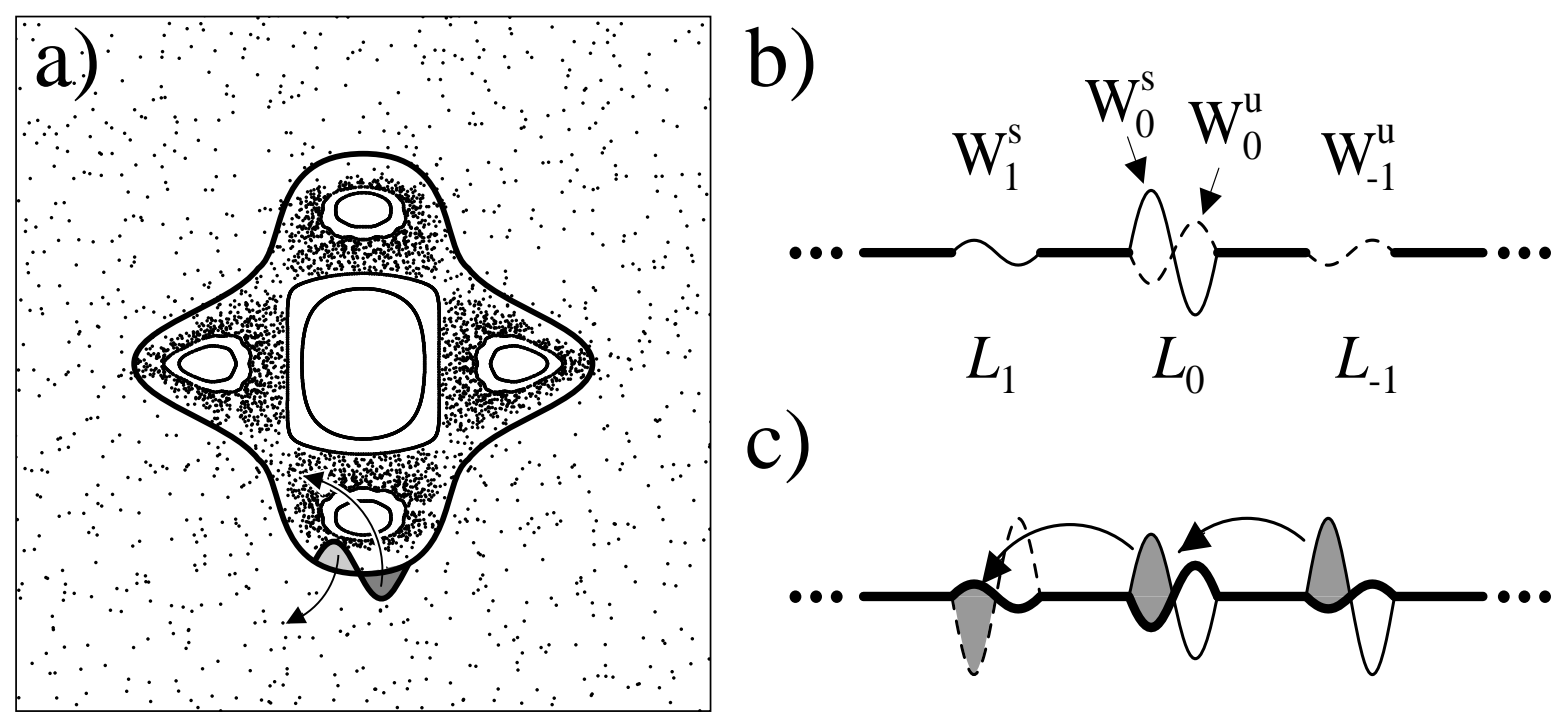

Abbildung 2.5: a) Phasenraum für den gekickten Rotator bei $K=2.5$. Gezeigt ist eine Trajektorie mit Startpunkt in der chaotischen See. Die Trajektorie bleibt lange in der Nähe der Inseln hängen, wie man an der unterschiedlich starken Schwärzung erkennt. Die dicke Linie symbolisiert die partielle Transportbarriere zu einem Cantorus. Der Phasenraumaustausch zwischen dem Bereich innerhalb des Cantorus und der chaotischen See erfolgt durch den turnstile (grau schraffierte Flächen). b) Konstruktion einer partiellen Transportbarriere für einen Cantorus. Die dicken Linienstücke, die durch die Lücken $L_{n}$ unterbrochen werden, bilden den Cantorus. Die Endpunkte der Lücke $L_{0}$ werden durch eine stabile (durchgezogen) und instabile (gestrichelt) Mannigfaltigkeit $W_{0}^{s}$ bzw. $W_{0}^{u}$ verbunden. Die Bilder der stabilen und die Urbilder der instabilen Mannigfaltigkeit bilden mit dem Cantorus die partielle Transportbarriere, die nur durch den turnstile in der Lücke $L_{0}$ passiert werden kann. c) Schematische Darstellung zur Funktion eines turnstile. Der grau schraffierte Phasenraumbereich passiert den Cantorus. Die Pfeile geben die zeitliche Abfolge an.

\subsubsection{Partielle Transportbarrieren}

In Abb. 2.5 ist die Verteilung einer Trajektorie mit Startpunkt in der chaotischen See gezeigt. Im Gegensatz zu einem vollständig chaotischen System ist deutlich zu sehen, dass die chaotische Komponente des Phasenraums für endliche Zeiten nicht gleichmäßig ausgefüllt wird. Die Trajektorie bleibt für viele Iterationen in der Nähe der Inseln hängen. Der Grund hierfür sind partielle Transportbarrieren. Unter einer partiellen Transportbarriere versteht man eine Kurve, die den Phasenraum in zwei Regionen aufteilt und nur einen geringen Phasenraumfluss zwischen den beiden Regionen erlaubt. In einem gemischten Phasenraum kann man zu verschiedenen invarianten Mengen partielle Transportbarrieren konstruieren, z.B. zu periodischen Orbits, homoklinen Orbits oder Cantori. Die Konstruktionsidee ist für die drei Typen die gleiche und soll hier exemplarisch für Cantori dargestellt werden. Ein Cantorus liegt auf einem Lipschitz Graphen und ist homeomorph zu einem Kreisring, aus dem eine abzählbare Menge von offenen Intervallen herausgenommen wurde. Die End- 
punkte dieser Lücken gehören dabei zum Cantorus. Seien nun $x_{0}$ und $y_{0}$ die Endpunkte zu einer Lücke $L_{0}$ im Cantorus. Da der Cantorus eine invariante Menge bildet und die Dynamik auf dem Cantorus monoton ist, sind die Iterierten $x_{t}$ und $y_{t}$ wieder Endpunkte von Lücken im Cantorus. Da die Länge des Cantorus endlich ist, muss der Abstand zwischen den Endpunkten hinreichend schnell verschwinden. Die Menge aller Lücken, die durch Iteration aus einer Lücke hervorgehen, nennt man eine Familie. Ein Cantorus kann maximal abzählbar viele solcher Familien enthalten. Um die Darstellung zu erleichtern, wird im Folgenden davon ausgegangen, dass es nur eine Familie von Lücken gibt, d.h. man erhält jede Lücke durch Iteration von $L_{0}$. Zu jeder Lücke $L_{t}$ im Cantorus gibt es eine stabile Mannigfaltigkeit $W_{t}^{s}$ und eine instabile $W_{t}^{u}$, die die Endpunkte der Lücke verbinden. Eine partielle Transportbarriere entsteht, indem man den Cantorus um die Kurvenstücke $W_{t}^{u}$ für $t \leq 0$ und $W_{t}^{s}$ für $t>0$ ergänzt (s. Abb. 2.5b). Das Urbild von $W_{1}^{s}$ liegt in der Lücke $L_{0}$. Wäre es identisch mit $W_{0}^{u}$, so hätte man eine geschlossene invariante Menge konstruiert, einen KAM-Torus und keinen Cantorus. Daher müssen $W_{1}^{s}$ und $W_{0}^{u}$ zwei verschiedene Kurven sein, die sich mindestens in einem Punkt schneiden, da beide durch der Minimax-Orbit gehen, welcher zwischen den beiden Endpunkten liegt. $W_{1}^{s}$ und $W_{0}^{u}$ bilden eine Schleife in Form einer 8. Eine solche Schleife nennt man turnstile, da dessen Dynamik einer Drehtür gleicht (s. Abb. 2.5c). Um den Cantorus zu passieren, muss ein Orbit durch den turnstile. Die Fläche $\Delta W$ des turnstile gibt daher den Phasenraumfluss durch den Cantorus an.

Da der Flächeninhalt des turnstile nur schwer bestimmbar ist, verfährt man in der Praxis folgendermaßen: $\mathrm{Zu}$ einem Cantorus mit Windungszahl $\omega$ wählt man eine Folge von $\left(m_{k}, n_{k}\right)$ periodischen minimalisierenden Orbits $\left(x^{k}\right)$ und Minimax-Orbits $\left(y^{k}\right)$, deren Windungszahl $m_{k} / n_{k}$ gegen $\omega$ konvergiert. Die Punkte $x^{k}$ und $y^{k}$ sind dabei Repräsentanten des Orbits. Berechnet man nun zu jedem $k$ die Wirkungen für die beiden Orbits $S\left(x^{k}\right)$ und $S\left(y^{k}\right)$, so konnte Mather zeigen, dass der Grenzwert

$$
\Delta W_{\omega}=\lim _{k \rightarrow \infty}\left[S\left(y^{k}\right)-S\left(x^{k}\right)\right]
$$

existiert und nicht negativ ist. Ist $\Delta W_{\omega}>0$, so gibt es keinen invarianten Kreis mit Windungszahl $\omega[49] . \Delta W_{\omega}$ ist dann gleich dem Fluss durch den Cantorus, also gleich der Fläche des turnstile [50].

Warum gerade die Cantori eine wichtige Klasse von partiellen Transportbarrieren bilden, hat den folgenden Grund. Das KAM-Theorem stellt sicher, dass es in einer Umgebung eines elliptischen Fixpunkts invariante KAM-Tori gibt. Diese bilden die Insel. Bei gegebener Störstärke gibt es einen äußeren KAM-Torus, der den Rand der Insel markiert. Innerhalb dieses Torus liegen weitere KAM-Tori, außerhalb existieren jedoch keine KAM-Tori. Wird nun die Störstärke erhöht, so zerbricht der Torus und es entsteht ein Cantorus. Dabei ändert sich der Fluss durch den Torus stetig [13]. Da der Fluss durch KAM-Tori verschwindet, gibt es in der Nähe der Inseln Cantori mit beliebig kleinen Flüssen. Diese können Trajektorien lange in der Nähe der Inseln festhalten. Wird die Störstärke weiter erhöht, so werden die Lücken im Cantorus immer größer und der Einfluß des Cantorus auf die Dynamik nimmt ab. 


\subsubsection{Markov-Modell zum Phasenraumtransport}

In diesem Abschnitt wird ein Markov-Modell zur Beschreibung des Transports in gemischten Systemen vorgestellt. Es geht auf Arbeiten von MacKay, Meiss, Ott und Percival [50-53] zurück, die den Transport zwischen Regionen im Phasenraum untersucht haben, die durch partielle Transportbarrieren wie Cantori oder stabile und instabile Mannigfaltigkeiten getrennt sind.

Das Markov Modell beruht auf einer Partitionierung der chaotischen Komponente des Phasenraums in Regionen $R_{s}$ mit Volumina $\Omega_{s}$. Die Dynamik in jeder Region wird als stochastisch angenommen und der Phasenraumaustausch zwischen benachbarten Regionen $R_{s}$ und $R_{r}$ ist durch den Fluss $\Phi_{s}$ gegeben. Die Partionierung des Phasenraums ist jedoch nicht trivial. Insbesondere sollten solche partiellen Transportbarrieren einen starken Einfluß auf die Dynamik haben, die nur einen kleinen Fluss zwischen den benachbarten Regionen erlauben. MacKay et al. [52] haben vorgeschlagen, Cantori für die Partitionierung des Phasenraums zu verwenden, da der Fluss durch Cantori in der Nähe der Inseln stetig abnimmt. (Später wurden auch partielle Transportbarrieren aus stabilen und instabilen Mannigfaltigkeiten untersucht [54].)

In Abb. 2.6 ist eine typische Partitionierung des Phasenraums in der Nähe einer Hauptinsel mit drei Nebeninseln, die wiederum von einer Inselkette umgeben sind, dargestellt. Das Markov-Modell hat dann die Form eines binären Baums und jeder Zustand $s$ wird eindeutig durch eine endliche Folge von Symbolen "R" und "L" beschrieben. Auf jeder Stufe $s$ der Hierarchie gibt es für eine Trajektorie zwei Möglichkeiten tiefer in die Hierarchie vorzudringen: Entweder die Trajektorie nähert sich weiter der zentralen Insel der Hierarchiestufe, in diesem Fall wird dem Index eine "L" angehängt, oder sie springt in die umgebende Inselkette und dem Index wird eine "R" hinzugefügt. Der Ast, der nur das Symbol "L" enthält, beschreibt Trajektorien, die sich ausschließlich der Hauptinsel nähern. Das andere Extrem $s=$ LR $\cdots$ entspricht Trajektorien, die sich auf jeder Hierarchiestufe der Inselkette nähern, die die zentrale Insel der Stufe umgibt.

Die Dynamik in jeder Region wird als vollständig chaotisch angenommen. Für jede Region $R_{s}$ gibt es zwei wichtige Zeitskalen: Die Mixing-Zeit ist die Zeit, die ein Ensemble von Trajektorien benötigt, gleichmäßig die Region auszufüllen. Hiermit konkurriert die mittlere Zeit, die eine Trajektorie in dieser Region verweilt. Diese Zeit ist gegeben durch das Verhältnis des Volumens $\Omega_{s}$ zur Fläche des turnstile, also dem Fluss $\Phi_{s}$. Im Rahmen des Markov-Modells wird angenommen, dass für jede Stufe $s$ die Mixing-Zeit klein gegenüber der mittleren Verweilzeit in der Region ist. Sind die Volumina $\Omega_{s}$ und die Flüsse $\Phi_{s}$ bekannt, so ist das Markov-Modell eindeutig bestimmt und die Dynamik auf dem Baum kann untersucht werden. Dabei ist man insbesondere an der Verteilung der Verweilzeiten für Trajektorien interessiert, die in der Region $R_{0}$ - der chaotischen See - starten, einen Teil des Baums erkunden und schließlich wieder zum Ausgangspunkt zurückkehren. Ohne weitere Annahmen über die Volumina $\Omega_{s}$ und die Flüsse $\Phi_{s}$ kann die Verteilung nur numerisch 

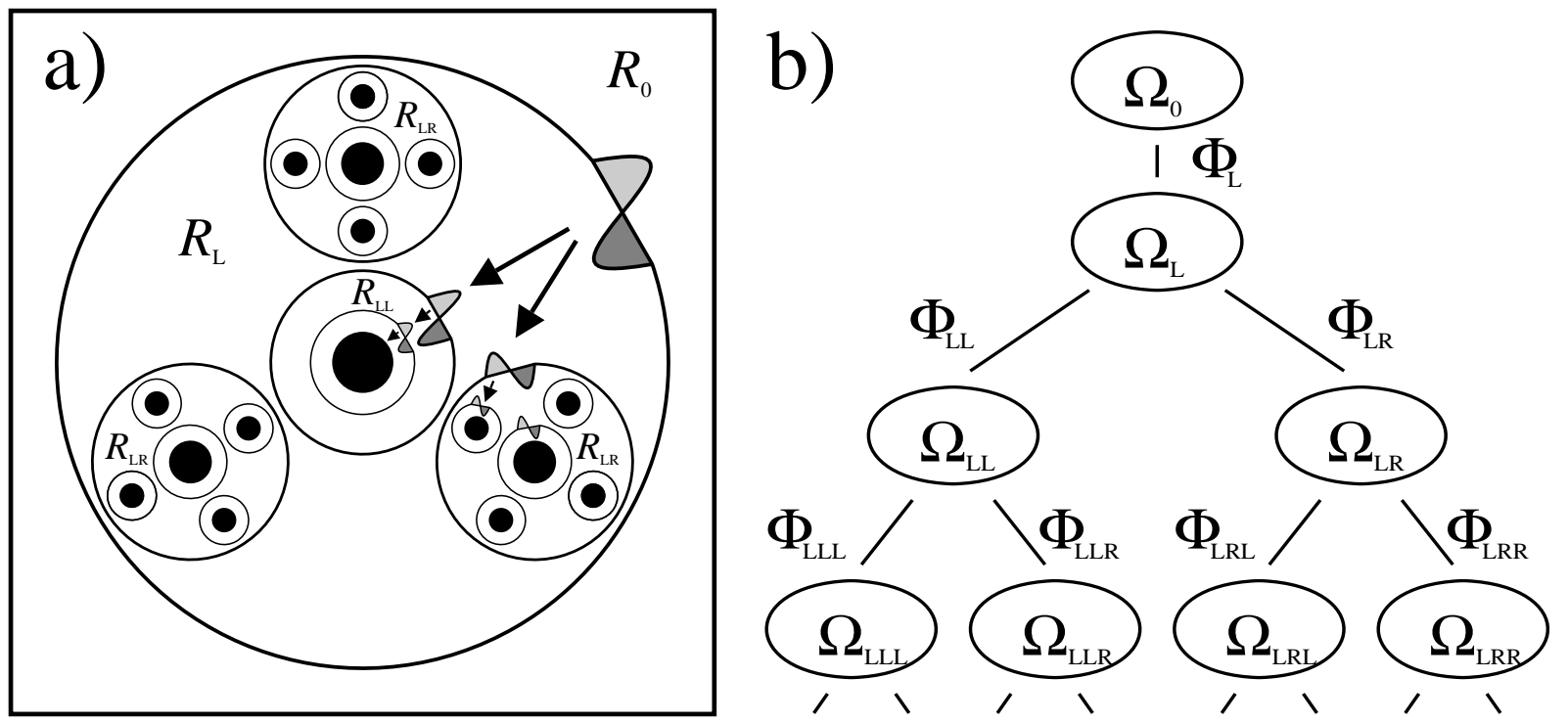

Abbildung 2.6: a) Schematische Darstellung der Partitionierung des Phasenraums durch Cantori. In der Mitte liegt die Hauptinsel umgeben von einer Inselkette, die vier Nebeninseln besitzt. Die Kreise symbolisieren die Cantori, die Schleifen die turnstiles. Zwei extreme Pfade in dieser Cantori-Hierarchie sind durch die Pfeile angegeben: Die obere Pfeilfolge beschreibt Trajektorien, die sich ausschließlich der Hauptinsel nähern. Das Hängenbleiben in den Insel-um-Insel Strukturen wird durch die untere Pfeilfolge wiedergegeben. b) Das Markov-Modell zur Beschreibung der Dynamik im Phasenraum ist ein binärer Baum. Abgesehen von der Möglichkeit wieder zur nächst-höheren Stufe zurückzukehren, hat eine Trajektorie in jeder Stufe zwei Möglichkeiten: Entweder sie nähert sich weiter der Hauptinsel der Stufe auf der sie sich befindet, oder sie bleibt an der nächsten Inselkette hängen.

berechnet werden.

Ein qualitatives Verständnis der Dynamik erlaubt das Kettenmodell. Im Gegensatz zum Baum wird im Kettenmodell nur ein Ast betrachtet, z.B. der Ast des Baums, der das Hängenbleiben von Trajektorien, die sich ausschließlich der Hauptinsel nähern, beschreibt. Das Kettenmodell besteht also aus Regionen $n \in \mathbb{N}$ mit Volumina $\Omega_{n}$, die durch Flüsse $\Phi_{n, n+1}$ verbunden sind. Weiterhin sei angenommen, dass die Volumina und Flüsse gemäß

$$
\Omega_{n}=\Omega_{0} \omega^{n} \quad \text { und } \quad \Phi_{n, n+1}=\Phi_{0} \varphi^{n}
$$

skalieren, wobei $0<\varphi<\omega<1$ gelten soll. Für dieses Modell haben Hanson, Cary und Meiss [53] gezeigt, dass die Wahrscheinlichkeit $P_{\mathrm{cl}}(t)$ länger als eine Zeit $t$ in der Kette hängenzubleiben algebraisch abfällt

$$
P_{\mathrm{cl}}(t) \sim t^{-\gamma} \quad \text { mit } \quad \gamma=\left(1-\frac{\ln \omega}{\ln \varphi}\right)^{-1} .
$$


Der Exponent $\gamma$ wird dabei durch die Skalierungsparameter bestimmt. Der Beweis dieser Aussage ist jedoch sehr technisch und umfangreich, weshalb er hier nicht dargestellt werden soll [53]. Anschaulich gelang man folgendermaßen zu Gl. (2.28): Jede Region für sich genommen erzeugt einen exponentiellen Zerfall mit der Rate $\Phi_{n, n+1} / \Omega_{n}$. Wie stark die $n$-te Region an die Region $R_{0}$ koppelt, ist durch den Fluss $\Phi_{n, n+1} \sim \varphi^{n}$ gegeben. Die Verteilung $P_{\mathrm{cl}}(t)$ erhält man nun als Überlagerung der exponentiellen Zerfälle mit den Raten $\sim(\varphi / \omega)^{n}$. Wie wichtig der Beitrag des Zerfalls der $n$-ten Region für die Verteilung ist, wird durch den Fluss $\sim \varphi^{n}$ bestimmt. In einer doppelt-logarithmischen Darstellung hat die Verteilung die mittlere Steigung $\ln (\varphi) / \ln (\omega \varphi)$. Dies liefert den Zusammenhang in Gl. (2.28). Auf Grund der Einfachheit des Kettenmodells wird dies bei der quantenmechanischen Untersuchung des Phasenraums noch eine wichtige Rolle spielen.

\subsection{Quantenmechanik gekickter Systeme}

Zum Abschluss dieses Kapitels soll noch auf die quantenmechanische Beschreibung von gekickten Systemen eingegangen werden. Grundlagen zum Quantenchaos finden sich z.B. in den Büchern von Haake [55] oder Stöckmann [56]. Kanonische Quantisierung der HamiltonFunktion in Gl. (2.13), d.h. Ersetzen der reellen Variablen $p$ und $q$ durch Operatoren $\hat{p}$ und $\hat{q}$, die der Vertauschungsrelation $[\hat{q}, \hat{p}]=\imath \hbar$ genügen, führt auf das zugehörige gekickte Quantensystem. Der klassischen Poincaré-Abbildung entspricht der unitäre Zeitentwicklungsoperator $U$, der einen Zustand $\psi(t)$ in den Zustand $\psi(t+\tau)$ überführt. Der Zeitentwicklungsoperator für eine Periode lässt sich leicht ausrechnen

$$
U=e^{-\frac{2}{\hbar} \int_{0}^{\tau} H(\hat{p}, \hat{q}, t) \mathrm{d} t}=e^{-\frac{2}{\hbar} \tau f(\hat{p})} e^{-\frac{2}{\hbar} g(\hat{q})} .
$$

\section{Berechnung des Spektrums}

Da das Potential $g$ als $2 \pi$-periodisch angenommen wird, haben die Eigenfunktionen $\phi$ des Zeitentwicklungsoperators $U$ nach Blochs Theorem in der Ortsdarstellung die Form $\phi(q)=e^{\imath q \theta_{q}} \phi_{\theta_{q}}(q)$ mit $\phi_{\theta_{q}}(q+2 \pi)=\phi_{\theta_{q}}(q)$. Dies hat zur Konsequenz, dass die Eigenwerte des Impulsoperators diskret sind. Sie sind gegeben durch $\hbar\left[n+\theta_{q} /(2 \pi)\right]$, wobei $n \in \mathbb{Z}$ gilt. Im Allgemeinen ist $U$ eine unendlich dimensionale Matrix. Ist jedoch das Impulsgitter invariant unter der Transformation $n \rightarrow n+M /(2 \pi \hbar)$, dann gibt es eine ganze Zahl $N$ mit $\hbar=2 \pi M / N$ und $U$ ist ebenfalls translationsinvariant. Dadurch reduziert sich die Dimension von $U$ auf $N$. Die Periodizität des in Impulsrichtung ausgedehnten Systems wird durch eine weitere Bloch-Phase $\theta_{p}$ berücksichtigt. Zu gegebenen Phasen $\theta_{p}, \theta_{q}$ kann man die Matrix $U$ diagonalisieren und erhält $N$ Eigenwerte. Da die Matrix $U$ unitär ist, muss jeder Eigenwert von der Form $e^{\imath \omega}$ sein, wobei $\omega \in \mathbb{R}$ als Quasi-Energie bezeichnet wird. Das Spektrum der Quasi-Energien der Matrix $U$ erhält man als Vereinigung der Teilspektren von $U\left(\theta_{p}, \theta_{q}\right)$ zu allen Bloch-Phasen $\theta_{p}, \theta_{q} \in[0,2 \pi]$ [57]. 


\section{Zeitentwicklung}

Ist man an der Zeitentwicklung eines Wellenpakets interessiert, so verwendet man zweckmäßigerweise Fourier-Transformationen, um zwischen den Darstellungen des Wellenpakets im Orts- und Impulsraum zu wechseln. So kann ausgenutzt werden, dass die einzelnen Operatoren aus denen sich $U$ zusammensetzt, in jeweils einer der beiden Darstellungen diagonal sind. Der Rechenaufwand für jede Iteration beträgt demnach $\sim N \ln N$, wobei $N$ die Anzahl der Zustände angibt. Will man die Dynamik im offenen Quantensystem studieren, so werden nach jeder Anwendung von $U$ Beiträge des Wellenpaket außerhalb eines Fensters, z.B. außerhalb der ersten Einheitszelle, gelöscht. Mathematisch wird dies durch einen Projektionsoperator $P$ beschrieben, der das Wellenpaket auf den gewünschten Unterraum projiziert. Der Zeitentwicklungsoperator ist dadurch nicht mehr unitär und die Norm des Wellenpakets zerfällt mit jeder Iteration.

\section{Phasenraumdarstellung}

Schwerpunkt dieser Arbeit ist das Zusammenspiel von quantenmechanischen und klassischen Eigenschaften. Obwohl es in der Quantenmechanik nicht das Konzept des Phasenraums gibt, existieren Verfahren, die es erlauben, Wellenfunktionen $\phi$ auf den Phasenraum zu projizieren. Zunächst wäre da die Wigner-Funktion zu nennen [58]. Sie hat jedoch den Nachteil, dass sie Oszillationen aufweist, die zu negativen Werten führen können. Gebräuchlicher ist daher die Husimi-Darstellung

$$
<q, p\left|\phi>=\left(\frac{s}{\pi \hbar}\right)^{1 / 4} \int \exp \left(-\frac{s(x-q)^{2}}{2 \hbar}-\imath \frac{p(x-q / 2)}{\hbar}\right)<x\right| \phi>d x .
$$

Hierbei nehmen $p$ und $q$ die klassischen Werte im Phasenraum an. Der Parameter $s$ beschreibt die Asymmetrie der Gauß-Glocke in $p$ und $q$ Richtung. 


\section{Kapitel 3}

\section{Chirikovs siebtes Gesetz}

Im letzten Kapitel wurde gezeigt, dass Korrelationen in typischen Hamilton-Systemen algebraisch abfallen. Dies gilt insbesondere für die Verteilung der Aufenthaltszeiten $P_{\mathrm{cl}}(t) \sim t^{-\gamma}$ chaotischer Trajektorien in der Nähe der regulären Dynamik. Der Exponent $\gamma$, bestimmt durch numerische Simulationen, scheint hingegen nicht universell zu sein, sondern hängt vom betrachteten System und von Parametern ab. Er liegt typischerweise zwischen 1 und 2.5. Es ist eine fundamentale Frage im Gebiet Hamiltonscher Dynamik, ob asymptotisch ein universeller Exponent existiert.

Basierend auf der universellen Existenz von kritischen goldenen Tori, haben Chirikov und Shepelyansky argumentiert, dass die Aufenthaltswahrscheinlichkeit unabhängig vom System und von Parametern asymptotisch wie $P_{\mathrm{cl}}(t) \sim t^{-3}$ zerfällt [59] (bekannt als Chirikovs siebtes Gesetz [60]). Ihre Argumente beruhen auf Untersuchungen zum gekickten Rotator zur Kickstärke $K=K_{c}$, bei der der goldene Torus gerade kritisch ist. Im Gegensatz hierzu liefern Untersuchungen zum Hängenbleiben von Trajektorien in Insel-um-Insel Strukturen typischerweise kleinere Exponenten. So haben Zaslavsky et. al. gefunden, dass bei der Kickstärke $K=K^{*}$ der gekickte Rotator eine ausgeprägte Insel-um-Insel Struktur besitzt, die sich dadurch auszeichnet, dass jede Insel immer von genau acht Tochterinseln umgeben ist [61]. Unter Ausnutzung diese exakten Skalierung haben sie den Exponenten $\gamma=2.25$ für das Hängenbleiben berechnet. Um diese Widersprüche zu aufzuklären, wird im Folgenden der gekickte Rotator zu den beiden Kickstärken $K_{c}$ und $K^{*}$ genauer numerisch untersucht [36].

\subsection{Kritischer goldener Torus}

Die KAM-Theorie besagt, dass hinreichend irrationale Tori unter einer kleinen Störung erhalten bleiben. Wie anfällig ein Torus auf die Störung reagiert, hängt von der Ket- 


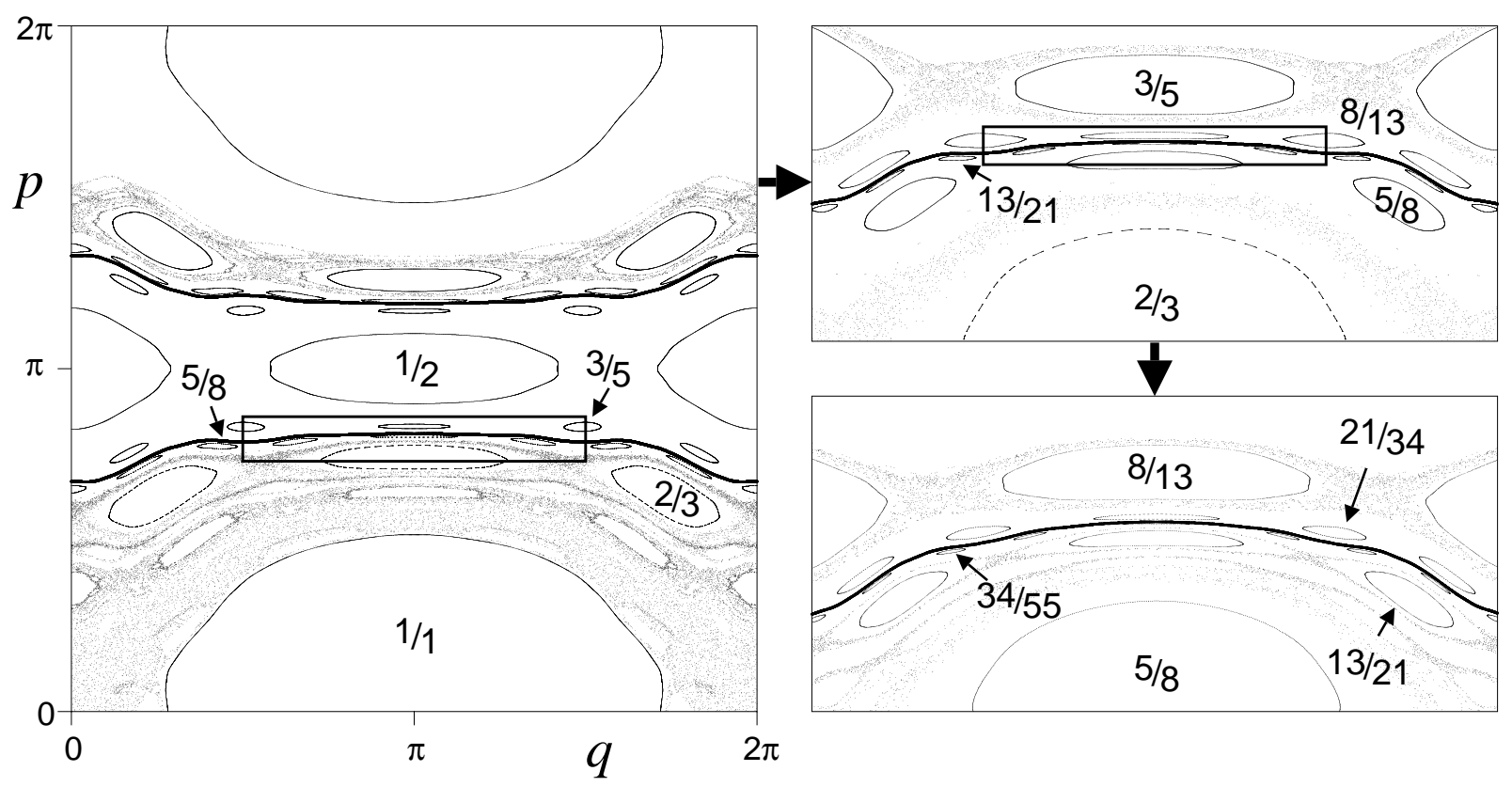

Abbildung 3.1: Phasenraum für den gekickten Rotator bei $K=K_{c}$ in symmetrischer Darstellung. Bei diesem Parameter ist der goldene Torus (dicke Linie) gerade kritisch. Die Vergrößerungen zeigen die selbstähnliche Struktur des Phasenraums in der Nähe des goldenen Torus. Die Zahlen geben die Windungszahlen $p_{n} / q_{n}$ der Inselnketten an, die den goldenen Torus approximieren.

tenbruchentwicklung seiner Windungszahl $\omega$ ab. Ein Torus ist um so stabiler, je kleiner die Beträge der Zahlen in der Kettenbruchentwicklung sind. Am stabilsten erweisen sich daher die sogenannten noblen Tori, die sich durch Windungszahlen mit Kettenbruchentwicklungen $\omega=\left[a_{1}, \ldots, a_{n}, 1,1,1 \ldots\right]$ auszeichnen (Greensche Vermutung [62]). Die Phasenraumstruktur in der Nähe eines kritischen noblen Torus ist unabhängig von der betrachteten Abbildung und in diesem Sinne universell [63]. Dabei heißt ein Torus kritisch, wenn die Störstärke gerade so gewählt ist, dass eine beliebig kleine Erhöhung den Torus zerstört [62,63]. Auf Grund ihrer Stabilität und universellen Struktur wurden kritische noble Tori intensiv untersucht [15,45,62-64], dies gilt insbesondere für goldene Tori mit $\omega_{c}=(\sqrt{5}-1) / 2=[1,1,1, \ldots]$. In Abb. 3.1 ist der Phasenraum für den gekickten Rotator mit $K=K_{c}=0.97163540631$ dargestellt. Bei dieser Kickstärke ist der goldene Torus $p_{c}$ gerade kritisch [65]. Der kritische goldene Torus wird durch Inselketten, den sogenannten prinzipiellen Resonanzen, mit den Windungszahlen $p_{n} / q_{n}$ approximiert, wobei $p_{n}$ und $q_{n}$ zwei aufeinander folgende Fibonacci-Zahlen sind. Deutlich erkennbar ist die selbstähnliche Struktur des Phasenraums in der Nähe von $p_{c}$.

Unter der Annahme, dass das Hängenbleiben chaotischer Trajektorien von den prinzipiellen Resonanzen dominiert wird, kann mit Hilfe renormierungstheoretischer Argumente die Vorhersage $\gamma=3$ für den asymptotischen Zerfall von $P_{\mathrm{cl}}(t)$ abgeleitet werden [53,63,66]. Im Gegensatz zu dieser Vorhersage zeigen numerische Untersuchungen einen deutlich schwächeren 

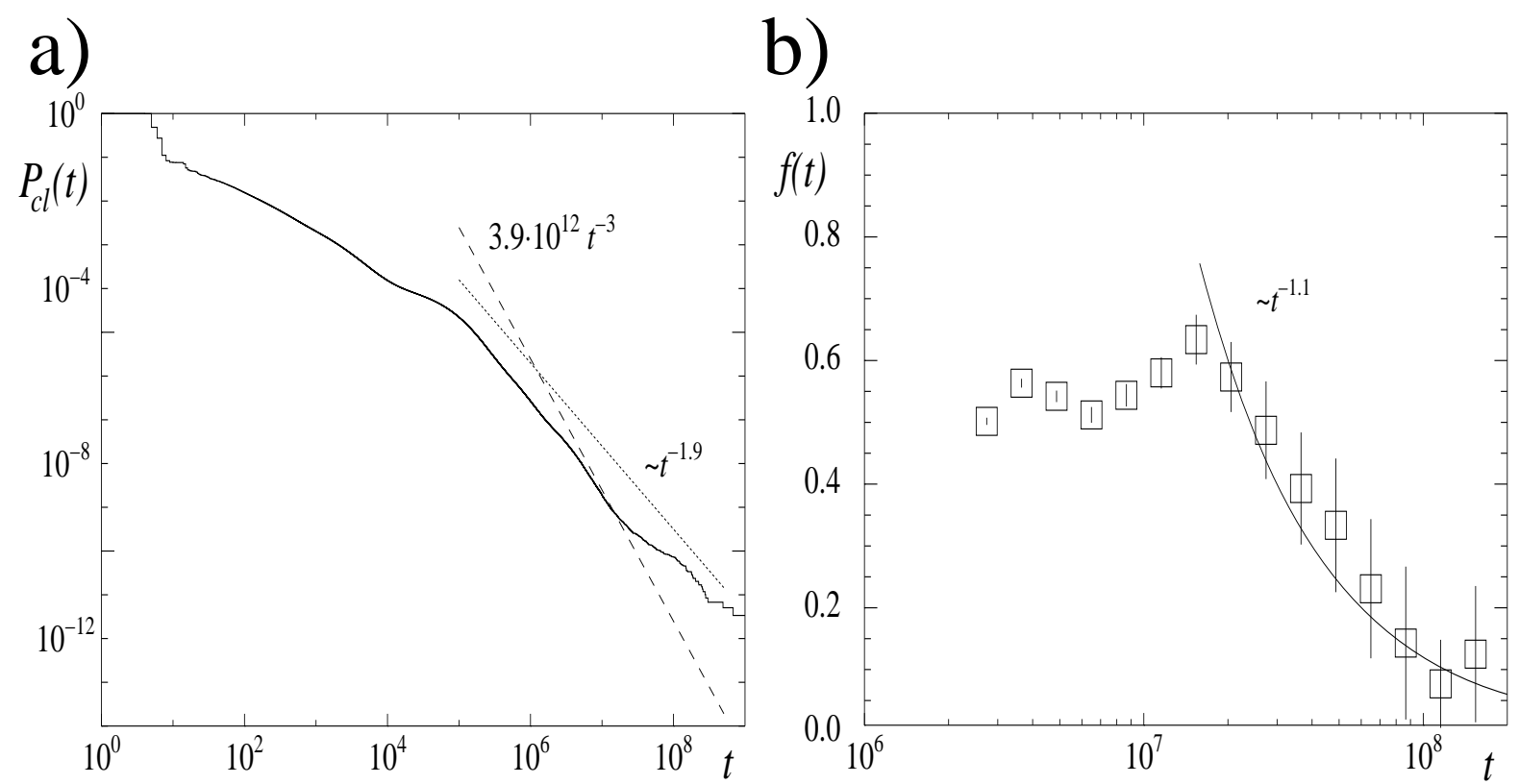

\begin{abstract}
Abbildung 3.2: a) Zerfall der klassischen Aufenthaltswahrscheinlichkeit für den gekickten Rotator bei $K=K_{c}$. Der Verlauf der numerisch bestimmten Kurve weicht deutlich von der Vorhersage aus Ref. [59] (gestrichelt) ab. b) Der relative Anteil $f(t)$ der Trajektorien, die in der selbstähnlichen Hierarchie zum goldenen Torus hängenbleiben, ist dargestellt. Für große Zeiten $\left(t>2 \cdot 10^{7}\right)$ tragen diese Trajektorien immer weniger zu $P(t)$ bei.
\end{abstract}

Zerfall [45,64], was zu zwei Erklärungsansätzen führte: Zum einem wurde spekuliert, dass das Hängenbleiben an nicht prinzipiellen Resonanzen wichtig sein könnte [50]. Dies würde zu einem schwächeren Zerfall mit $\gamma=1.96$ führen. Zum anderen wurde argumentiert, dass der asymptotische Zerfall erst zu sehr späten Zeiten einsetzt und die Numerik nur ein nicht universelles transientes Verhalten zeigt. Eine neuere Arbeit von Chirikov und Shepelyansky scheint die zweite Hypothese zu unterstützen [59]. Für Trajektorien mit Startbedingungen in der Nähe der hyperbolischen Fixpunkte der prinzipiellen Resonanzen $\left(p_{n} / q_{n}\right)$ haben sie die mittlere Zeit $\tau_{q_{n}}$ numerisch bestimmt, die die Trajektorien benötigen, um die Hierarchie zu verlassen. Die Analyse dieser mittleren Verweilzeiten liefert den asymptotischen Zerfall $P_{\mathrm{cl}}(t)=3.9 \cdot 10^{12} t^{-3}$ für Zeiten $t>10^{7}$, in Übereinstimmung mit den einfachen Renormierungstheorien, die die selbstähnliche Phasenraumstruktur ausnutzen.

Es ist jedoch äußerst fragwürdig, den Schluss von den mittleren Verweilzeiten auf den Zerfall der Aufenthaltswahrscheinlichkeit zu ziehen. Zwar kann die mittlere Verweilzeit Aufschluss darüber geben, zu welcher Zeit ein Bereich im Phasenraum zur Verteilung $P_{\mathrm{cl}}(t)$ beiträgt. Jedoch kann sie keine Auskunft darüber geben, wie wichtig der Beitrag des Bereichs im Vergleich zu anderen Bereichen ist. Um dies zu überprüfen, wurde die Verteilung $P_{\mathrm{cl}}(t)$ numerisch für den gekickten Rotator bei $K=K_{c}$ bestimmt. In einer kleinen Umgebung um den instabilen Fixpunkt $(q, p)=(0,0)$ wurden viele Trajektorien gestartet 


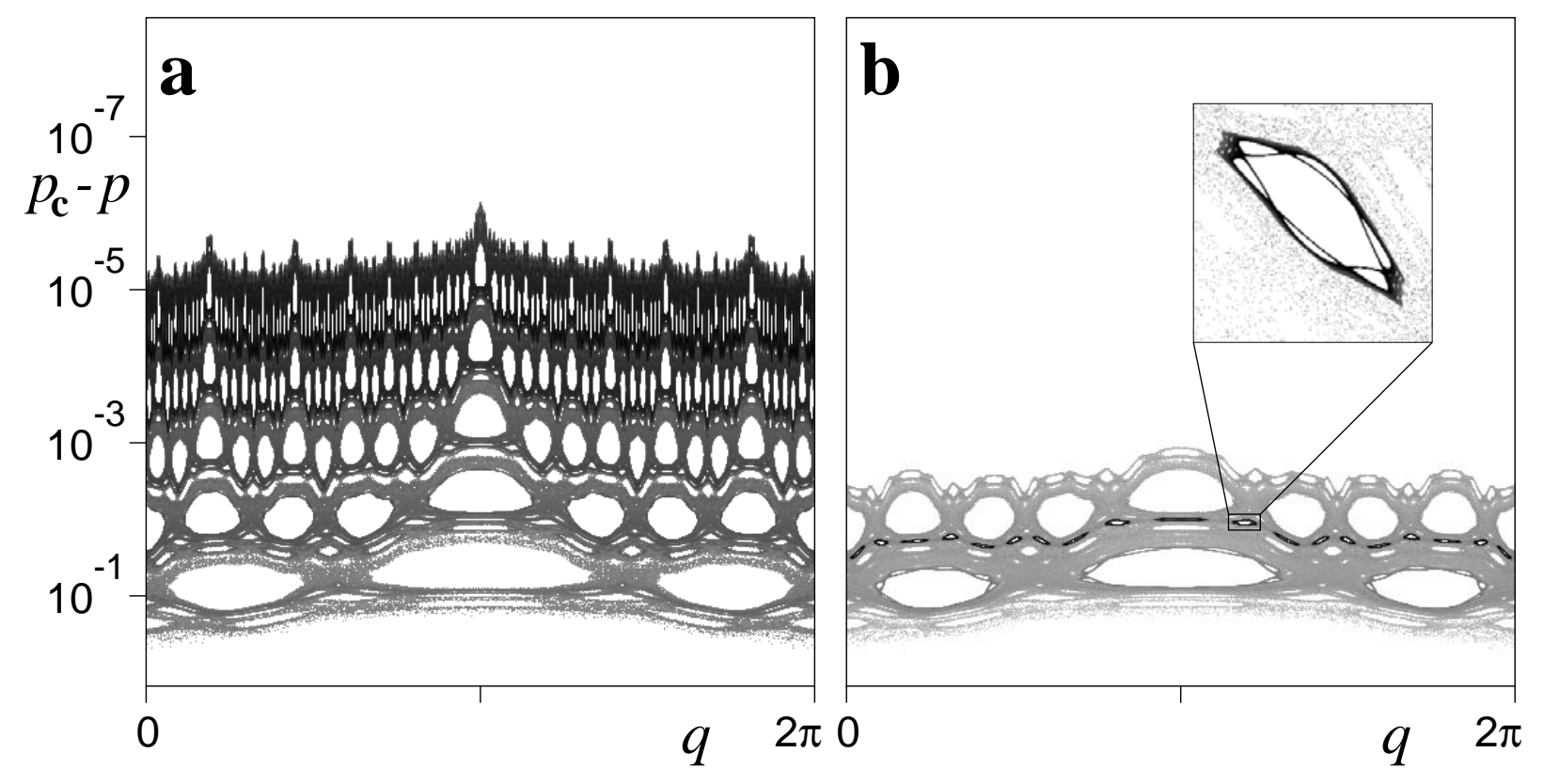

Abbildung 3.3: Phasenraumdichte für individuelle Trajektorien der Länge $t \approx 5 \cdot 10^{7}$ für $K=K_{c}$. Dabei ist für eine Trajektorie $(q, p)$ der Abstand $p_{c}(q)-p$ zum kritischen goldenen Torus $p_{c}(q)$ logarithmisch aufgetragen. a) Zeigt eine Trajektorie, die der selbstähnlichen Phasenraumstruktur folgt. b) Eine Trajektorie, die nicht in der Nähe des goldenen Torus hängenbleibt, sondern sich in nicht-prinzipalen Inselstrukturen aufhält. Die Phasenraumdichte wurde auf einem Gitter mit $500 \times 500$ Punkten bestimmt. Die Graustufen zeigen die Dichte auf einer logarithmischen Skala.

und für mehr als $10^{11}$ Schritte iteriert. Dabei kreuzt die Trajektorie mehrmals die Linie $p=0$. Die Anzahl der Iterationen zwischen zwei aufeinander folgenden Übertritten liefert die Aufenthaltszeit $\tau$ in der Hierarchie. Die lange Trajektorie setzt sich also aus vielen kürzeren Trajektorien zusammen, wobei der Endpunkt jedes Teils immer Startpunkt für das nachfolgende Segment ist. Die gesamte Computerzeit entsprach dabei $15 \cdot 10^{12}$ Iterationen des gekickten Rotators. In Abb. 3.2a ist die so ermittelte Verteilung zusammen mit der Vorhersage aus Ref. [59] dargestellt. Der Zerfall wird gut durch $\gamma \approx 1.9$ für große Zeiten beschrieben und weicht damit deutlich von der Vorhersage $\gamma=3 \mathrm{ab}$.

Um Aufschluss darüber zu erhalten, woher diese Abweichungen kommen, wurde für die Trajektorien mit Zeiten $\tau>3 \cdot 10^{6}$ untersucht, wo sie hängenbleiben. Dazu wurde für diese Trajektorien die Dichte im Phasenraum bestimmt. Damit die Strukturen in der Nähe des goldenen Torus besser aufgelöst werden, ist es sinnvoll, den Phasenraum zu entfalten. Dazu wird zunächst für jeden Punkt $(p(t), q(t))$ einer Trajektorie der Abstand $p_{c}(q(t))-p(t)$ zum goldenen Torus, der durch $p_{c}(q)$ beschrieben wird, berechnet. Die Dichte wird nun auf einem Gitter mit 500×500 Punkten bestimmt, wobei der Abstand zum Torus logarithmisch aufgetragen wird. Dadurch läßt sich gut feststellen, wo die Trajektorien im Phasenraum hängenbleiben. In Abb. 3.3a ist die Dichte für eine Trajektorie der Länge $5 \cdot 10^{7}$ gezeigt, 
die sich dem kritischen goldenen Torus nähert. In Übereinstimmung mit den in Ref. [59] ermittelten Verweilzeiten $\tau_{144}=4 \cdot 10^{7}$ dringt die Trajektorie bis zur prinzipiellen Resonanz $55 / 144$ vor. Abb. 3.3b zeigt eine Trajektorie, die ebenfalls $5 \cdot 10^{7}$ Zeitschritte in der Hierarchie verweilt, jedoch nicht über die prinzipielle Resonanz 5/8 hinauskommt und an einer nicht prinzipiellen Inselkette hängenbleibt. Diese Analyse wurde für alle Trajektorien mit Zeiten $\tau>3 \cdot 10^{6}$ durchgeführt. Dabei wird eine Trajektorie der Länge $\tau$ als konsistent mit der Renormierungstheorie angesehen, wenn sie bis zu der prinzipiellen Resonanz $p_{n} / q_{n}$ mit Verweilzeit $\tau_{q_{n}} \approx \tau$ vordringt. Nach dieser Methode wurden alle Trajektorien klassifiziert und der relative Anteil $f(t)$ der Trajektorien bestimmt, die in einem Zeitfenster um $t$ in Übereinstimmung mit der Renormierungstheorie sind. Wie man an dem Abfall von $f(t)$ für Zeiten $t>2 \cdot 10^{7}$ sieht, werden diese Trajektorien immer unwichtiger für das Verhalten von $P_{\mathrm{cl}}(t)$ (Abb. 3.2b). Dies zeigt deutlich, dass die Annahme der einfachen Renormierungstheorien, die selbstähnliche Struktur in der Nähe des kritischen Torus dominiere das Hängenbleiben, nicht erfüllt ist. Aus dem Verhältnis des vorhergesagten $P_{\mathrm{cl}}(t) \sim t^{-3}$ zum numerisch gefundenen $P_{\mathrm{cl}}(t) \sim t^{-1.9}$ erwartet man, dass der relative Anteil wie $f(t) \sim t^{-1.1}$ für große Zeiten abnimmt. Dies wird gut von den Daten in Abb. 3.2b unterstützt.

\subsection{Insel-um-Insel}

Im letzten Abschnitt wurde gezeigt, dass das Hängenbleiben in der Nähe eines kritischen noblen Torus nicht nur durch einen einfachen Renormierungspfad beschrieben werden kann. Diese Aussage soll nun anhand eines weiteren Beispiels untermauert werden. Dazu wird wieder der gekickte Rotator untersucht und zwar bei der Kickstärke $K=K^{*}=6.908745$. Der Phasenraum bei diesem Parameter ist fast vollständig chaotisch bis auf zwei kleine Bereiche regulärer Dynamik (Abb.3.4). Jeder Bereich besteht aus einer Hauptinsel mit drei Nebeninseln. Diese sind von acht Inseln umgeben, welche wiederum von einer Inselkette mit acht Inseln umgeben sind. Die Kickstärke wurde gerade so gewählt, dass die größten Inseln im Phasenraum, abgesehen von der Hauptinsel, immer von acht Tochterinseln umgeben sind [61]. Unter Verwendung renormierungstheoretischer Methoden, die das exakten Skalierungsverhalten ausnutzten, haben Zaslavsky et. al. für das Hängenbleiben chaotischer Trajektorien an den Inseln den klassischen Exponenten $\gamma=2.25$ vorhergesagt. Jedoch stellt sich auch hier die Frage, ob nicht - zumindest für große Zeiten - Bereiche wichtige Beiträge zum klassischen Zerfall liefern, die in der Renormierungstheorie nicht berücksichtigt werden.

Zur Klärung dieser Frage wurde der gekickte Rotator numerisch untersucht. Zunächst soll auf eine Besonderheit der Dynamik bei dieser Kickstärke eingegangen werden, die es erlaubt, einfach festzustellen, wie lange eine Trajektorie in der Nähe der regulären Bereiche hängenbleibt. Betrachtet man die Dynamik auf dem Zylinder $(p, q) \in \mathbb{R} \times S$, so findet man, dass die Inseln einer Zelle jeweils um $\pm 2 \pi$ in $p$-Richtung auf die Inseln der benachbarten Zellen abgebildet werden. Ein Teilchen, das auf einer Insel startet, vollzieht die Bewegung 


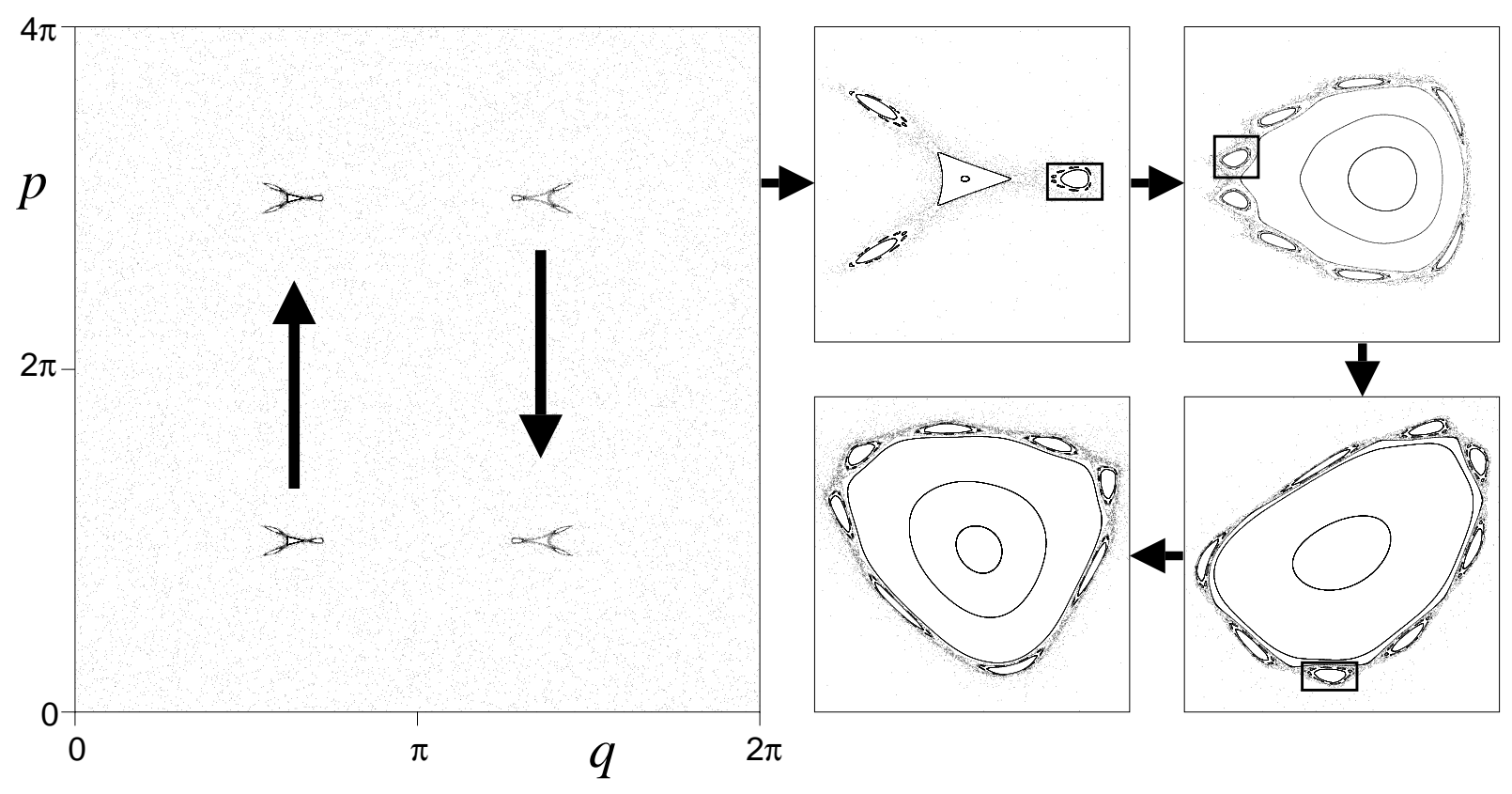

Abbildung 3.4: Phasenraum für den gekickten Rotator bei $K=K^{*}$ in symmetrischer Darstellung (nach einem halben Kick). Der Phasenraum ist fast vollständig chaotisch bis auf zwei kleine Beschleunigermoden. Die Pfeile geben jeweils die Richtung an, in die die Beschleunigermoden transportieren. Die sukzessiven Vergrößerungen zeigen die selbstähnliche Phasenraumstruktur: Die Hauptinsel wird von drei Nebeninseln umgeben. Diese werden auf der nächsten Stufe von acht Inseln umgeben, welche wiederum von einer Inselkette mit acht Inseln umrandet werden, usw. Insgesamt hat man die Sequenz $3-8-8 \ldots$

auf einem Torus der Insel, wobei es bei jeder Iteration eine Zelle weiter hüpft. Auf Grund dieser Eigenschaft bezeichnet man die beiden Ketten regulärer Dynamik auch als Beschleunigermoden. Eine chaotische Trajektorie hüpft solange mit der Beschleunigermode, wie sie an ihren Inselstrukturen hängenbleibt. Eine Trajektorie mit Startpunkt in der chaotischen See wird nun solange evolviert, wie sie kontinuierlich bei jedem Zeitschritt eine Einheitszelle weiter springt. Die Anzahl der Iterationen liefert dann die Verweilzeit $\tau$ der Trajektorie in der Hierarchie der regulären Bereiche.

In Abb. 3.5 ist die numerisch ermittelte Verteilung der Aufenthaltszeiten $P_{\mathrm{cl}}(t)$ für die beiden Beschleunigermoden gezeigt. Zunächst wurden $\approx 5 \cdot 10^{11}$ Teilchen auf einer Linie $p=$ const außerhalb der Beschleunigermoden gestartet. Die längste Trajektorie verweilt $5 \cdot 10^{6}$ Iterationen in der Nähe der Beschleunigermoden. Dies ist zwar schon um fast eine Größenordnung länger als die Untersuchungen in Ref. [67]. Will man jedoch noch Aussagen für spätere Zeiten bekommen, so ist es nicht zweckmäßig, außerhalb der Beschleunigermoden zu starten. Dies liegt darin begründet, dass die Beschleunigermoden nur einen sehr geringen Teil des Phasenraums einnehmen und daher viele Teilchen erst gar nicht in die Nähe der Moden gelangen. Um also auch Aussagen für große Zeiten zu erhalten, wurden 

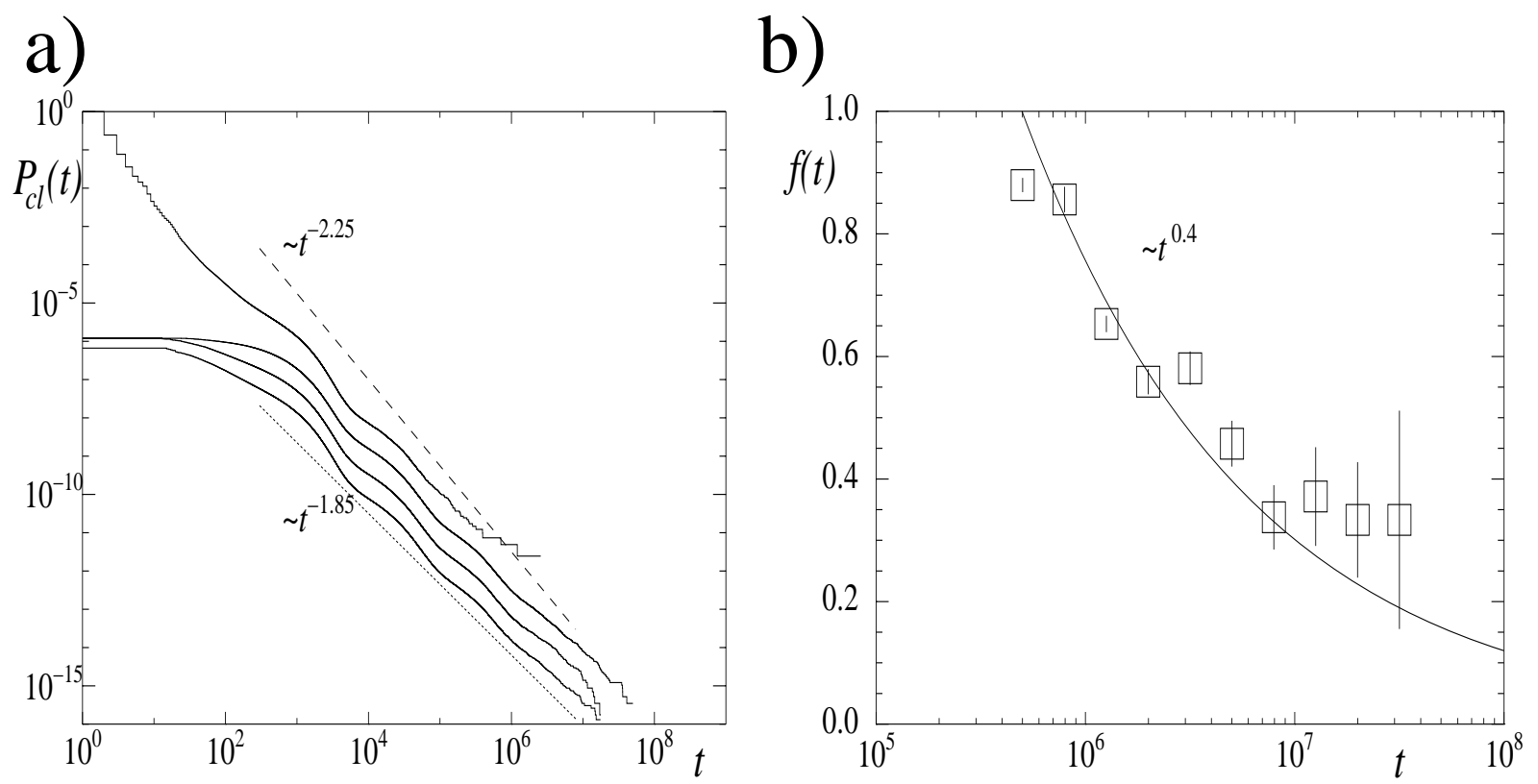

\begin{abstract}
Abbildung 3.5: a) Gezeigt ist der Zerfall $P_{\mathrm{cl}}(t)$ für $K=K^{*}$ verschiedene Anfangsbedingungen (s. Text). Alle vier Kurven zeigen das selbe Potenzgesetz (inklusive der Fluktuationen), was von der Vorhersage $\gamma=2.25$ aus Ref. [61] (gestrichelt) abweicht. b) Für die längsten Trajektorien $\left(\tau>5 \cdot 10^{6}\right)$ ist der relative Anteil $f(t)$ der Trajektorien gezeigt, die der selbstähnlichen Inselum-Insel Struktur folgen. Der Abfall wird gut durch das Verhältnis des vorhergesagten zum gefundenen Zerfall beschrieben (durchgezogene Linie).
\end{abstract}

Trajektorien an drei verschiedenen Stellen in der Nähe einer Beschleunigermode gestartet. Natürlich müssen die so bestimmten Verteilungen für kleine Zeiten von der Verteilung mit Anfangsbedingungen $p=$ const abweichen, da jede Trajektorie zumindest einige Iterationen in der Nähe der Beschleunigermode verweilt. Für Zeiten $t>10^{3}$ zeigen jedoch alle vier Verteilungen den gleichen Zerfall. Nicht nur der Exponent stimmt überein, sondern auch die Fluktuationen um diesen mittleren Zerfall werden von allen Verteilungen gleichermaßen reproduziert. Das zeigt deutlich, dass die gewählten Startbedingungen verlässliche Ergebnisse für $P_{\mathrm{cl}}(t)$ liefern. So erreicht man leicht Zeiten von $t \approx 5 \cdot 10^{7}$ und damit zwei Größenordnungen weiter als in Ref. [67]. Der Zerfall wird gut durch den Exponenten $\gamma=1.85$ für Zeiten $t>10^{3}$ beschrieben und weicht damit, zwar nicht so extrem wie beim kritischen goldenen Torus, aber immer noch deutlich von der theoretischen Vorhersage $\gamma=2.25 \mathrm{ab}$. Auch für die Beschleunigermoden wurde untersucht, wo die längsten Trajektorien hängenbleiben. In Abb. 3.6 sind die Phasenraumdichten zweier Trajektorien, die $t \approx 5 \cdot 10^{7}$ Iterationen einer Beschleunigermode folgen, gezeigt. Abb. 3.6a zeigt die Dichte einer Trajektorie, die dem selbstähnlichen Insel-um-Insel Pfad folgt. Im Gegensatz dazu ist in Abb.3.6b die Dichte einer Trajektorie wiedergegeben, die an anderen Inselstrukturen im Phasenraum hängenbleibt. Dass diese weiteren Inselstrukturen für große Zeiten wichtig werden, zeigt Abb.3.5b, in der der relative Anteil $f(t)$ der Trajektorien darge- 

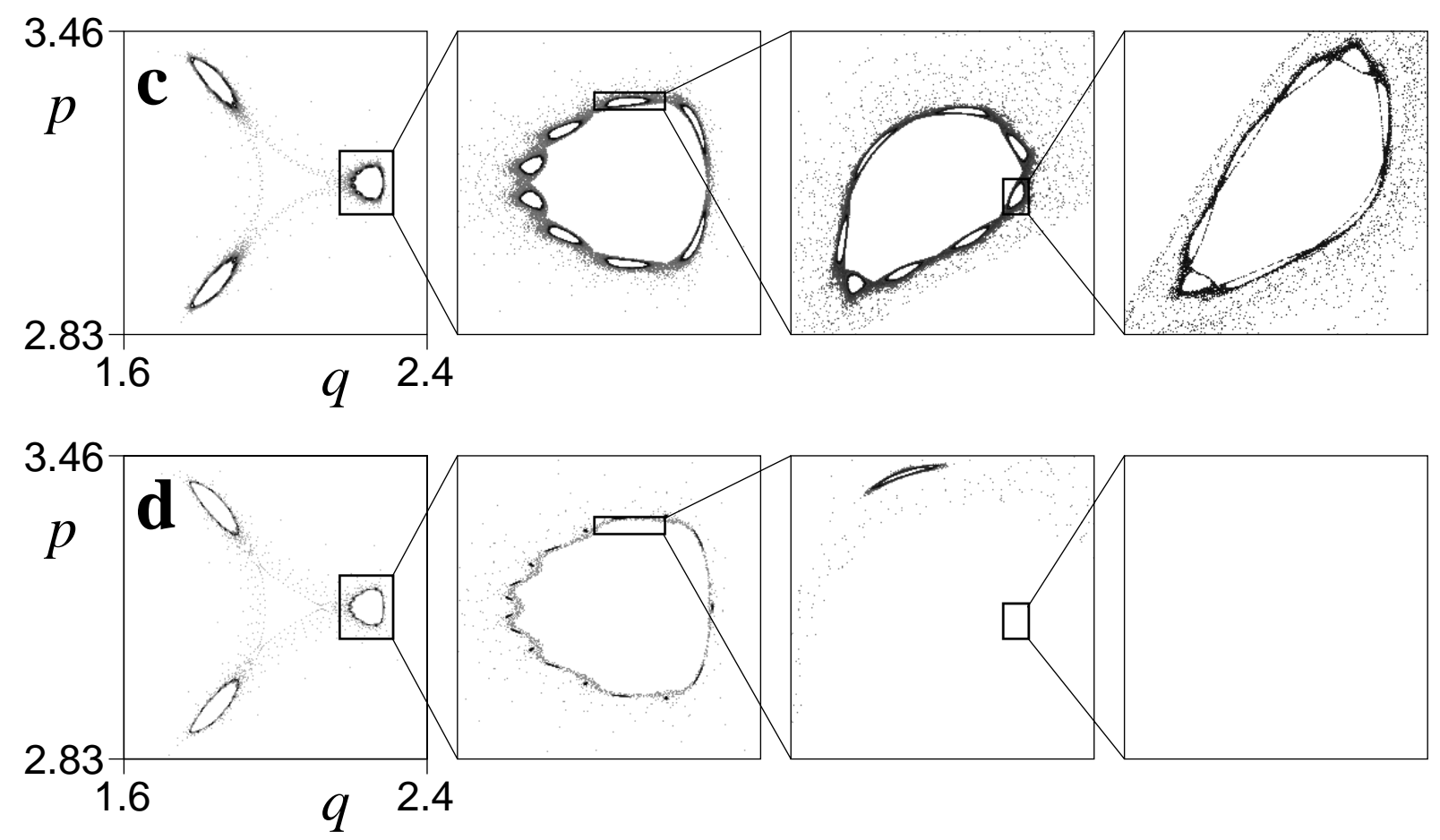

Abbildung 3.6: Dichte im Phasenraum für zwei Trajektorien der Länge $\approx 5 \cdot 10^{7}$ mit jeweiligen Vergrößerungen um die selbstähnliche Inselkette. a) Gezeigt ist eine Trajektorie, die der selbstähnlichen Struktur folgt. b) Zeigt eine Trajektorie, die an Inselstrukturen, die nicht von der Renormierungstheorie berücksichtigt werden, hängenbleibt.

stellt ist, die dem selbstähnlichen Insel-um-Insel Pfad folgen. Für Zeiten $t>10^{6}$ bleibt ein immer geringerer Anteil in der ..8 $8-8-\ldots$ Sequenz hängen. Wie schon beim kritischen goldenen Torus wird auch hier wieder der Abfall von $f(t)$ näherungsweise durch das Potenzgesetz $f(t) \sim t^{-0.4}$ beschrieben, das man aus dem Verhältnis des theoretisch vorhergesagten Zerfalls $P_{\mathrm{cl}}(t) \sim t^{-2.25}$ zum numerisch bestimmten $P_{\mathrm{cl}}(t) \sim t^{-1.85}$ findet. Dies zeigt, dass die einfache Renormierungstheorie zwar das Hängenbleiben in der selbstähnlichen Insel-um-Insel Struktur beschreibt, diese aber nur einen Teilbeitrag zu $P_{\mathrm{cl}}(t)$ liefert, der darüberhinaus für große Zeiten immer unwichtiger wird.

Es soll nun noch kurz auf eine Konsequenz, die sich aus den o.a. Resultaten zu den Beschleunigermoden ergibt, hingewiesen werden. Es ist eine lang bekannte Tatsache, dass die spezielle Dynamik der Beschleunigermoden zu anomaler Diffusion führt, wenn das Hängenbleiben an den Beschleunigermoden mit Exponenten $\gamma<2$ zerfällt. [68-70]. Anomale Diffusionsprozesse zeichnen sich dadurch aus, dass die Varianz eines Ensembles von Trajektorien nicht linear mit der Zeit, sondern mit einem Exponenten $1<\mu<2$ anwächst. Zaslavsky et. al. behaupten, dass sie für $K=K^{*}$ anomale Diffusion finden, was auch durch ihre numerischen Untersuchungen unterstützt wird [61]. Das passt jedoch nicht zu dem vorhergesagten Exponenten $\gamma=2.25$, wonach man "normale" Diffusion erwartete. Dieser Widerspruch hat 
zu einiger Verwirrung in der Literatur geführt [71,72], der mit den obigen Ergebnissen nun ausgeräumt ist.

\subsection{Zusammenfassung und Ausblick}

In diesem Kapitel wurde das Hängenbleiben chaotischer Trajektorien in der Nähe der regulären Inseln numerisch für den gekickten Rotator zu zwei Kickstärken $K_{c}$ und $K^{*}$ eingehend untersucht. Beide Kickstärken zeichnen sich dadurch aus, dass es im Phasenraum jeweils einen Pfad gibt, entlang dem der Phasenraum eine exakt selbstähnliche Struktur aufweist: Für $K_{c}$ ist dies der Pfad zum kritischen goldenen Torus und für $K^{*}$ gibt es eine Insel-um-Insel Sequenz, die selbstähnlich ist. Diese Selbstähnlichkeit wurde verwendet, um mit Hilfe von renormierungstheoretischen Methoden den klassischen Exponenten $\gamma$ für das Hängenbleiben an den Inseln zu berechnen $[53,61,63,66]$. Jedoch findet man numerisch für beide Parameter erhebliche Abweichungen von diesen Vorhersagen. Dies liegt nicht daran, dass die Theorien die selbstähnlichen Strukturen falsch beschreiben, sondern, wie hier gezeigt wurde, dass Trajektorien an anderen Strukturen im Phasenraum hängenbleiben. Die genaue Analyse der Phasenraumdichten der längsten Trajektorien zeigt darüber hinaus, dass diese anderen Bereiche für große Zeiten immer wichtiger werden. Die Ergebnisse dieses Kapitels widerlegen die Behauptung, der universelle Exponent für den asymptotische Zerfall sei $\gamma=3[59]$.

Obwohl diese eindimensionalen Renormierungstheorien ein qualitatives Verständnis der Dynamik in gemischten Systemen liefern, muss eine quantitative Beschreibung die komplette Phasenraumstruktur berücksichtigen. Erste Ansätze dazu gehen auf Meiss und Ott zurück, die ein Baummodell für die Dynamik vorgeschlagen haben [50]. Die Suche nach dem universellen Exponenten, so er denn existiert, kommt um eine detailiertere Untersuchung der Eigenschaften dieses Modells nicht herum. Im Allgemeinen weist der Phasenraum keine exakte Skalierung auf, sondern ist nur im statistischen Sinn selbstähnlich. Ein vielversprechendes Modell für realistische Phasenraumstrukturen könnte daher ein Baum mit Unordnung in den Übergangsraten sein. 


\section{Kapitel 4}

\section{Hierarchische Eigenzustände}

In diesem Kapitel soll der Einfluss der klassischen Transportbarrieren auf Spektrum und Eigenfunktionen eines Quantensystems mit gemischtem Phasenraum herausgearbeitet werden. Das Hauptergebnis dieser Untersuchungen ist die Charakterisierung und Quantifizierung einer neuen Klasse von Eigenzuständen [38].

\subsection{Eine neue Klasse von Eigenzuständen}

Der klassische Phasenraum eines gemischten Systems unterteilt sich in Regionen regulärer und chaotischer Dynamik. Für Eigenfunktion und Spektrum des korrespondierenden HamiltonOperators hat Percival [32] eine entsprechende Zweiteilung in regulär und chaotisch vorgeschlagen. Die regulären Zustände zeichnen die invarianten KAM-Tori der Inseln nach. Wie bei integrablen Systemen sind reguläre Eigenenergien und Eigenfunktionen im Rahmen der Einstein-Brillouin-Keller (EBK) [18-20] Quantisierung verständlich. Ist $C$ ein KAM-Torus im Poincaré-Schnitt und erfüllt die Wirkung die Quantisierungsbedingung

$$
J=\oint_{C} p \mathrm{~d} q=2 \pi \hbar\left(n+\frac{1}{2}\right)
$$

mit einer ganzen Zahl $n \geq 0$, dann besitzt - zumindest im semiklassischen Limes - der Hamilton-Operator einen Eigenwert, dessen Wert der klassischen Energie auf dem Torus $C$ entspricht. Motiviert durch die Untersuchungen vollständig chaotischer Systeme werden chaotische Zustände im gemischten Phasenraum dadurch charakterisiert, dass sie als Träger die chaotische Komponente des Phasenraums haben und abgesehen von Fluktuationen den Träger gleichmäßig bedecken. Die Fluktuationen um die mittlere Anregungsstärke folgen in guter Näherung der Theorie der Zufallsmatrizen.

Die Annahme, dass sich die chaotischen Zustände gleichmäßig auf der chaotischen Kom- 


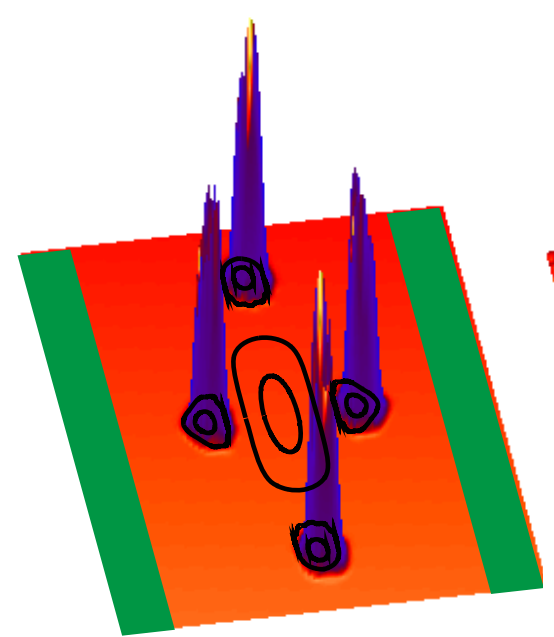

regular

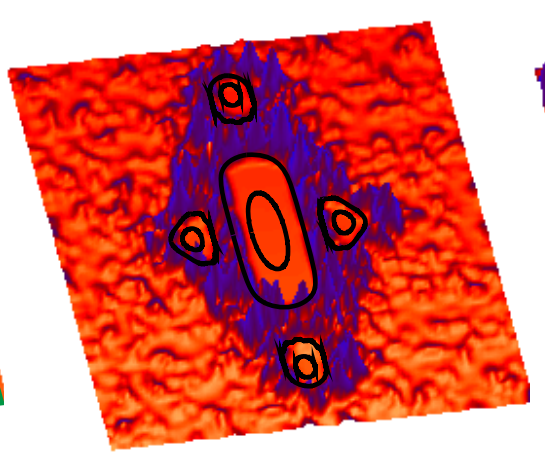

hierarchical

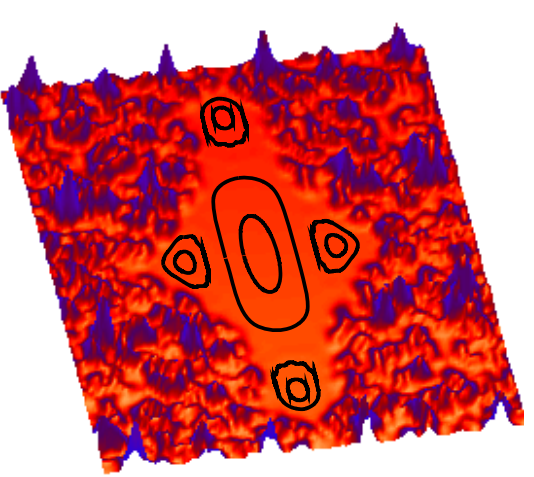

chaotic

\begin{abstract}
Abbildung 4.1: Beispiele für einen regulären, hierarchischen und chaotischen Zustand der Standardabbildung ( $K=2.5, \hbar=2 \pi / 1000)$. Die eingetragenen Linien skizzieren die Lage der invarianten Tori des zugehörigen Phasenraums.
\end{abstract}

ponente der klassischen Dynamik verteilen, berücksichtigt nicht die Existenz der partiellen Transportbarrieren, die einen starken Einfluss auf die Dynamik haben. Im Folgenden wird gezeigt, dass die partiellen Transportbarrieren zu einer neuen Klasse von Eigenfunktionen führen, den hierarchischen Eigenfunktionen. Diese Zustände haben als Träger die chaotisch invariante Menge, leben jedoch in der Nähe der Inseln und haben nur kleine Beiträge in der chaotischen See. In Abb. 4.1 ist jeweils eine typische reguläre, hierarchische und chaotische Eigenfunktion dargestellt.

In der klassischen Hierarchie des gemischten Phasenraums werden die Flüsse durch die partiellen Transportbarrieren beliebig klein, was zu dem charakteristischen algebraischen Zerfall führt. Der Fluss durch eine partielle Transportbarriere ist gegeben durch die Fläche des turnstile. Quantenmechanisch können jedoch nur Strukturen im Phasenraum aufgelöst werden, die größer als $\hbar$ sind. Turnstiles, deren Flächen kleiner als $\hbar$ ist, werden demnach nicht aufgelöst und quantenmechanisch wirkt die partielle Transportbarriere wie ein geschlossener Torus, der zwei Regionen separiert. Die partielle Transportbarriere, deren Fluss $\Phi$ gleich $\hbar$ ist, nennt man Flussbarriere. Die Flussbarriere unterteilt die chaotische Komponente der Dynamik in zwei Bereiche: Die Regionen, die durch partielle Transportbarrieren verbunden sind, deren Fluss größer als $\hbar$ ist, sind stark gekoppelt und wirken quantenmechanisch als eine zusammenhängende Region. Hingegen sind die Regionen, die durch Flüsse kleiner als $\hbar$ verbunden sind, schwach gekoppelt. Solange das Volumen einer Region, die schwach an die benachbarten Regionen koppelt, groß gegenüber $\hbar$ ist, existie- 
ren Eigenzustände, die in der Region konzentriert sind. Dies sind gerade die hierarchischen Eigenfunktionen. Für $\hbar \rightarrow 0$ rückt die Flussbarriere immer näher an die Inseln und die hierarchischen Zustände leben immer tiefer in der klassischen Hierarchie. Deshalb werden diese Wellenfunktionen als hierarchisch bezeichnet.

\subsection{Der Anteil der hierarchischen Eigenzustände}

Es soll nun untersucht werden, wie sich der Anteil der hierarchischen Wellenfunktionen mit $\hbar$ verändert. Seien $\rho_{\text {reg }}$ und $\rho_{\text {ch }}$ die relativen Volumina der regulären Inseln und der chaotischen Komponente. Für ein bestimmtes $\hbar$ sei der relative Anteil regulärer, hierarchischer und chaotischer Eigenfunktionen durch $f_{\text {hier }}(\hbar), f_{\text {reg }}(\hbar)$ und $f_{\text {ch }}(\hbar)$ bezeichnet. Dann muss offensichtlich gelten

$$
f_{\text {reg }}(\hbar)+f_{\text {hier }}(\hbar)+f_{\text {ch }}(\hbar)=1=\rho_{\text {reg }}+\rho_{\text {ch }} \quad .
$$

Für hinreichend kleines $\hbar$ wird die Anzahl der regulären Zustände allein durch die Größe der regulären Bereiche bestimmt. In guter Näherung ist dann $f_{\text {reg }}=\rho_{\text {reg }}$, was mit Hilfe von Gl. (4.2) zu

$$
f_{\text {hier }}=\rho_{\text {ch }}-f_{\text {ch }}
$$

führt, d.h. gemeinsam haben die chaotischen und hierarchischen Zustände die chaotische invariante Menge als Träger. Ist $\rho_{\mathrm{ch}}$ bekannt, so liefert Gl. (4.3) eine Beziehung zwischen dem Anteil der hierarchischen und chaotischen Eigenfunktionen. Die Größe der chaotischen Komponenten kann auf zwei Weisen bestimmt werden. Zur exakten Bestimmung von $\rho_{\text {ch }}$ müsste man die Größe aller Inseln im Phasenraum bestimmen, was natürlich unmöglich ist. Dennoch lässt sich $\rho_{\text {ch }}$ gut näherungsweise bestimmen. Zwei Verfahren sind dabei naheliegend: Zum einen kann man die Fläche aller Inseln bestimmen, die größer als eine Box mit Flächen $\varepsilon$ sind. Zum anderen kann man eine große Anzahl von Trajektorien mit Startpunkt in der chaotischen See über viele Zeitschritte iterieren, um dann die Fläche zu bestimmen, die diese Trajektorien bedecken. Dazu unterteilt man den Phasenraum in Boxen mit der Fläche $\varepsilon$ und bestimmt die relative Anzahl der Boxen, die von einer Trajektorie besucht werden. Beide Vorgehensweisen haben gemeinsam, dass sie für $\varepsilon \rightarrow 0$ das exakte Ergebnis liefern und der Fehler für ein kleines $\varepsilon>0$ gut abgeschätzt werden kann. Ist nun $\rho_{\text {reg }}$ bekannt, so kann mit Hilfe von Gl. (4.3) der relative Anteil der hierarchischen Eigenfunktionen bestimmt werden.

\subsubsection{Einfluss auf die Eigenfunktionen}

Ohne hierarchische Eigenfunktionen stünde für die chaotischen Zustände die gesamte chaotische Komponente $\rho_{\text {ch }}$ zur Verfügung. Unter der Annahme, dass sich die chaotischen 
Zustände hierauf gleichmäßig verteilen, beträge dann die mittlere Anregungsstärke $1 / \rho_{\mathrm{ch}}$. Die Existenz der hierarchischen Eigenfunktionen reduziert die Region, auf der die chaotischen Zustände leben, was zur Folge hat, dass die mittlere Anregungsstärke in der chaotischen See ansteigt. Sei $\Omega^{\prime}$ ein Volumen in der chaotischen See, welches groß gegenüber $\hbar$ ist. Die mittlere Anregungsstärke $\eta$ in diesem Volumen wird definiert durch

$$
\eta=\frac{\Omega \cdot \rho_{\mathrm{ch}}}{\Omega^{\prime}} \int_{\Omega^{\prime}} \mathrm{d} p \mathrm{~d} q|\psi(p, q)|^{2}
$$

wobei $\Omega$ das Gesamtvolumen des Phasenraums ist und $\psi(p, q)$ die Husimi-Darstellung der Wellenfunktion ist. Die Skalierung ist so gewählt, dass eine Wellenfunktion, die gleichmäßig die chaotische Komponente des Phasenraums überdeckte, die mittlere Anregungsstärke $<\eta>=1$ hätte.

In Abb. 4.2 ist ein Beispiel für die Verteilung $P(\eta)$ gezeigt. Die Beiträge der Inseln sorgen für viele kleine $\eta$. Die chaotischen Eigenfunktionen sorgen für große $\eta$, die sich in guter Näherung gaußförmig um den Maximalwert $\rho_{\mathrm{ch}} / f_{\mathrm{ch}}$ verteilen. Aus $\rho_{\mathrm{ch}} / f_{\mathrm{ch}}>1$ folgt, dass die chaotischen Eigenfunktionen auf einem Volumen leben, was kleiner als $\Omega \rho_{\text {ch }}$ ist. Aus der Position des Maximalwertes kann nun unter Verwendung von Gl. (4.3) der Anteil der hierarchischen Eigenfunktionen berechnet werden. Eine Auswertung mehrerer solcher Verteilungen für verschiedene $\hbar$ liefert die gesuchte funktionale Abhängigkeit $f_{\text {hier }}(\hbar)$.

\subsubsection{Einfluss auf das Spektrum}

Auch der Einfluss der hierarchischen Eigenfunktionen auf das Spektrum kann genutzt werden, um ihren relativen Anteil zu bestimmen. Berry und Robnik haben vorgeschlagen, dass man das Spektrum gemischter Systeme als unabhängige Überlagerung von Eigenwerten regulärer und chaotischer Zustände erhält [33]. Die Statistik nächster Niveauabstände der chaotischen Wellenfunktionen folgt der Zufallsmatrizentheorie, ist also GOE-verteilt für Systeme mit Zeitumkehrsymmetrie. Die Abstände der regulären Zustände sind hingegen Poisson verteilt. Eine unabhängige Überlagerung beider Spektren wird beschrieben durch die Berry-Robnik-Verteilung $p_{\mathrm{BR}}\left(f_{\mathrm{BR}}, s\right)$, wobei $s$ der Niveauabstand ist und $f_{\mathrm{BR}}$ den Anteil der regulären Zustände angibt [33]. Dieser Ansatz berücksichtigt nicht die Existenz der hierarchischen Zustände. Da die Wechselwirkung zwischen den hierarchischen Zuständen sehr schwach ist, erhält man das Spektrum der hierarchischen Eigenfunktionen als unabhängige Überlagerung einzelner GOE-Spektren. Jedem Teilspektrum entspricht eine Region im Phasenraum, auf der hierarchische Zustände konzentriert sind. Der mittlere Niveauabstand der hierarchischen Zustände ist $1 / f_{\text {hier }}$ und ist typischerweise klein gegenüber dem mittleren Niveauabstand des gesamten Spektrums. Da weiterhin die hierarchischen Zustände nur schwach an die chaotischen und regulären Bereiche koppeln, liegen ihre Eigenwerte zufällig verteilt im Spektrum. Sie erhöhen damit den Poisson-Anteil, d.h. das Spektrum folgt weiterhin der Berry-Robnik Verteilung, allerdings muss der Parameter $f_{\mathrm{BR}}$ um die 

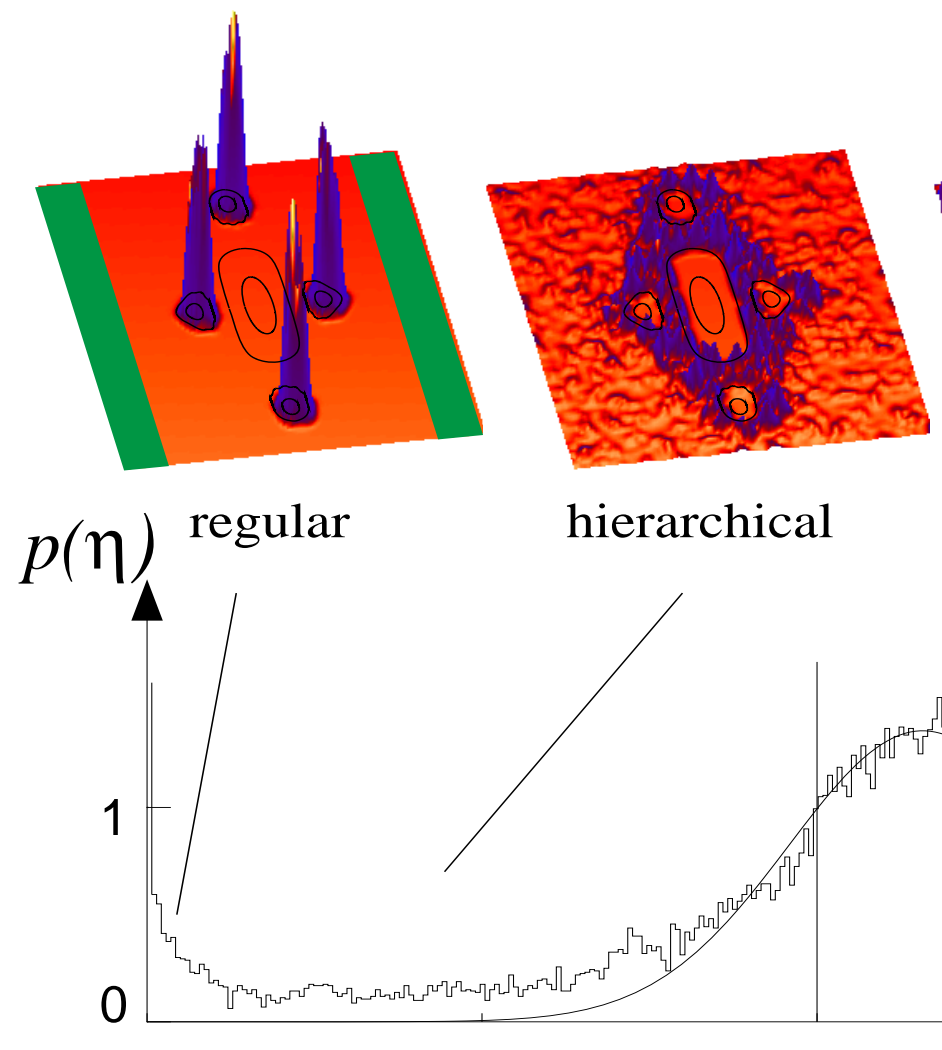

hierarchical

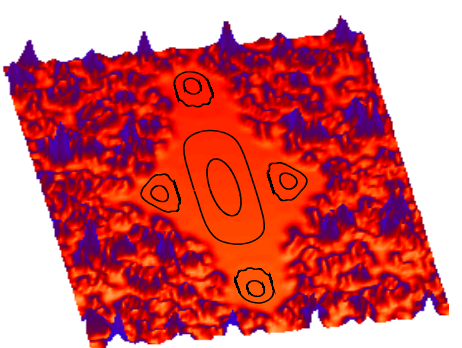

chaotic

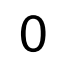

0.5
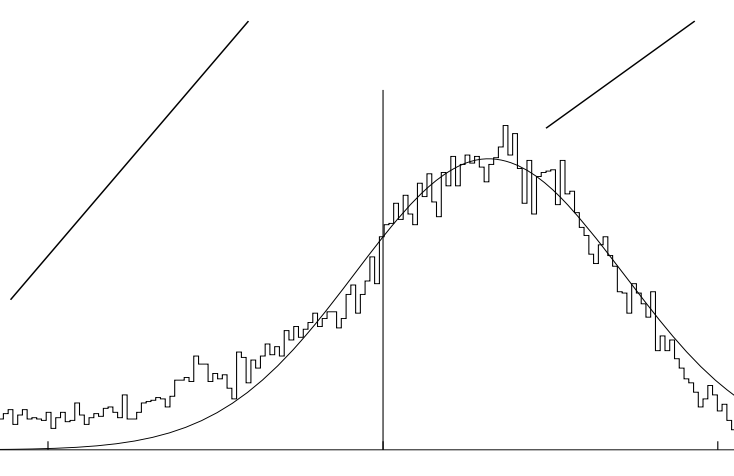

Abbildung 4.2: Statistik der Anregungsstärken $P(\eta)$ für die Standardabbildung $(K=2.5,2 \pi \hbar=$ 1/1000). Der Erwartungswert des eingezeichneten Gauß-Fit liegt deutlich oberhalb der 1.0. Oben aufgetragen sind nochmals die drei generischen Eigenfunktionen mit Verweis auf den Teil der Verteilung $P(\eta)$, zu dem sie beitragen. Der zur Bestimmung von $\eta$ verwendete Bereich ist grün unterlegt.

hierarchischen Zustände ergänzt werden, $f_{\mathrm{BR}}=f_{\text {reg }}+f_{\text {hier }}$. Den Parameter $f_{\mathrm{BR}}$ erhält man als Fit-Parameter der Abstandsverteilung und mit

$$
f_{\text {hier }}=f_{\mathrm{BR}}-\rho_{\text {reg }}
$$

kann der Anteil der hierarchischen Eigenfunktionen bestimmt werden.

Die Daten beider Methoden sind in Abb. 4.3 als Funktion von $\hbar$ und zu drei Kickstärken zusammen mit dem klassischen $P_{\mathrm{cl}}(t)$ dargestellt. Deutlich ist zu sehen, dass der relative Anteil der hierarchischen Eigenfunktionen für $\hbar \rightarrow 0$ algebraisch abnimmt. Die Abnahme wird in guter Näherung durch die Gleichung

$$
f_{\text {hier }} \sim \hbar^{1-1 / \gamma}
$$




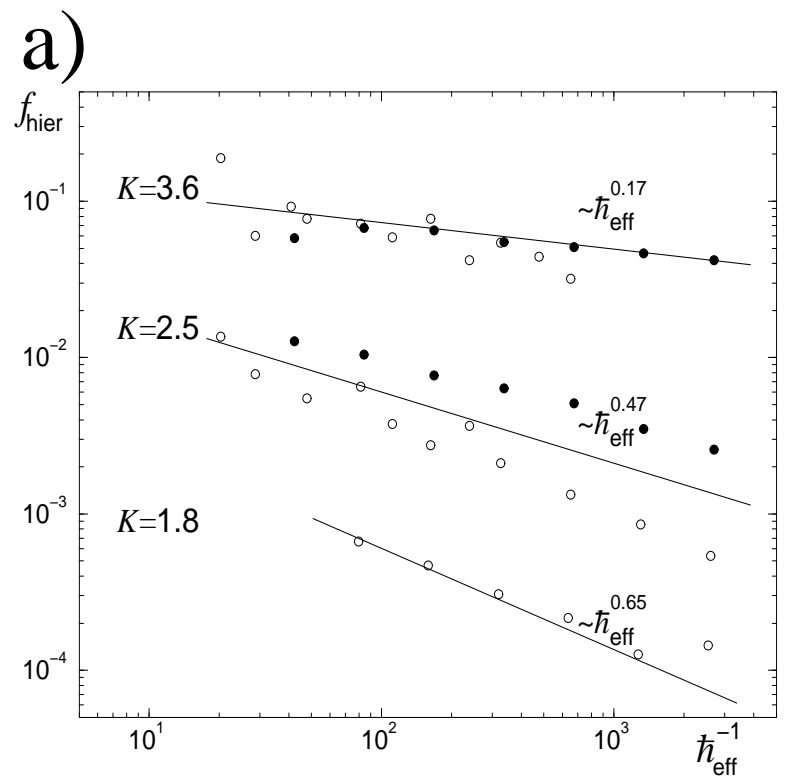

b)

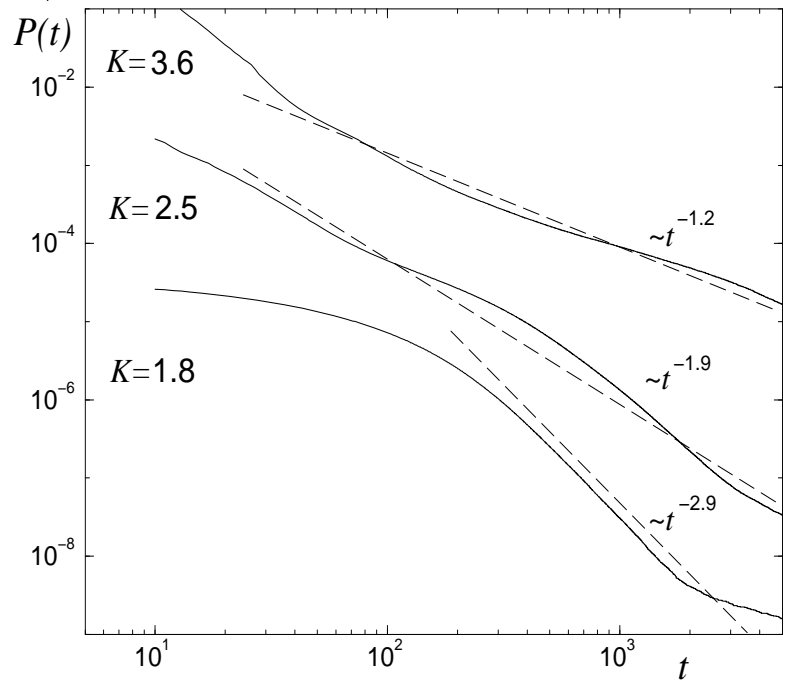

Abbildung 4.3: a) Skalierung von $f_{\text {hier }}$ mit $\hbar_{\text {eff }}$ für drei verschiedene Kickstärken $(K=$ 3.6, 2.5 und 1.8) der Standardabbildung. Die vollen Punkte wurden mit Hilfe der $\eta$-Statistik, die Rauten über einen Fit der Berry-Robnik-Statistik ermittelt. Die Vorhersage gemäß Gl. (4.11) ist durchgezogen eingezeichnet, wobei der klassische Exponent aus b) stammt. b) Klassisches $P_{\mathrm{cl}}(t)$ für die drei Kickstärken mit den gefitteten Potenzgesetzen.

beschrieben, wobei $\gamma$ der Exponent des Zerfalls der klassischen Aufenthaltswahrscheinlichkeit $\left(P_{\mathrm{cl}}(t) \sim t^{-\gamma}\right)$ ist. Dass die beiden Methoden leicht unterschiedliche Werte liefern, sollte nicht überraschen, da beide Verfahren auf Annahmen beruhen, die unterschiedlich gut erfüllt sind. Entscheidend ist jedoch, das beide Datensätze denselben Exponenten liefern. Warum der Anteil der hierarchischen Eigenfunktionen algebraisch abnimmt und woher die Abhängigkeit von der klassischen Dynamik stammt, soll nun an einem einfachen Modell gezeigt werden.

\subsection{Quantenmechanik des Kettenmodells}

Im Abschnitt 2.4.3 wurde gezeigt, dass sich die Dynamik im gemischten Phasenraum qualitativ durch das Kettenmodell beschreiben lässt. Es besteht aus einer Reihe von Volumina $\Omega_{n}$, die durch Flüsse $\Phi_{n, n+1}$ paarweise verbunden sind. In Abb. 4.4 ist dies schematisch dargestellt. Unter der Annahme, dass die Volumina und die Flüsse skalieren

$$
\Omega_{n}=\Omega_{0} \omega^{n} \quad \text { und } \quad \Phi_{n, n+1}=\Phi_{0} \phi^{n} \quad,
$$

lässt sich der Exponent des algebraischen Zerfalls $P_{\mathrm{cl}}(t) \sim t^{-\gamma}$ gemäß

$$
\gamma=1 /(1-\ln \omega / \ln \phi)
$$




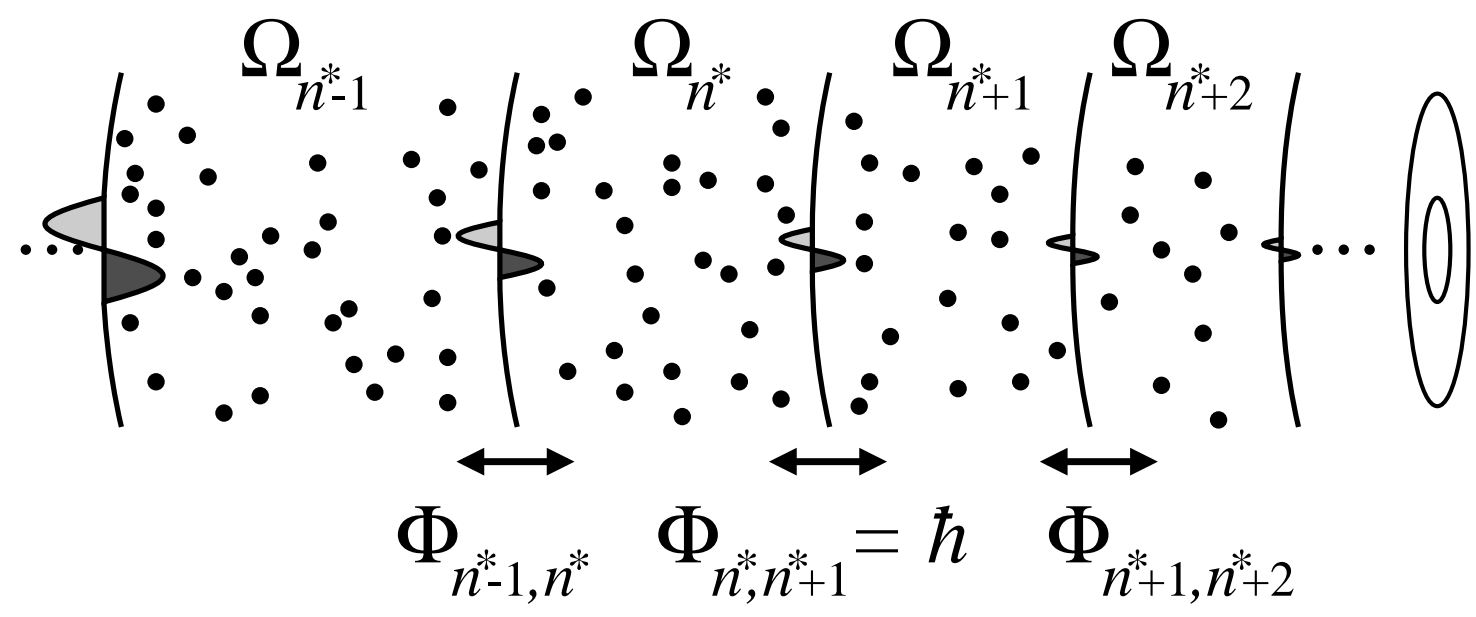

Abbildung 4.4: Skizze des gemischten Phasenraums um die Flussbarriere. Die Volumina $\Omega_{n}$ sind durch partielle Transportbarrieren getrennt und der Phasenraumaustausch (turnstile) zwischen zwei benachbarten Regionen ist durch den Fluss $\Phi_{n, n+1}$ gegeben. Die Flussbarriere trennt die Regionen $n^{*}$ und $n^{*}+1$.

berechnen. Die Lage der Flussbarriere $n^{*}$ ergibt sich aus der Bedingung

$$
\Phi_{n^{*}, n^{*}+1} \approx \hbar
$$

Mit Hilfe von Gl. (4.7) findet man

$$
n^{*} \approx \frac{\ln \hbar_{\mathrm{eff}}}{\ln \phi}
$$

wobei $\hbar_{\text {eff }}=\hbar / \Phi_{0}$ das Verhältnis des Planckschen Wirkungsquantums zum maximalen Fluss in der Kette ist. Das effektive Wirkungsquantum gibt an, wie semiklassisch sich das System verhält. Alle Volumina $\Omega_{n}$ mit $n<n^{*}$ sind durch Flüsse größer als $\hbar$ verbunden und bilden gemeinsam den Träger der chaotischen Zustände. Kettenglieder mit $n>n^{*}$ wechselwirken hingegen nur schwach mit den benachbarten Regionen und die hierarchischen Zustände sind in diesen Regionen konzentriert, solange deren Volumina groß gegenüber $\hbar$ sind. Die relative Anzahl der hierarchischen Zustände lässt sich nun leicht berechnen. Der Anteil ergibt sich aus dem Restvolumen der selbstähnlichen Kette für $n>n^{*} \mathrm{zu}$

$$
f_{\text {hier }}=\sum_{n>n^{*}} \sim \omega^{n^{*}} \sim \hbar_{\mathrm{eff}}^{1-1 / \gamma}
$$

Da $\gamma>1$ gilt, verschwindet der relative Anteil der hierarchischen Wellenfunktionen im Limes $\hbar_{\text {eff }} \rightarrow 0$, obwohl deren Gesamtzahl gemäß $\hbar_{\text {eff }}^{-1 / \gamma}$ unbeschränkt anwächst. Die Beziehung Gl. (4.11) wird eindrucksvoll von den numerischen Ergebnissen zur Standardabbildung in Abb. 4.3 unterstützt. 


\subsection{Quantenmechanische Modellierung des Phasen- raums}

Es soll nun ein Zufallsmatrizenmodell für die chaotische Dynamik im gemischten Phasenraum entwickelt werden. Vereinfachend wird wieder angenommen, dass sich die klassische Dynamik im Rahmen des Kettenmodells beschreiben lässt. Die Ableitung erfolgt in zwei Schritten. Zunächst werden die einzelnen Region $n$ durch Zufallsmatrizen beschrieben, wobei keine Wechselwirkung zwischen benachbarten Regionen bestehen soll. Dann wird gezeigt, wie man die Wechselwirkung zwischen den Regionen berücksichtigen kann.

Da die Dynamik in jeder Region $n$ als vollständig chaotisch angenommen wird, liegt eine Beschreibung durch Zufallsmatrizen nahe. Da zeitumkehrinvariante Systeme modelliert werden sollen, wird jede Region durch eine reelle hermitesche Zufallsmatrix $\mathrm{H}_{n n}$ beschrieben. Bei gegebenem $\hbar$ gibt das Volumen $\Omega_{n}$ der Region an, wie viele Zustände auf dieser Region leben und legt somit die Dimension $N_{n}$ der Zufallsmatrix fest. Die Volumina im Phasenraum werden beliebig klein. Quantenmechanisch werden allerdings nur Volumina größer als $\hbar$ aufgelöst, so dass nur endlich viele Regionen berücksichtigt werden müssen. Die Einträge der Matrix sollen gaußförmig mit der Varianz $v_{n n}^{2}$ verteilt sein. Von der Zufallsmatrizentheorie ist bekannt, dass dann die Eigenwerte $E$ nach einer Halbkreisverteilung [16]

$$
\rho(E)=\frac{2}{\pi} \sqrt{\frac{N^{2}}{4}-\frac{E^{2}}{v_{n n}^{2}}}
$$

verteilt sind. Die Varianz $v_{n n}^{2}$ legt dabei die Energieskala fest und sie wird so gewählt, dass die Eigenwerte der verschiedenen Matrizen $H_{n n}$ alle im gleichen Intervall liegen. Aus dieser Bedingung folgt, dass $N_{n} v_{n n}^{2}=$ const für alle Matrizen $\mathrm{H}_{n n}$ gelten muss. Die Matrix $H$ für eine Kette ohne Wechselwirkung zwischen den Regionen hat dann Blockdiagonalform, wobei auf der Diagonalen die Matrizen $\mathrm{H}_{n n}$ der einzelnen Regionen stehen. Speziell im Fall skalierender Volumina wie beim Kettenmodell ist es zweckmäßig, die Varianzen gemäß

$$
v_{n n}^{2}=\frac{\pi^{2}}{64} \frac{N}{\Omega \omega^{n}} \Delta^{2}
$$

zu wählen, wobei $\Omega=\Omega_{0} /(1-\omega)$ das Gesamtvolumen der Kette und $N$ die Dimension von $H$ ist. Der mittlere Niveauabstand $\Delta=h / \Omega$ hängt von der Auflösung des Phasenraums durch das Wirkungsquantum $h$ ab. Ohne Wechselwirkung erhält man eine unabhängige Überlagerung der einzelnen Subspektren und die Eigenfunktionen leben jeweils auf den einzelnen Bereichen.

Nun soll die Wechselwirkung zwischen den Bereichen diskutiert werden. Da im Kettenmodell nur benachbarte Regionen über einen bestimmten Fluss wechselwirken, hat die 


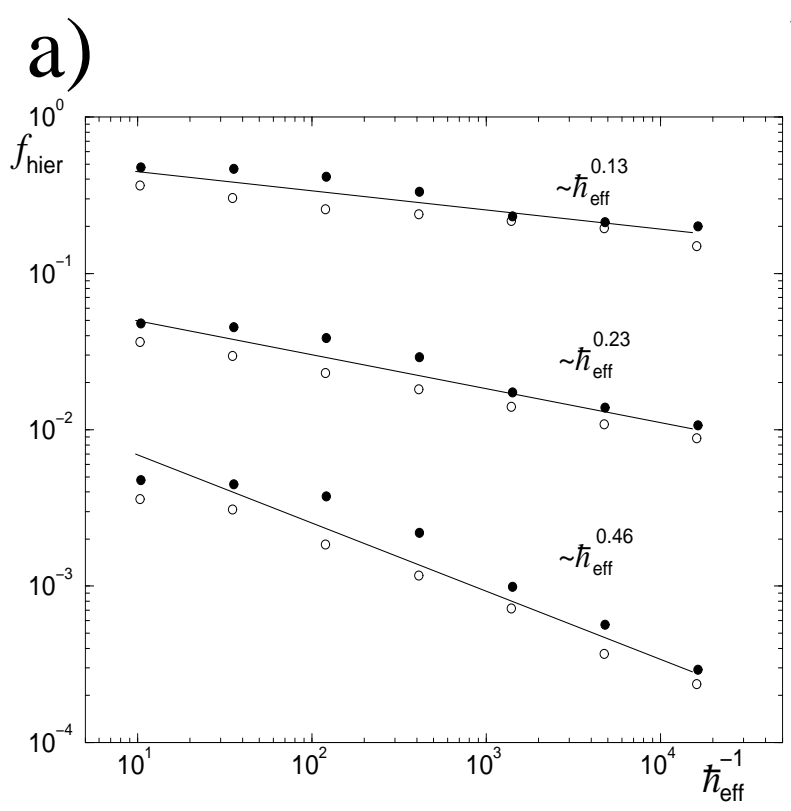

b)

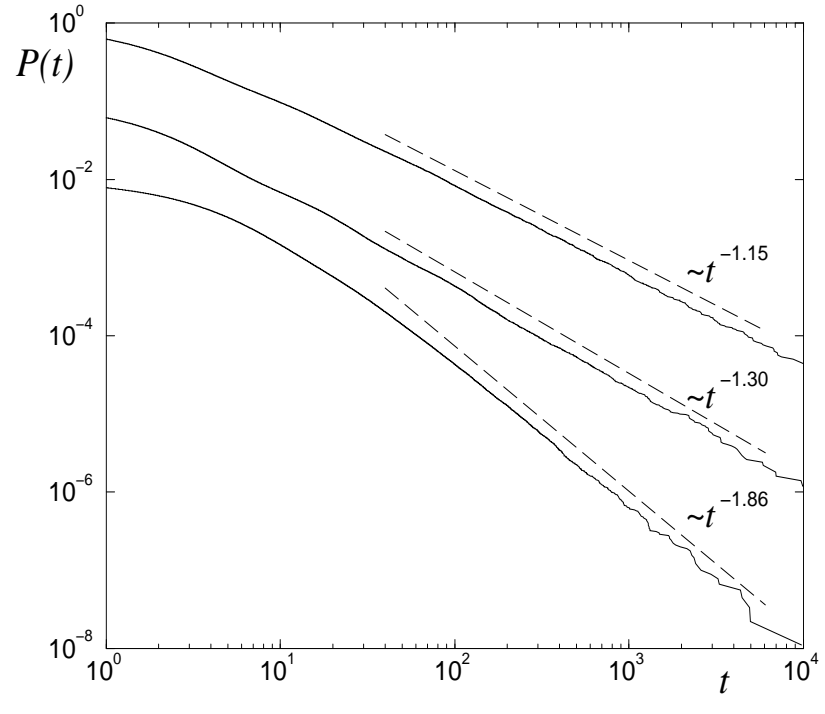

Abbildung 4.5: a) Der Anteil $f_{\text {hier }}$ der hierarchischen Eigenfunktionen für drei verschiedene Zufallsmatrixensembles $(\gamma=1.15,1.3,1.86)$ als Funktion von $1 / \hbar_{\mathrm{eff}}$. Die aus der Eigenfunktionsstatistik bestimmten Werte sind als Punkte, die aus der Eigenwertstatistik bestimmten Werte als Rauten dargestellt. Die Linien zeigen die Vorhersage nach Gl. (4.11). b) Der Zerfall der klassischen Wahrscheinlichkeit $P(t)$, der die in a) verwendeten Werte von $\gamma$ bestätigt.

Zufallsmatrix mit Wechselwirkung die Gestalt

$$
H=\left(\begin{array}{cccc}
\mathrm{H}_{00} & \mathrm{~W}_{01} & & \\
\mathrm{~W}_{10} & \mathrm{H}_{11} & \mathrm{~W}_{12} & \\
& \mathrm{~W}_{21} & \mathrm{H}_{22} & \ddots \\
& & \ddots & \ddots
\end{array}\right)
$$

Die Matrizen $W_{n n+1}$ beschreiben dabei die Wechselwirkung zwischen den Blöcken $n$ und $n+1$. Damit $H$ hermitesch ist, muss $W_{n+1 n}=W_{n n+1}^{T}$ gelten. Die einzelnen Elemente der Koppelungsmatrizen sollen wieder gaußsch verteilt sein. Die Koppelungsstärke wird durch die Varianz $v_{n n+1}^{2}$ festgelegt. In Ref. [73] wurde allgemein gezeigt, dass die Varianz gemäß

$$
v_{n n+1}^{2}=\frac{\Delta^{2}}{4 \pi f_{n} f_{n+1}} \frac{\Phi_{n, n+1}}{h}
$$

vom klassischen Fluss $\Phi_{n, n+1}$ zwischen den Regionen $n$ und $n+1$ abhängt. Dabei ist $f_{n}=\Omega_{n} / \Omega$ der relative Anteil der Region $n$ am gesamten Phasenraum. Man erkennt, dass der effektive Koppelungsparameter der Fluss in Einheiten von $\hbar$ ist. Speziell für das Kettenmodell erhält man

$$
v_{n n+1}^{2}=\frac{\Delta^{2}}{4 \pi f_{0}^{2}}\left(\frac{\phi}{\omega}\right)^{n} \frac{1}{\hbar_{\mathrm{eff}}}
$$


Die Gleichungen 4.13 und 4.16 stellen die Verbindung zwischen den klassischen Eigenschaften des Phasenraums und der quantenmechanischen Realisierung dar.

Durch Wahl der Skalierungsparameter $\omega$ und $\phi$ kann der klassische Exponent $\gamma$ nach Gl. (4.8) eingestellt werden. Im Matrix-Modell können nun mit den gleichen Analysemethoden, wie sie auch zur Untersuchung des gekickten Rotators verwendet wurden, die Eigenschaften der hierarchischen Zustände untersucht werden. Dazu wird bei festen Parametern über mehrere Realisierungen der Zufallsmatrix gemittelt. In Abb. 4.5 sind die so berechneten Resultate zusammen mit dem klassischen Zerfall $P_{\mathrm{cl}}(t)$ des Kettenmodells gezeigt. Die numerischen Daten stimmen über drei Größenordnungen mit der Vorhersage Gl. (4.11) überein.

\subsection{Zusammenfassung und Ausblick}

In diesem Kapitel wurde die Existenz einer neuen Klasse von Eigenfunktionen in gemischten Systemen gezeigt. Diese hierarchischen Eigenfunktionen sind eng verknüpft mit der klassischen Struktur des Phasenraums. Sie haben als Träger einen Teil der chaotischen invarianten Menge und werden durch die Flussbarriere von den chaotischen Eigenfunktionen getrennt. Ihr Einfluss auf die statistischen Eigenschaften des Spektrums und der Eigenfunktionen wurde diskutiert. Eine quantenmechanische Behandlung der klassischen Dynamik erlaubte es, das Skalierungsverhalten des relativen Anteils der hierarchischen Eigenfunktionen mit $\hbar_{\text {eff }}$ zu berechnen. Diese Vorhersagen wurden am gekickten Rotator und einem Zufallsmatrizenmodell numerisch verifiziert, dabei erwies sich die Flussbarriere als anschauliches und fundamentales Konzept.

Die Ergebnisse dieses Kapitels sollten die Grundlage für die Suche nach weiteren Quantensignaturen gemischter Systeme bilden. Da zumindest im semiklassischen Limes die Quantenmechanik der Klassik folgt, müssen die typischen Eigenschaften der chaotische Dynamik gemischter Systeme in Spektrum und Eigenfunktionen kodiert sein. Im Gegensatz zur Zufallsmatrizentheorie, die Spektrum und Eigenfunktionen getrennt behandelt, sind hier Korrelationen wichtig. Diese Korrelationen gilt es zu entschlüsseln. Dabei kann die Rückkehrwahrscheinlichkeit $C(t)=|\langle\psi(0) \mid \psi(t)\rangle|^{2}$ den Weg weisen, da diese Größe über eine Fouriertransformation mit dem Spektralmaß bzw. der lokalen Zustandsdichte verbunden ist. Ein weiterer vielversprechender Weg ist zu untersuchen, wie ein gemischtes System auf Parametervariation reagiert. So kann man z.B. die Veränderung des Spektrums bei Variation einer Blochphase studieren, denn wie Akkermans und Montambaux aufzeigten, besteht ein Zusammenhang zwischen der Krümmung der Eigenenergien und dem Leitwert [74]. Und für den Leitwert als Funktion eines Parameters sind Quantensignaturen des gemischten Phasenraums bekannt, wie im folgenden Kapitel ausführlich dargelegt wird. 


\section{Kapitel 5}

\section{Leitwertfluktuationen}

Universelle Leitwertfluktuationen, wie sie an ungeordneten Quantenpunkten und ballistischen Billards beobachtet werden [24-27], sind ein Paradigma chaotischer Streuprozesse [28-31]. Die Dynamik typischer Billards ist jedoch nicht vollständig chaotisch, sondern zeichnet sich durch einen gemischten Phasenraum aus. Eine semiklassische Analyse der Dynamik führte zur Vorhersage fraktaler Leitwertfluktuationen [34], die auch experimentell [75-79] und numerisch [80] bestätigt wurden. Berechnungen des Leitwerts am Cosinus-Billard mit gemischtem Phasenraum zeigen jedoch keine fraktalen Eigenschaften, sondern viele isolierte Resonanzen [35]. Dieser Widerspruch wird in diesem Kapitel aufgelöst und eine allgemeine Theorie zu Leitwertfluktuationen in gemischten Systemen wird aufgestellt $[37,39]$.

\subsection{Transport mesoskopischer Systeme}

\subsubsection{Quantenpunkte}

Eine typische Methode zur Herstellung von Quantenpunkten ist in Abb.5.1 dargestellt. Auf dem Halbleiter GaAs lässt man mehrere Lagen AlGaAs aufwachsen. Elektronen sammeln sich an der Grenzfläche der beiden Halbleiter und es bildet sich ein zweidimensionales Elektronengas (2DEG). Mit Hilfe von lithographischen Verfahren lassen sich metallische Kontakte auf die Heterostruktur aufbringen, die es ermöglichen, die Bewegung der Elektronen auf bestimmte Bereiche zu beschränken. Der Quantenpunkt koppelt an das 2DEG über zwei Öffnungen (source und drain). Ein Spannungsunterschied $V_{\text {sd }}$ zwischen den beiden Öffnungen erzeugt einen Strom $I$. So kann der Leitwert $g=I / V_{\text {sd }}$ des Quantenpunkts bestimmt werden. Die physikalischen Eigenschaften des Quantenpunkts hängen von verschiedenen Parametern ab: Zunächst wäre da die Abmessung des Quantenpunkts 

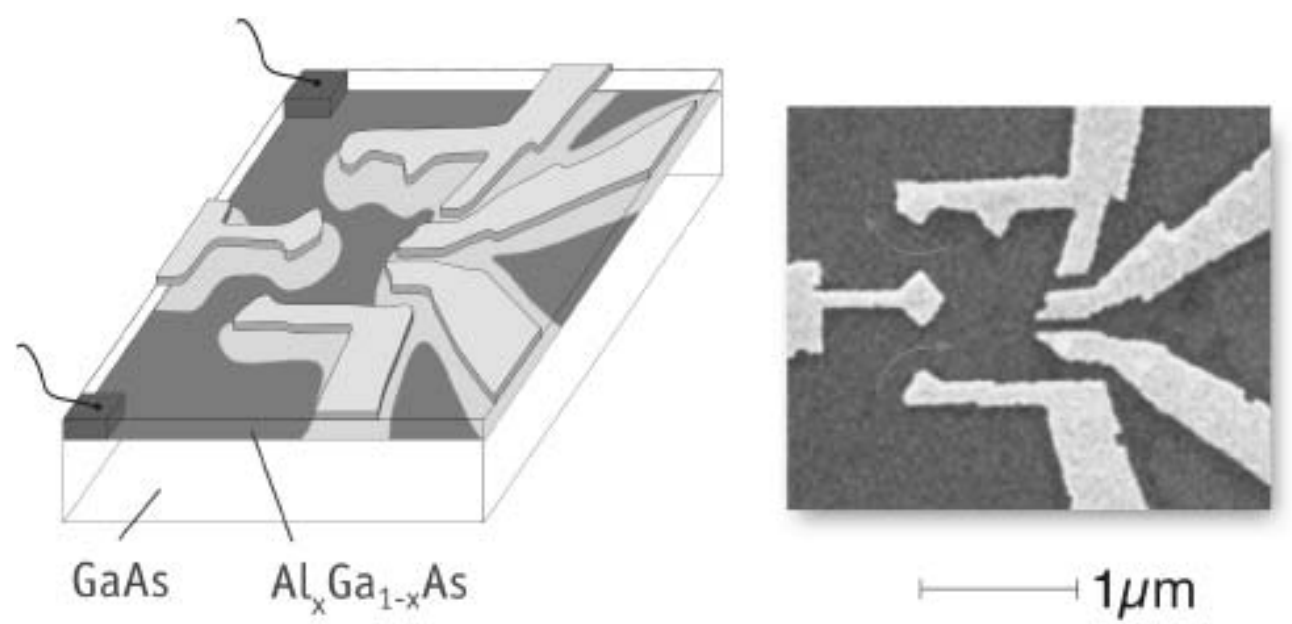

\begin{abstract}
Abbildung 5.1: Schematischer Aufbau einer Halbleiter Heterostruktur [81]. An der Grenzfläche der beiden Halbleiter GaAs und AlGaAs bildet sich ein 2DEG aus, welches durch Kontakte auf der Heterostruktur auf bestimmte Regionen beschränkt werden kann. Rechts ist eine elektronenmikroskopische Aufnahme eines Quantenpunkts gezeigt.
\end{abstract}

$L \sim \sqrt{A}$ zu nennen, wobei $A$ die Fläche des Quantenpunkts ist. Typische Werte liegen zwischen $1-2 \mu \mathrm{m}$. Bei niedrigen Temperaturen tragen nur Elektronen mit Energien nahe der Fermi-Energie zum Transport bei. Die Eigenschaften dieser Elektronen werden durch die Fermi-Wellenlänge $\lambda_{F}=2 \pi / k_{F}$ bestimmt, die für typische Elektronendichten bei ca. $40 \mathrm{~nm}$ liegt. Der Impuls eines Elektrons kann sich durch Wechselwirkung mit Störstellen, Phononen oder anderen Elektronen ändern. Die mittlere freie Weglänge $l_{c}$ ist die Strecke, die ein Elektron zurücklegt, ohne dass der initiale Impuls zerstört wird. Bei tiefen Temperaturen und geringer Störstellendichte kann die mittlere freie Weglänge ein Vielfaches der Abmessung des Quantenpunkts betragen $\left(l_{c} \approx 30 \mu m\right)$. Schließlich muss noch die Phasenkohärenzlänge erwähnt werden. Es ist die typische Länge, die ein Elektron zurücklegt, ohne seine Phaseninformationen zu verlieren. Bei inelastischen Streuprozessen wie z.B. mit Phononen, anderen Elektronen oder Störstellen mit internen Freiheitsgraden gehen Phaseninformationen verloren. Der Einfluss dieser Größen soll nun diskutiert werden.

\title{
5.1.2 Landauer Formel
}

Bei der klassischen Beschreibung des Elektronentransports wird angenommen, dass die Streuung der Elektronen an Störstellen die Bewegung dominiert $\left(l_{c}<L\right)$. Durch diese Streuprozesse verlieren die Elektronen die Phaseninformationen $\left(L_{\varphi} \approx l_{c}\right)$ und die Elektronenbewegung lässt sich daher durch einen Diffusionsprozess beschreiben, was zum Ohm'schen Gesetz für die Leitfähigkeit führt. Für mesoskopische Systeme $\left(L_{\varphi} \geq L\right)$ spielen phasenkohärente Effekte hingegen eine wichtige Rolle. Eine Möglichkeit, diese Phaseneffekte zu berücksichtigen, wurde von Landauer [82,83] und Imry [84] aufgezeigt. Der Strom 
durch eine Probe wird dabei verknüpft mit der Transmissionswahrscheinlichkeit $t$ eines Elektron durch die Probe. Dies liefert die Landauer Leitfähigkeit

$$
g=\frac{2 e^{2}}{h} M t
$$

wobei $M$ die Anzahl der Moden angibt. Die Übergangswahrscheinlichkeit von einem Kanal in einen anderen Kanal ist durch die $S$-Matrix gegeben. Für zwei Kanäle hat die $S$-Matrix die Form

$$
\mathbf{S}=\left(\begin{array}{ll}
R_{11} & T_{21} \\
T_{12} & R_{22}
\end{array}\right)
$$

Die Matrix $T_{12}$ bzw. $T_{21}$ beschreibt die Transmission durch den Quantenpunkt. Drückt man die Transmissionswahrscheinlichkeit $t$ durch die $S$-Matrix aus, so erhält man

$$
g=\frac{2 e^{2}}{h} \operatorname{tr} T T^{\dagger}
$$

dabei wurde vereinfachend angenommen, dass das Streuproblem symmetrisch ist. Gleichung (5.3) ist der Ausgangspunkt für die theoretische Beschreibung von Leitwerteigenschaften mesoskopischer Systeme.

\subsection{Leitwertfluktuationen}

Ein zentrales Phänomen mesoskopischer Systeme sind Leitwertfluktuationen. Dabei wird die Veränderung des Leitwerts in Abhängigkeit eines externen Parameters, wie z.B. der magnetischen Feldstärke oder der Energie, untersucht.

\subsubsection{Universelle Leitwertfluktuationen}

Dies geschah zunächst für ungeordnete Systeme, die sich dadurch auszeichnen, dass die mittlere freie Weglänge zwischen elastischen Stößen kleiner als die Abmessung des Quantenpunkts ist $\left(l_{c}<L\right)$. Für solche Systeme wurden universelle Leitwertfluktuationen (ULF) beobachtet [24-27]. Dies sind reproduzierbare Fluktuationen der Größenordnung $e^{2} / h$ um den mittleren Leitwert. Sie sind unabhängig von Materialeigenschaften und dem mittleren Leitwert. Eine Verbindung zwischen ULF und der Theorie der Zufallsmatrizen konnte hergestellt werden [28-30] und in diesem Sinn sind ULF ein Paradigma chaotischer Streuprozesse.

Fortschritte in der Herstellung von Halbleiter-Heterostrukturen erlauben es, Quantenpunkte mit einer sehr geringen Störstellenkonzentration $\left(l_{c}>L\right)$ herzustellen. In solchen Heterostrukturen bewegen sich Elektronen ballistisch, unterbrochen durch Reflektionen an den 
Wänden. Solche Quantenpunkte werden im Folgenden als Billards bezeichnet. Während für ungeordnete Systeme die genaue Form des Quantenpunkts unerheblich ist, spielt sie hier eine entscheidende Rolle. Mit Hilfe von semiklassischen Methoden kann eine Verbindung zwischen der klassischen Dynamik des Billards und dem Leitwert erstellt werden. Die semiklassische Näherung für die $S$-Matrix lautet $[85,86]$

$$
S_{i j}(E)=\sum_{s} \sqrt{P_{j \rightarrow i}^{(s)}} e^{\frac{i}{\hbar} S^{(s)}(E)-\frac{i \pi}{2} \nu^{(s)}}
$$

Die Summe erstreckt sich über alle Trajektorien und $P_{j \rightarrow i}^{(s)}$ ist die klassische Wahrscheinlichkeit von Kanal $j$ zum Kanal $i$ zu gelangen. Die Phaseninformationen entlang eines Pfades werden durch die Wirkung $S(E)$ und den Maslov-Index $\nu$ berücksichtigt. Unter der Annahme chaotischer Dynamik im Billard, charakterisiert durch einen exponentiellen Zerfall $P_{\mathrm{cl}}(t) \sim \exp (-\alpha t)$, konnten Blümel und Smilansky zeigen, dass die semiklassische Analyse zu ULF führt [87]. Die klassische Zerfallsrate findet sich in der Korrelationsfunktion

$$
C(\Delta E)=\langle g(E) g(E+\Delta E)\rangle_{E}
$$

für kleine $\Delta E$ wieder

$$
C(\Delta E)=\frac{C_{0}}{1+\left(\frac{\Delta E}{\alpha \hbar}\right)^{2}} .
$$

Diese Lorenz-Form wurde experimentell für vollständig chaotische Billards beobachtet [88].

\subsubsection{Fraktale Leitwertfluktuationen}

Typische Billards sind jedoch weder regulär noch chaotisch, sondern besitzen einen gemischten Phasenraum. Lai et. al. haben gezeigt, dass das algebraische Hängenbleiben an den Inseln $P_{\mathrm{cl}}(t) \sim t^{-\gamma}$ im Vergleich zu vollständig chaotischen Systemen zu Fluktuationen der $S$-Matrix auf kleineren Skalen führt [89]. Dies spiegelt sich in der Autokorrelationsfunktion wieder, die im Ursprung nicht mehr die Form einer Lorenzkurve aufweist, sondern gemäß

$$
C(\Delta E)=C_{0}+C_{1}(\Delta E / \hbar)^{\gamma}
$$

abfällt. Dieses Verhalten wurde auch numerisch für eine Kette von Potentialmulden mit gemischtem Phasenraum verifiziert [89]. Genauer bleibt festzuhalten, dass es für die Herleitung von Gl. (5.7) notwendig ist, dass die zur Parametervariation konjugierte Größe algebraisch abfällt. Bei einer Variation der Energie ist dies genau die Verweildauer im Billard. Wird die magnetische Feldstärke variiert, so muss die Verteilung der Flächen, die von Orbits umschlossen werden, algebraisch abfallen. Ketzmerick hat gezeigt, dass unter diesen Voraussetzungen die Leitwertkurve die statistischen Eigenschaften einer fraktionalen Brown'schen Bewegung besitzt [34]. Solche Kurven werden durch eine fraktale Dimension 

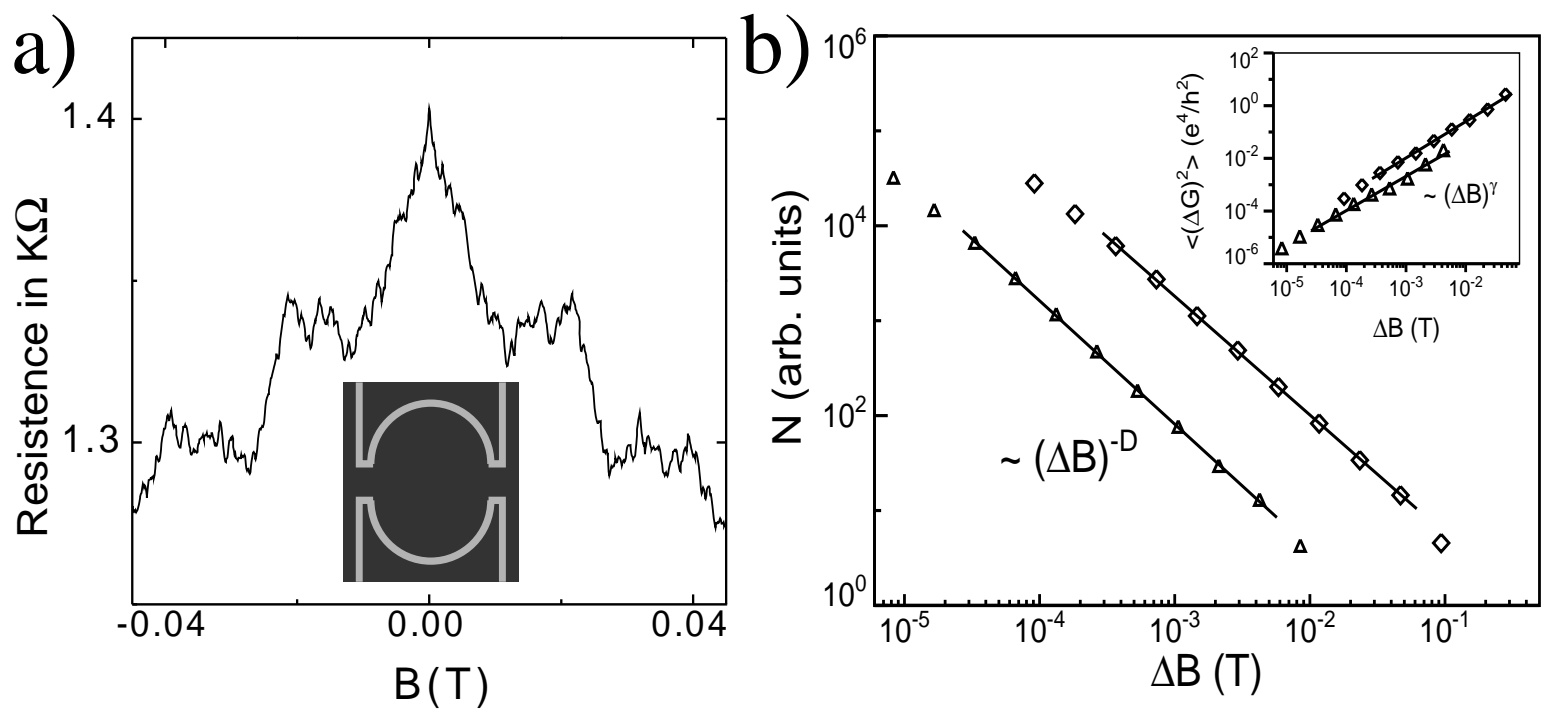

Abbildung 5.2: a) Fraktale Leitwertfluktuationen, gemessen am Stadion-Billard (s. Inset) [76]. Deutlich sind Fluktuationen auf verschiedenen Skalen zu sehen. b) Die fraktale Analyse des Leitwerts aus a) zeigt skalierendes Verhalten über zwei Größenordnungen. Das Inset zeigt das entsprechende Potenzgesetz in der Varianz der Leitwertinkremente.

$D$ charakterisiert [90]. Daher stammt der Name fraktale Leitwertfluktuationen (FLF). Die fraktale Dimension ist gegeben durch [34]

$$
D=2-\gamma / 2
$$

wenn $1<\gamma \leq 2$ gilt. Ist $\gamma>2$, so ist die fraktale Dimension unabhängig von $\gamma$ stets $D=1$. Abbildung 5.2 zeigt eine experimentell bestimmte Leitwertkurve [76]. Deutlich sind Fluktuationen auf verschiedenen Skalen erkennbar und die Analyse der statistischen Eigenschaften bestätigt, dass es sich hierbei um eine fraktale Kurve handelt. Fraktale Leitwertfluktuationen wurden für verschiedene Systeme experimentell beobachtet [75-79]. Jedoch ist es experimentell nicht möglich, die Abhängigkeit der fraktalen Dimension vom klassischen Exponenten $\gamma$ nach Gl. (5.8) zu verifizieren, da $\gamma$ experimentell nicht bestimmbar ist. Numerische Untersuchungen des Leitwerts, mit deren Hilfe man die Beziehung Gl. (5.8) überprüfen könnte, gestalten sich als schwierig, da die Berechnung der Streumatrix für semiklassische Systeme sehr aufwendig ist. Eine Möglichkeit, diese Schwierigkeiten zu umgehen, haben Casati et. al. vorgeschlagen [80]. Anstatt den Leitwert zu berechnen, untersuchten sie, wie sich die quantenmechanische Aufenthaltswahrscheinlichkeit für große Zeiten bei Parameteränderung verhält. Die beobachteten fraktalen Fluktuationen können semiklassisch in Analogie zu den FLF erklärt werden. Da für das untersuchte System auch der klassische Exponent $\gamma$ bekannt ist, konnte der Zusammenhang zwischen $\gamma$ und der fraktalen Dimension in Gl. (5.8) verifiziert werden. 


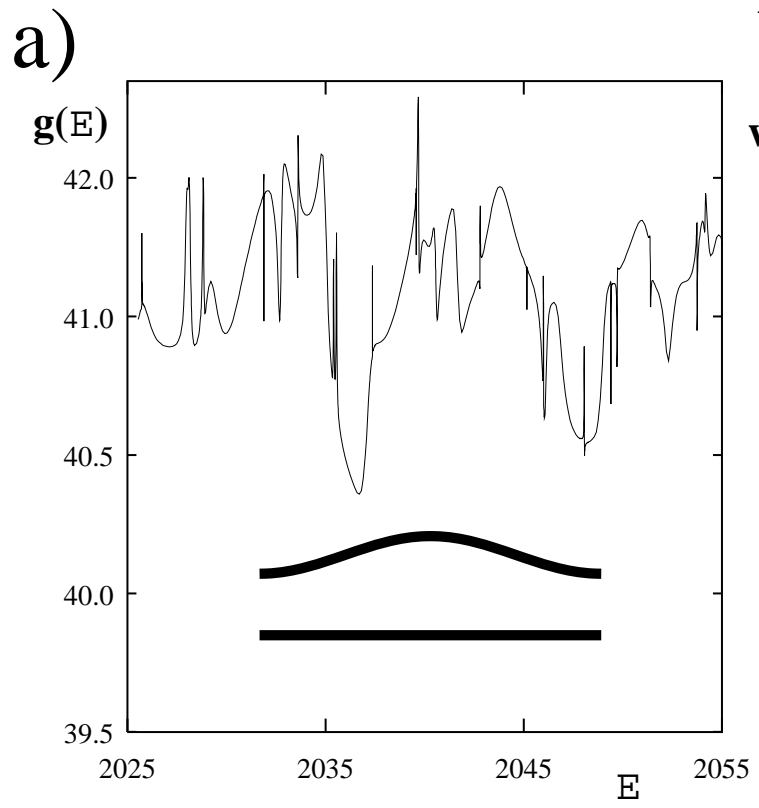

b)

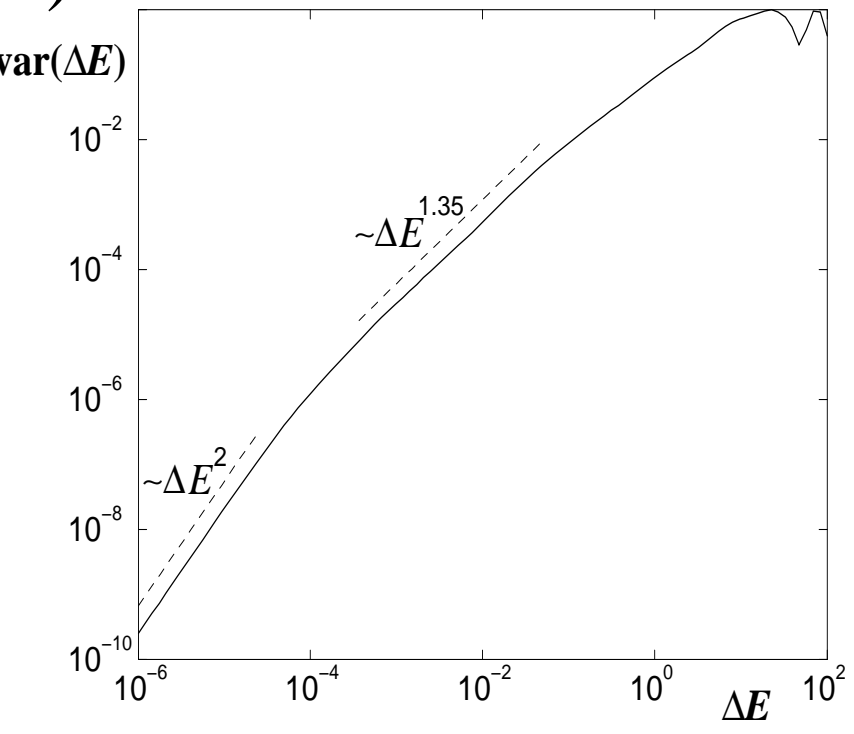

Abbildung 5.3: a) Berechneter Leitwert für das Cosinus Billard (Sketch s. Inset) entnommen aus Ref. [35]. Auf einer glatten Kurve sind viele isolierte Resonanzen zu sehen. b) Varianz der Leitwertinkremente für die Kurve in a). Auf ganz kleinen Skalen, $\Delta E<10^{-4}$, ist die Kurve glatt und man findet einen quadratischen Anstieg in $\operatorname{var}(\Delta E)$. Auf Skalen größer als der mittlere Niveauabstand gilt $\operatorname{var}(\Delta E)=$ const.. Dazwischen gibt es einen Bereich, der ein Potenzgesetz aufweist, dessen Exponent mit dem klassischen Zerfallsexponenten, $\gamma=1.35$ für die gewählten Parameter, übereinstimmt.

\subsubsection{Isolierte Resonanzen}

Ein gänzlich anderes Bild für den Leitwert zeigt eine neue Arbeit von Huckestein et. al. [35], in der der Leitwert für das Cosinus-Billard numerisch untersucht wurde. Der Leitwert als Funktion der Energie zeichnet sich durch einen glatten, langsam variierenden Kurvenverlauf aus, der durch viele isolierte Resonanzen unterbrochen wird(Abb. 5.3). Eine genauere Analyse der Kurve zeigt, dass die Varianz der Leitwertinkremente

$$
\operatorname{var}(\Delta E) \equiv\left\langle(g(E+\Delta E)-g(E))^{2}\right\rangle_{E} \quad,
$$

für kleine $\Delta E$ ein algebraisches Verhalten $\operatorname{var}(\Delta E) \sim|\Delta E|^{\beta}$ aufweist. Es wurde spekuliert, dass der Exponent $\beta$ gleich dem klassischen Zerfallsexponenten $\gamma$ sei. Dies widerspricht jedoch der physikalischen Intuition, da das gefundene Potenzgesetz für Abstände gefunden wurde, die kleiner als der mittlere Niveauabstand $\Delta$ sind, und somit eine rein quantenmechanische Ursache haben muss. Es liegt die Vermutung nahe, dass die isolierten Resonanzen von regulären Zuständen auf den Inseln herrühren. Wäre dies so, dann müssten ungefähr so viele isolierte Resonanzen wie reguläre Zustände vorliegen, wobei die Anzahl der regulären Zustände gut durch die Größe der Inseln im Phasenraum abgeschätzt werden kann. Man 
a)

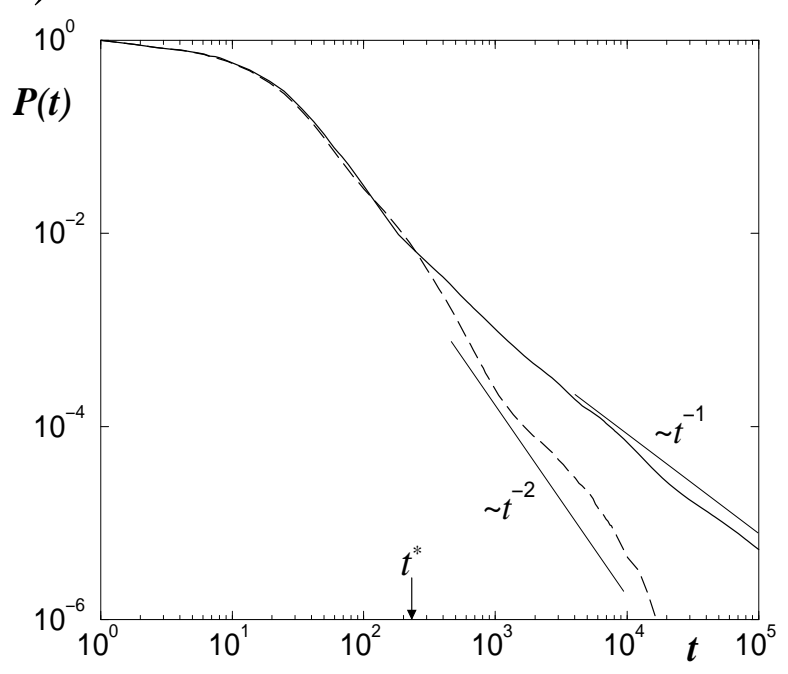

b)

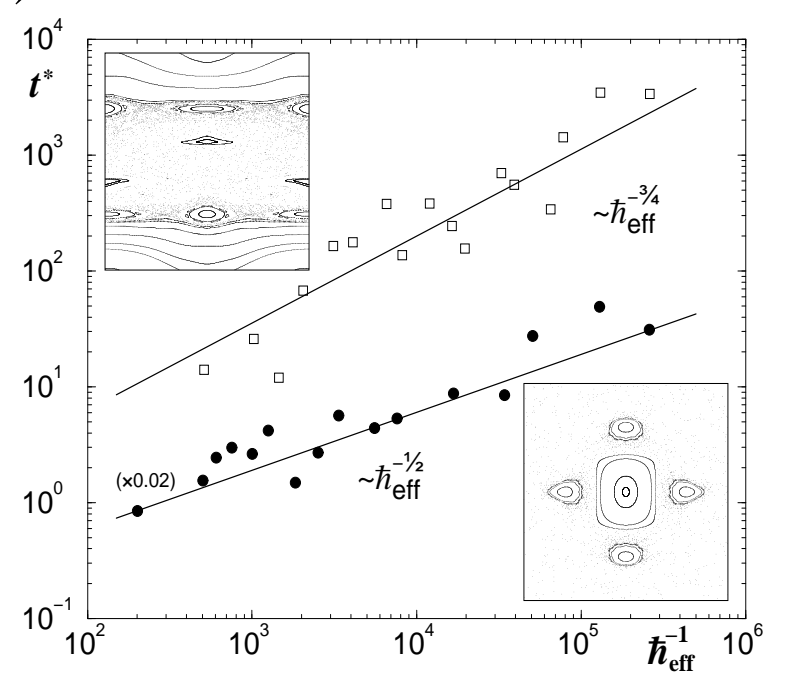

Abbildung 5.4: a) Gezeigt sind der klassische Zerfall $P_{\mathrm{cl}}(t)$ (gestrichelt) zusammen mit dem quantenmechanischen $P_{\mathrm{qm}}(t)$ für den gekickten Rotator bei $K=2.5$. Bis zur Zeit $t^{*}$ folgt die Quantenmechanik dem klassischen Zerfall. b) Skalierungsverhalten der Zeitskala $t^{*}$ für die Separatrix- (Quadrate) und die Standardabbildung (volle Punkte). Die Linien geben das vorhergesagte Skalierungsverhalten nach Gl. (5.11) an. Dabei ist der klassische Exponent für die Separatrixabbildung 4/3. Die Daten der Standardabbildung entstammen Ref. [91] und folgen näherungsweise dem dort vorhergesagten Verhalten $t^{*} \sim \sqrt{\hbar_{\text {eff }}}$, das man mit $\gamma \approx 1.9$ auch mit Gl. (5.11) erhält. Die Insets zeigen die Phasenraumstrukturen (symmetrisiert) für die beiden Abbildungen.

findet jedoch, dass es mehr als doppelt so viele isolierte Resonanzen wie reguläre Zustände gibt. Dies soll nun aufgelöst werden.

\subsection{Fraktal oder isoliert?}

Es soll nun der Ursprung der isolierten Resonanzen erklärt werden. Die Ableitung der FLF geht davon aus, dass die quantenmechanische Aufenthaltswahrscheinlichkeit $P_{\mathrm{qm}}(t)$ im Billard dem klassischen Potenzgesetz folgt. Numerisch findet man jedoch (s. Abb. 5.4a), dass $P_{\mathrm{qm}}(t)$ dem klassischen Zerfall nur für endliche Zeiten folgt. Daher soll nun untersucht werden, wie lange die Quantenmechanik die klassische Dynamik nachahmt. Im strickten Sinn ist dies die Ehrenfest Zeit $\tau_{\mathrm{E}}$. Es ist die Zeit, bis zu der ein Wellenpaket mit minimaler Unschärfe dem klassischen Orbit folgt. Auf Grund der exponentiellen Separation chaotischer Trajektorien, charakterisiert durch einen Lyapunov-Exponente $\lambda>0$, ist diese Zeit logrithmisch klein $\tau_{\mathrm{E}} \sim 1 /(2 \lambda) \ln (1 / \hbar)$ [46]. Ist man jedoch an der mittleren zeitlichen Ent- 
wicklung eines Operators interessiert, so kann diese der entsprechenden klassischen Größe für erheblich längere Zeiten folgen. Für offene Quantensysteme liefert die Flussbarriere an der Stelle $n^{*}$ eine wichtige Zeitskala. Im Rahmen des Kettenmodells (Abschnitt 2.4.3) ist sie durch

$$
t^{*}=\Omega_{n^{*}+1} / \Phi_{n^{*}, n^{*}+1}
$$

gegeben. Für Zeiten $t>t^{*}$ erkunden die klassischen Trajektorien Bereiche hinter der Flussbarriere. Da die Quantendynamik die klassischen Flüsse $\Phi_{n, n+1}$ mit $n>n^{*}$ nicht nachahmen kann, muss für Zeiten $t>t^{*}$ die Quantenmechanik von der Klassik abweichen. Das Skalierungsverhalten des Kettenmodells führt zu der Beziehung

$$
t^{*}=\tau_{\mathrm{H}} \hbar_{\mathrm{eff}}^{1-1 / \gamma},
$$

wobei $\tau_{\mathrm{H}}=h / \Delta$ die Heisenbergzeit und $\Delta$ der mittlere Niveauabstand ist. Um die Vorhersage zu überprüfen, wurde die Separatrix- und Standardabbildung genauer untersucht. Dazu wird zu verschiedenen $\hbar_{\text {eff }}$ eine Anfangsverteilung, die der klassischen Verteilung entspricht, über viele Zeitschritte $\left(10^{5}\right)$ iteriert. Nach jeder Iteration erfolgt eine Projektion des Wellenpakets auf einen Teil der Impulszustände. Bei der Separatrixabbildung werden alle Impulse $p<0$ abgeschnitten, bei der Standardabbildung werden nur Impulse im Fenster $-\pi<p<\pi$ weiter berücksichtigt (die Phasenraumstrukturen sind Abb. 5.4b gezeigt). Der klassische Zerfall für die Separatrixabbildung zeigt über viele Größenordnungen ein Potenzgesetz mit dem Exponenten $\gamma=4 / 3$. Dies passt gut zum beobachteten Skalierungsverhalten $t^{*} \sim \hbar_{\text {eff }}^{-3 / 4}$, wobei man berücksichtigen muss, dass für eindimensionale gekickte Systeme $\tau_{\mathrm{H}} \sim \hbar_{\text {eff }}^{-1}$ gilt (der mittlere Niveauabstand skaliert wie $\Delta \sim \hbar_{\text {eff }}$ ). Die Daten der Standardabbildung entstammen der Ref. [91]. Dort wurde für Systeme mit gemischten Phasenraum ganz allgemein ein Skalierungsverhalten $t^{*} \sim \hbar_{\mathrm{eff}}^{-0.5}$ vorhergesagt, das man auch schon in vollständig chaotischen Systemen gefunden hat [92,93]. Für den untersuchten Parameter $(K=2.5)$ ist jedoch der klassische Zerfallsexponent gerade $\gamma=1.9$, der gemäß Gleichung 5.11 somit zufällig das geforderte Verhalten $t^{*} \sim \hbar_{\mathrm{eff}}^{-0.5}$ liefert. Beide Datensätze bestätigen sehr gut das Skalierungsverhalten Gl. (5.11), welches die spezifische Natur des gemischten Phasenraums berücksichtigt.

Die zur Zeitskala $t^{*}$ korrespondierende Energieskala

$$
\Delta E^{*}=\Delta \hbar_{\mathrm{eff}}^{1 / \gamma-1}
$$

bestimmt das Verhalten der Leitwertkurve. Fluktuationen unterhalb der Energieskala $\Delta E^{*}$ werden von den hierarchischen Zuständen, die hinter der Flussbarriere leben, verursacht. Diese Zustände koppeln nur schwach nach außen und haben daher eine sehr kleine Resonanzbreite $\Gamma$, was zu isolierten Resonanzen der Breite $\Delta E \approx \Gamma$ in der Leitwertkurve $G(E)$ führt. Auf Skalen $\Delta E>\Delta E^{*}$ findet man die FLF. Diese werden durch die Zustände vor der Flussbarriere erzeugt, die auch dafür verantwortlich sind, dass die Quantenmechanik dem klassischen Zerfall bis zur Zeit $t^{*}$ folgt.

Da die Regionen hinter der Flussbarriere nur schwach an die vorderen Regionen koppeln, kann man jeder Eigenenergie der abgeschlossenen Regionen eindeutig einen Pol und damit 
eine Resonanzbreite des Streusystems zuordnen. Die Verteilung der Resonanzbreiten $p(\Gamma)$ der Regionen hinter der Flussbarriere kann daher störungstheoretisch berechnet werden. Eine typische Resonanzbreite $\Gamma_{n}$ eines Zustandes, der in der $n$-ten Region, $n>n^{*}+$ 1, lokalisiert ist, erhält man als Produkt der individuellen Koppelungen aller Regionen zwischen der Flussbarriere und der $n$-ten Region. Dies liefert

$$
\frac{\Gamma_{n}}{\Gamma^{*}}=\prod_{j=n^{*}+1}^{n-1} \frac{\Phi_{j, j+1}}{\hbar^{d-1}}=\prod_{j=n^{*}+1}^{n-1} \varphi^{j-n^{*}} \approx \varphi^{\left(n-n^{*}\right)^{2} / 2}
$$

wobei $\Gamma^{*}=h / t^{*}$ die Resonanzbreite an der Flussbarriere ist und zur Vereinfachung $n-$ $n^{*}>>1$ angenommen wurde. Verwendet man die integrierte Verteilung

$$
P_{\text {int }}\left(\Gamma_{n} / \Gamma^{*}\right)=\int_{0}^{\Gamma_{n} / \Gamma^{*}} p(\Gamma) d \Gamma \sim \sum_{j=n}^{\infty} \Omega_{j} \sim \omega^{n}
$$

so findet man schließlich

$$
p(\Gamma) \sim \frac{1}{\Gamma} \cdot \frac{\exp \left(\ln \omega \sqrt{2 \ln \left(\Gamma / \Gamma^{*}\right) / \ln \varphi}\right)}{\sqrt{-\ln \Gamma / \Gamma^{*}}}
$$

auf Skalen $\Gamma<\Gamma^{*}$. Asymptotisch konvergiert diese Verteilung gegen $p(\Gamma) \sim 1 / \Gamma$ für kleine $\Gamma$. Jedoch kann es - je nach Parameterwahl - einen breiten Übergangsbereich über mehrere Größenordnungen geben, ehe das asymptotische Verhalten sichtbar wird.

\subsection{Graphenmodell für den gemischten Phasenraum}

Um die obigen Vorhersagen zu testen, sollen nun die Streueigenschaften eines quantenmechanischen Pendants des klassischen Kettenmodells (Abschnitt 2.4.3) untersucht werden. Dazu werden Quantengraphen verwendet, da diese eine natürliche Darstellung durch Streumatrizen besitzen.

\subsubsection{Quantengraphen}

Kottos und Smilansky haben gezeigt, dass spektrale und Streueigenschaften vollständig vernetzter Quantengraphen der Zufallsmatrizentheorie gehorchen [94,95]. Quantengraphen bestechen durch ihre konzeptionelle Transparenz und einfache numerische Implementierbarkeit. Darüber hinaus kann die zur Quantenmechanik korrespondierende klassische Dynamik auf Graphen eingeführt werden, was Zufallsmatrizenmodelle nicht gestatten. 


\section{Graphen}

Ein Graph $\mathcal{G}$ besteht aus einer Menge von Knoten (Vertices) $\mathcal{V}=1, \ldots, n_{\mathcal{V}}$, die durch Kanten (Bonds) $B=1, \ldots, n_{\mathcal{B}}$ verbunden sind. Die Knoten werden im Folgenden zumeist mit $v$, die Kanten mit $b$ bezeichnet. Die Valenz $\mathrm{d}(v)$ eines Knotens $v$ gibt die Anzahl der Kanten an, die mit diesem Knoten verbunden sind. Jeder Kante des Graphen wird eine Länge $L_{b}>0$ zugeordnet und die Summe aller Kantenlängen liefert die Gesamtlänge $L$ des Graphen. Ein Orbit $O$ auf dem Graphen ist eine Abfolge von Kanten, so dass zwei aufeinander folgende Kanten mit dem selben Knoten verbunden sind. Die Länge des Orbits $L_{O}$ ist gegeben durch die Summe der einzelnen Kantenlängen multipliziert mit der Anzahl, wie oft sie durchlaufen werden. Ein Orbit heißt geschlossen, wenn der Knoten, an dem der Orbit endet, auch der Startknoten ist. Die $n_{\mathcal{B}}$ Kanten können jeweils in zwei Richtungen durchlaufen werden. Ist die Richtung, mit der eine Kante durchlaufen wird, wichtig, so spricht man von gerichteten Kanten. Die $2 n_{\mathcal{B}}$ gerichteten Kanten seien mit $d$ bezeichnet. Jede gerichtete Kante hat einen Anfangs- und Endpunkt. Man sagt, die Kante $d^{\prime}$ folgt der Kante $d$, wenn $d^{\prime}$ an dem Knoten beginnt, an dem $d$ endet. Die Topologie des Graphen lässt sich kompakt durch die $2 n_{\mathcal{B}} \times 2 n_{\mathcal{B}}$ dimensionale (gerichtete) Konnektivitätsmatrix $\mathbf{F}$ darstellen. Dabei ist $\mathbf{F}_{d d^{\prime}}=1$, wenn die Kante $d^{\prime}$ der Kante $d$ folgt. Sonst verschwindet $\mathbf{F}_{d d^{\prime}}$.

\section{Dynamik auf Graphen}

Es soll nun zunächst die quantenmechanische und dann die dazu korrespondierende klassische Dynamik auf Graphen eingeführt werden. Die Wellenfunktion $\Psi$ ist ein Vektor $\Psi=\left(\Psi_{1}, \ldots, \Psi_{n_{\mathcal{B}}}\right)$, wobei die Funktion $\Psi_{b}(x)$ auf der Kante $b$ lebt. Auf den Kanten soll die freie Schrödingergleichung $(\hbar=2 m=1)$

$$
-\partial_{x}^{2} \Psi_{b}(x)=k^{2} \Psi_{b}(x)
$$

gelten. Die allgemeine Lösung erhält man als Überlagerung ebener Wellen

$$
\Psi_{b}(x)=a_{b} e^{\imath k x}+\hat{a}_{b} e^{-\imath k x} .
$$

Die $2 n_{\mathcal{B}}$ Koeffizienten $a_{b}$ und $\hat{a}_{b}$ werden durch die Randbedingungen, die die Wellenfunktionen an den Knoten erfüllen müssen, spezifiziert. Diese müssen so gewählt werden, dass der Schrödinger-Operator selbstadjungiert ist. Für jeden Knoten $v$ gibt es $d(v)$ ebene Wellen, die zu diesem Knoten laufen. Entsprechend gibt es auch genau $d(v)$ ebene Wellen, die von dem Knoten ausgehen. Da der Schrödinger-Operator selbstadjungiert ist, muss an jedem Knoten der Fluss erhalten sein - der Fluss der auslaufenden Wellen muss gleich dem einlaufenden Fluss sein. Damit lässt sich der Streuprozess an jedem Knoten $v$ durch unitäre Matrizen $\sigma_{v}$ der Dimension $\mathrm{d}(v) \times \mathrm{d}(v)$ beschreiben. Durch die Angabe der $n_{\mathcal{V}}$ Streumatrizen $\sigma_{v}$ der Knoten ist das Eigenwertproblem Gl. (5.16) eindeutig bestimmt. Die Eigenwerte 


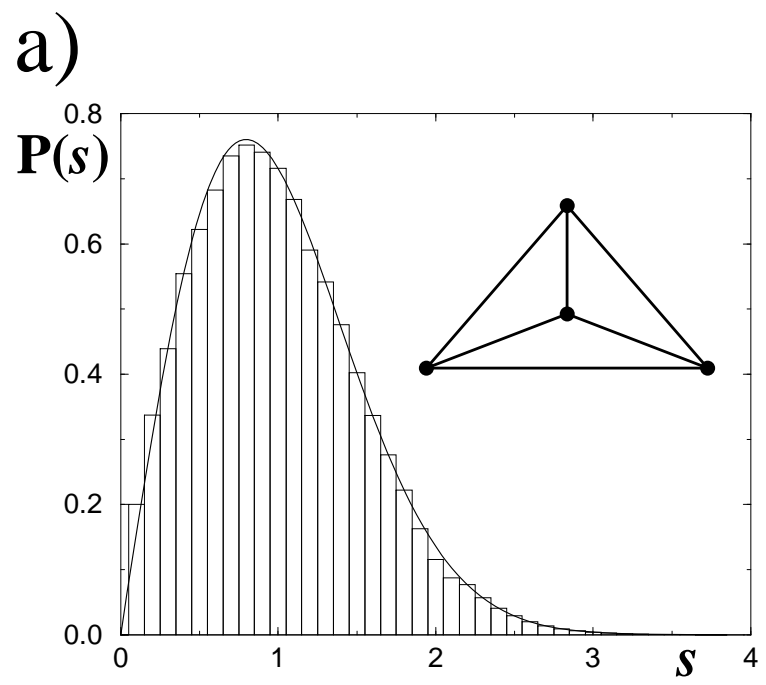

b)

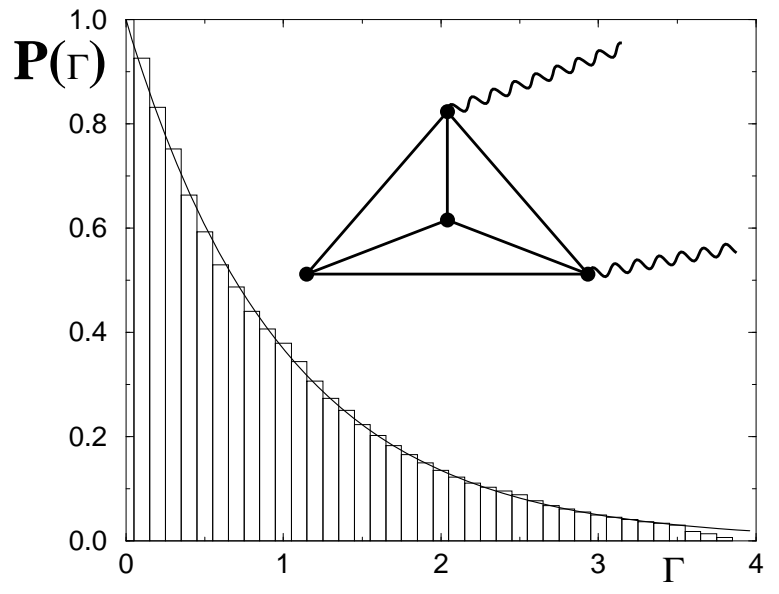

Abbildung 5.5: a) Abstandsverteilung benachbarter Energieniveaus für einen Graphen in Form eines Tetraeders. Um die Verteilung zu bestimmen, wurden $1.5 \cdot 10^{5}$ Eigenwerte numerisch berechnet. Die Verteilung folgt in guter Näherung der Theorie der Zufallsmatrizen für zeitinvariante Systeme. b) An zwei der vier Knoten des Tetraeders wurden Kanäle angebracht. Die Transmissionswahrscheinlichkeit $t$ vom Lead in den Tetraeder wurde so gewählt, dass der Graph nur schwach an die Kanäle koppelt $(t=0.05)$. So kann jedem Eigenwert aus a) eine Resonanzbreite $\Gamma$ zugeordnet werden. Die Verteilung der Resonanzbreiten $P(\Gamma)$ folgt gut der Zufallsmatrizentheorie für schwache Koppelungen, die für zwei Kanäle einen exponentiellen Abfall vorhersagt.

finden sich als Lösungen der Gleichung

$$
\operatorname{det}(\mathbf{I}-\mathbf{U}(k))=0
$$

Dabei ist $\mathbf{U}(k)$ die $2 n_{\mathcal{B}} \times 2 n_{\mathcal{B}}$ dimensionale unitäre Matrix

$$
\mathbf{U}_{d d^{\prime}}(k)=\mathbf{F}_{d d^{\prime}} e^{\imath k l_{d}} \sigma_{d d^{\prime}}
$$

Gleichung 5.18 in Verbindung mit Gl. (5.19) hat die folgende anschauliche Interpretation: Einem Eigenvektor $w$ zu einem Eigenwert $k^{2}$ entspricht eine Verteilung auf den Kanten. Diese Verteilung wird zunächst gestreut gemäß der Streumatrizen $\sigma$. Danach erhält jeder Eintrag eine Phase entsprechend der Länge der Kante, zu der er gehört. Werden die Kanten gemäß $\mathbf{F}_{d d^{\prime}}$ umsortiert, so muss - bis auf eine Normierung - wieder der ursprüngliche Vektor herauskommen, damit $w$ ein Eigenvektor ist. Die Eigenwerte findet man nun, in dem man die Nullstellen von Gl. (5.18) bestimmt. Durch die Transformation $k=\sqrt{E}$ wird das Spektrum entfaltet und der mittlere Niveauabstand $\Delta=\pi / L$ hängt nur von der Gesamtlänge des Graphen ab. Stehen die Kantenlängen zueinander in rationalen Verhältnissen, so ist das Spektrum periodisch. Interessanter ist der Fall, wenn die Längen inkommensurabel sind, was im Folgenden stets angenommen wird. Für vollständig verbundene Graphen konnten Kottos und Smilansky zeigen [94], dass die Abstandsverteilung benachbarter Niveaus in guter Näherung GOE verteilt sind (s. Abb. 5.5a). 
Quantengraphen können auch in ein Streuproblem umgewandelt werden [95]. Dazu werden, wie in Abb. 5.5b zu sehen ist, an einem Teil der Knoten eine oder mehrere Kanten (sogenannte Kanäle) angebracht, die nach unendlich verlaufen. Das Streuproblem wird durch die Streumatrix $S(k)$ beschrieben, deren Dimension durch die Anzahl der Kanäle festgelegt wird und sich als Lösung eines linearen Gleichungssystems ergibt. Die Streumatrix lässt sich eindeutig schreiben als

$$
S(k)=\frac{I-\imath A(k)}{I+\imath A(k)} .
$$

Die Pole der $S$-Matrix findet man als Nullstellen der Gleichung $\operatorname{det}(I+\imath A(k))=0$ in der komplexen $k$-Ebene. In Abb. 5.5b ist eine numerisch berechnete Verteilung von Resonanzbreiten für einen Tetraeder mit zwei Kanälen gezeigt. Die Verteilung folgt in guter Übereinstimmung der Zufallsmatrizentheorie für schwache Koppelungen.

Die zur Quantenmechanik korrespondierende klassische Dynamik wird folgendermaßen eingeführt. Auf den Kanten erfolgt die freie Zeitentwicklung ohne Potential. Entsprechend der Streumatrizen $\sigma$ gibt es klassische Wahrscheinlichkeiten $\left|\sigma_{d d^{\prime}}\right|^{2}$ für den Übergang von der Kante $d$ auf die Kante $d^{\prime}$. Bei der klassischen Dynamik handelt es sich also um einen Markov Prozess.

\subsubsection{Hierarchische Quantengraphen}

Aufbauend auf den Grundbegriffen der Quantengraphen soll nun ein Graphenmodell für die Dynamik im gemischten Phasenraum konstruiert werden. Die Konstruktion soll anhand dreier Kriterien erfolgen:

- Wie das klassische Kettenmodell, soll auch der Graph eine selbstähnliche Kettenstruktur besitzen, bestehend aus Regionen, die paarweise gekoppelt sind.

- Die einzelnen Regionen sollen die chaotische Dynamik zwischen den partiellen Transportbarrieren im Phasenraum wiederspiegeln.

- Die Mixing-Zeit jeder Region soll kleiner sein als die mittlere Verweilzeit in der Region.

Ein Graph, der diese Kriterien erfüllt, ist in Abb. 5.6b gezeigt. Er besteht aus gekoppelten Teilgraphen mit jeweils $v$ Knoten, die vollständig vernetzt sind, was die Mixing-Zeit minimiert. Weiterhin wurde oben gezeigt, dass solche vollvernetzten Graphen quantenmechanisch Signaturen chaotischer Dynamik aufweisen. Die Längen der Teilgraphen $L_{n}$ entsprechen den Volumina $\Omega_{n}$ und folgen daher dem Skalierungsverhalten $L_{n} \sim \omega^{n}$ wie im Kettenmodell. Die Hälfte der Kanten des $n$-ten Teilgraphen sind über gemeinsame Knoten mit Kanten der $n-1$-ten Stufen bzw. der $n+1$-Stufe verbunden. Die $v / 2$ Streumatrizen 
a)

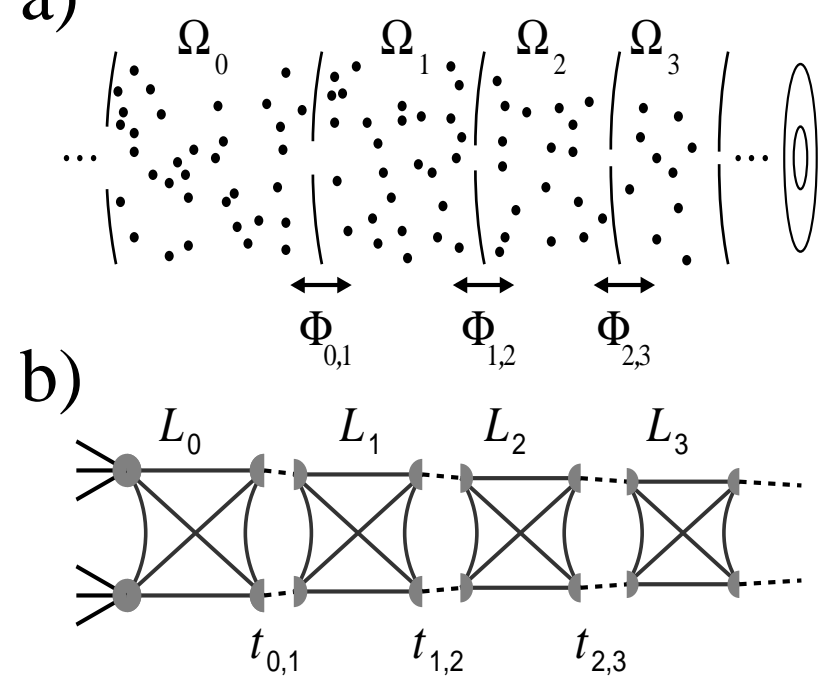

c)

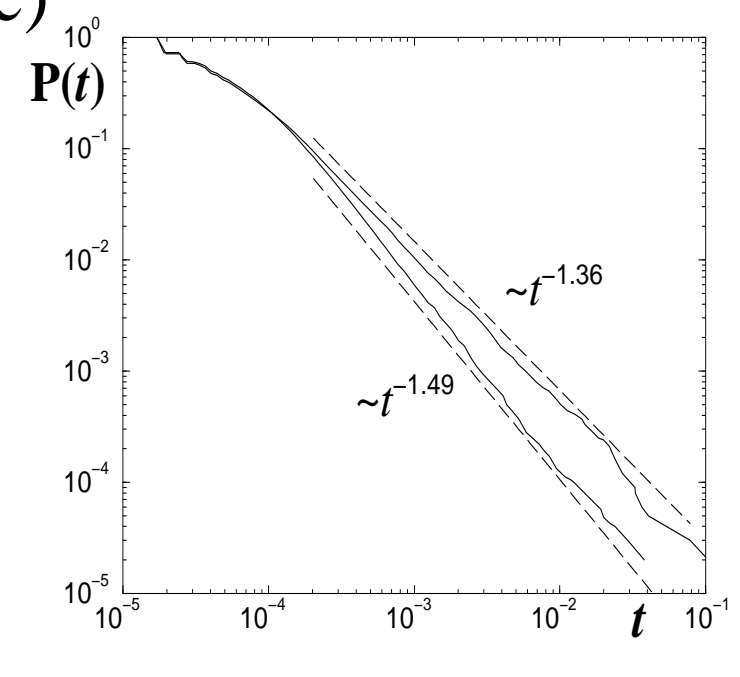

Abbildung 5.6: a) Skizze des gemischten Phasenraums mit Volumina $\Omega_{n}$, die über Flüsse $\Phi_{n, n+1}$ miteinander verbunden sind. b) Realisierung des Kettenmodells mit Hilfe von Quantengraphen mit $v=4$ Knoten pro Teilgraph. Die Gesamtlänge $L_{n}$ eines Teilgraphen entspricht dem Volumen $\Omega_{n}$, die Flüsse werden durch Transmissionswahrscheinlichkeiten $t_{n, n+1}$ realisiert. An der obersten Hierarchiestufe sind Kanäle angebracht, die den Graphen in ein Streuproblem verwandeln. c) Klassische Aufenthaltwahrscheinlichkeit im hierarchischen Quantengraphen $(v=4)$ für verschiedene Skalierungsparameter $\left(\omega_{1}=0.6, \varphi_{1}=0.15\right.$ und $\left.\omega_{2}=0.6, \varphi_{2}=0.21\right)$. Es wurden $10^{5}$ Teilchen im ersten Teilgraphen mit fester Geschwindigkeit gestartet und so lange iteriert, bis sie den Graphen über die Kanäle verlassen haben. Die gestrichelten Linien geben den zu erwartenden Zerfall $\gamma=1 /(1-\ln \omega / \ln \varphi)$ an.

der Verbindungsknoten müssen so gewählt werden, dass die klassischen Flüsse des Kettenmodells nachgeahmt werden. Die folgenden unitären Matrizen tragen dieser Bedingung Rechnung

$$
\sigma=\left(\begin{array}{cc}
r U & t U \\
-t U^{\dagger} & r U
\end{array}\right)
$$

Die Matrix $\sigma$ hat die Dimension $\operatorname{dim}(\sigma)=v(v-1) / 2$. Gilt $r^{2}+t^{2}=1$, so ist $\sigma$ für beliebige unitäre Matrizen $U$ selbst unitär. Soll keine Kante ausgezeichnet sein, ist es sinnvoll $U$, so zu wählen, dass alle Einträge den selben Absolutwert $2 / \sqrt{v(v-1)}$ haben und sich nur in den Phasen unterscheiden. Der Fluss $\Phi_{n, n+1}$ zwischen den Regionen $n$ und $n+1$ übersetzt sich in die Transmissionswahrscheinlichkeit $t_{n, n+1}$ der Matrizen $\sigma$. Im klassischen Kettenmodell gibt es neben den Skalierungsparametern $\omega, \varphi$ noch den Fluss $\Phi_{0}$, mit dem die Kette nach außen koppelt. Dies wird im Graphenmodell durch Kanäle an der ersten Hierarchiestufe realisiert, wobei die Koppelung an die Kanäle entsprechend des klassischen Flusses $\Phi_{0}$ gewählt wird. Im Gegensatz zum Kettenmodell besitzt die Realisierung mit Quantengraphen als zusätzlichen Parameter noch die Anzahl $v$ der Knoten in jeder Hierarchiestufe. 


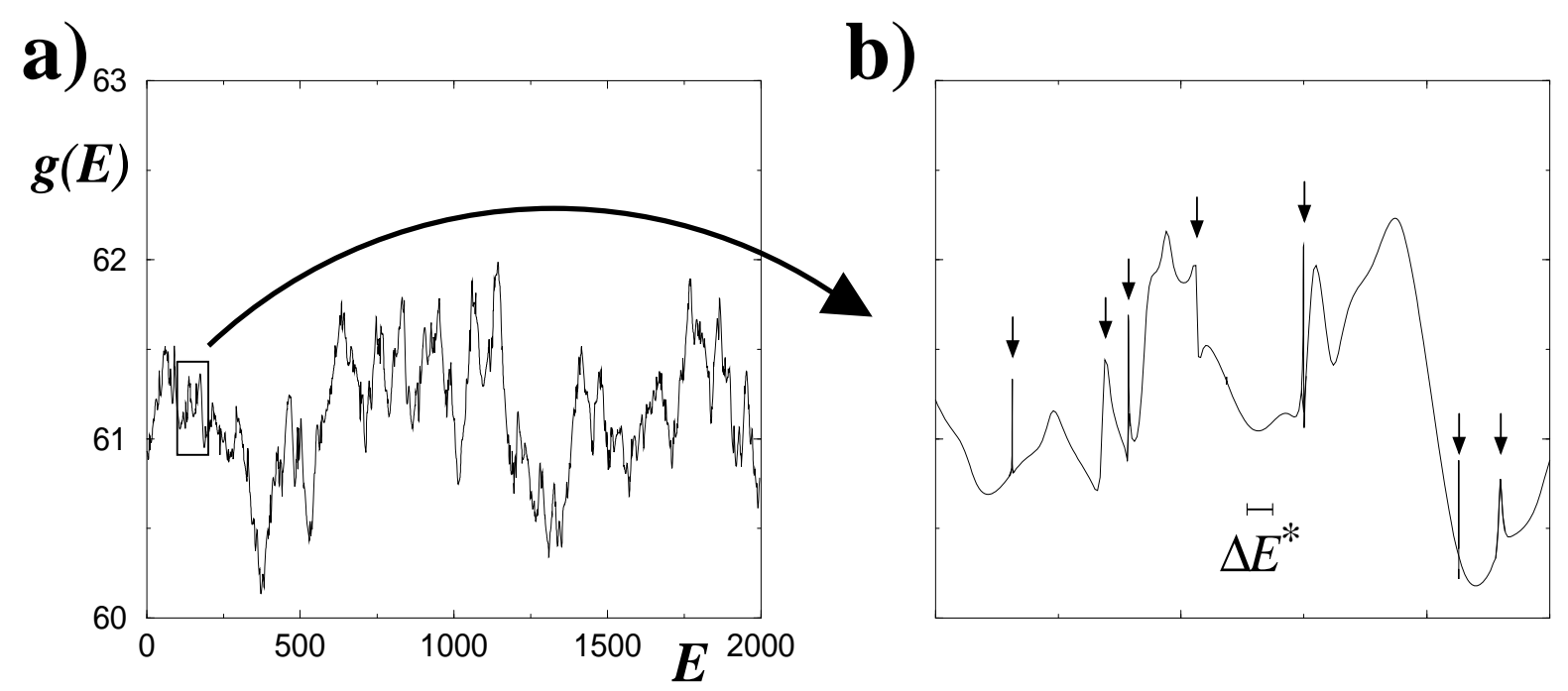

Abbildung 5.7: Leitwertkurve $g(E)$ für eine hierarchischen Quantengraphen mit $v=32(\omega=$ $0.6, \varphi=0.21$ ). Auf großen Energieskalen findet man FLF (links), auf kleinen Skalen (rechts) sind isolierte Resonanzen zu sehen.

Der Fluss zwischen benachbarten Regionen hängt gemäß

$$
\Phi_{n, n+1}=\frac{1}{2} t_{n, n+1}(v-1) \frac{v}{2}
$$

von $v$ ab. Diese Formel kommt folgendermaßen zu Stande. Zunächst läuft ein Teilchen in Region $n$ mit der Wahrscheinlichkeit $1 / 2$ auf einen Knoten der $(n+1)$-ten Region zu. An jedem Knoten kommen $v-1$ Kanten an. Insgesamt gibt es $v / 2$ Verbindungsknoten. Auf die klassische Dynamik hat $v$ keinen Einfluss. Für die nachfolgenden quantenmechanischen Untersuchungen wird $v$ jedoch eine wichtige Rolle spielen.

\subsubsection{Leitwerte für Quantengraphen}

Zunächst soll überprüft werden, ob die klassische Dynamik auf dem Graphen das algebraische Hängenbleiben im gemischten Phasenraum korrekt beschreibt. Dazu werden für verschiedene Skalierungsparameter Ensemble von Teilchen in der ersten Hierarchiestufe gestartet und solange evolviert, bis sie den Graphen über die Kanäle verlassen haben. Der so ermittelte Zerfall der Aufenthaltswahrscheinlichkeit im Graphen ist für zwei Beispiele in Abb. 5.6c dargestellt. Die beobachteten Zerfallsexponenten sind in guter Übereinstimmung mit den Vorhersagen des Kettenmodells Gl. (4.8).

Es soll nun das quantenmechanische Streuproblem untersucht werden. Wie oben schon erwähnt wurde, spielt die Anzahl der Knoten $v$ pro Hierarchiestufe eine wichtige Rolle. Bei festgehaltenen Skalierungsparameter $\omega$ und $\varphi$ bestimmt $v$ die Lage der Flussbarriere. Im 
eindimensionalen Graphenmodell folgt sie aus der Beziehung $\Phi_{n, n+1}=\hbar^{0}=1$. Mit Hilfe von Gl. (5.22) und dem Skalierungsverhalten $t_{n, n+1}=t_{0} \varphi^{n}$ ergibt sich

$$
n^{*}=\frac{\ln 4 /\left(t_{0} v(v-1)\right)}{\ln \varphi},
$$

wobei die Transmissionswahrscheinlichkeit von den Kanälen in den Graphen $t_{0}>4 /(v(v-$ 1)) erfüllen muss, da sonst schon die erste Region schwach an die Kanäle koppelt. Für alle untersuchten hierarchischen Graphen wurde $t_{0}=0.5$ gewählt. In Abb. 5.7 ist der Leitwert für einen Graphen mit $v=32(\omega=0.6, \varphi=0.21)$ und insgesamt 10 Hierarchiestufen als Funktion der Energie dargestellt. Für diese Parameter liegt die Flussbarriere zwischen den Regionen $n=3$ und $n=4$. Entsprechend der obigen Theorie findet man auf großen Energieskalen FLF und auf kleinen Skalen isolierte Resonanzen. Die Energieskala $\Delta E^{*}$, die die isolierten Resonanzen von den FLF trennt, erhält man gemäß Gl. (5.12) aus der Zeitskala $t^{*}$, der klassischen Zeitskala an der Flussbarriere. Das Skalierungsverhalten des betrachteten Graphen liefert $\Delta E^{*}=14.8 \Delta$. Dies ist in guter Übereinstimmung mit der numerisch berechneten Leitwertkurve (Abb. 5.7) und bestätigt die theoretischen Vorhersagen.

Das Graphenmodell für den gemischten Phasenraum erlaubt es sogar, den Einfluss der einzelnen Regionen auf den Leitwert getrennt zu untersuchen. Dazu beginnt man zunächst mit nur einer Hierarchiestufe und hängt dann nach und nach weitere Stufen an den Graphen an. Die Leitwertkurven, die man so für einen Graphen mit $v=32$ erhält, sind in Abb. 5.8 zusammengestellt. Die Regionen vor der Flussbarriere sorgen für FLF. Die erste Region liefert Fluktuationen auf großen Skalen. Jede weitere Region verursacht weitere Fluktuationen, die immer kleiner werden, je tiefer die Region in der Hierarchie liegt. Regionen nach der Flussbarriere liefern isolierte Resonanzen im Leitwert. Die Anzahl der isolierten Resonanzen, die jeweils neu hinzukommen, sowie deren Breite nimmt schnell mit der Tiefe der Region in der Kette ab. Da die Regionen hinter der Flussbarriere zu isolierten Resonanzen führen, kann man eine Verbindung zwischen der Lage der Resonanz und den Eigenwerten des geschlossenen Systems herstellen. Setzt man die Transmissionswahrscheinlichkeiten $t_{n-1, n}$ und $t_{n, n+1}$ auf Null, so können mit Hilfe von Gl. (5.18) die Eigenwerte der $n$-ten Region bestimmt werden. Nur so ist es effizient möglich, die sehr kleinen schmalen $\left(\Gamma<10^{-6} \Delta\right)$ Resonanzen aufzuspüren. Für Regionen vor der Flussbarriere ist eine solche Zuordnung nicht möglich.

Um die statistischen Eigenschaften der isolierten Resonanzen besser untersuchen zu können, ist es zweckmäßig einen Graphen zu betrachten, bei dem die Flussbarriere möglichst nahe am Anfang der Kette liegt. In Abb.5.9b ist der Leitwert für einen Graphen mit $v=4$ Knoten pro Hierarchiestufe gezeigt. Die Flussbarriere liegt hier hinter der ersten Region und man findet daher viele isolierte Resonanzen auf einer langsam variierenden Kurve. Nach den obigen Ausführungen ist klar, dass dieser Graph keine FLF zeigen kann. Die isolierten Resonanzen werden durch ihre Resonanzbreite $\Gamma$ beschrieben. In Abb. 5.9a ist die Verteilung $P(\Gamma)$ der Resonanzbreiten für den Graphen dargestellt. Die Verteilung erstreckt sich über viele Größenordnungen und für kleine $\Gamma$ nähert sie sich $1 / \Gamma$ an, was man 


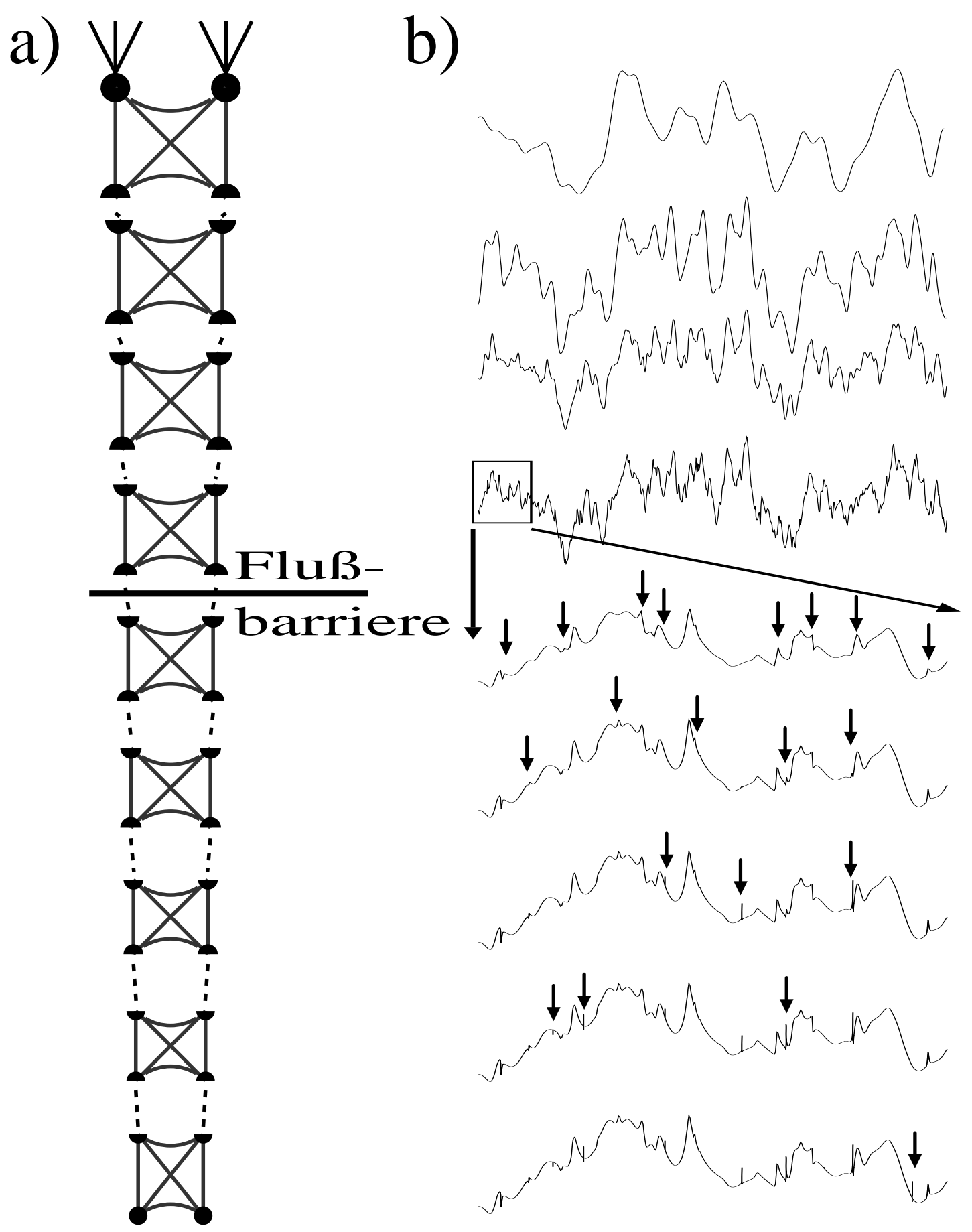

Abbildung 5.8: a) Gezeigt ist eine Skizze des hierarchischen Quantengraphen zusammen mit der Lage der Flussbarriere für einen Graphen mit $v=32$ (zwischen $n=3$ und $n=4$ ). Um das Bild übersichtlich zu halten, wird anstatt eines Graphen mit $v=32$ Konten ein Graph mit 4 Knoten pro Hierarchiestufe gezeigt. b) Leitwertkurven für einen hierarchischen Quantengraphen mit $v=32$. Die Anzahl der Hierarchiestufen nimmt von oben nach unten zu. Die Regionen bis zur Flussbarriere verursachen Fluktuationen auf der gesamten Breite der Kurve, wobei diese immer feiner werden. Regionen hinter der Flussbarriere führen zu isolierten Resonanzen. Die Pfeile markieren die Resonanzen, die jeweils neu hinzukommen, wenn eine weitere Region angehängt wird. 

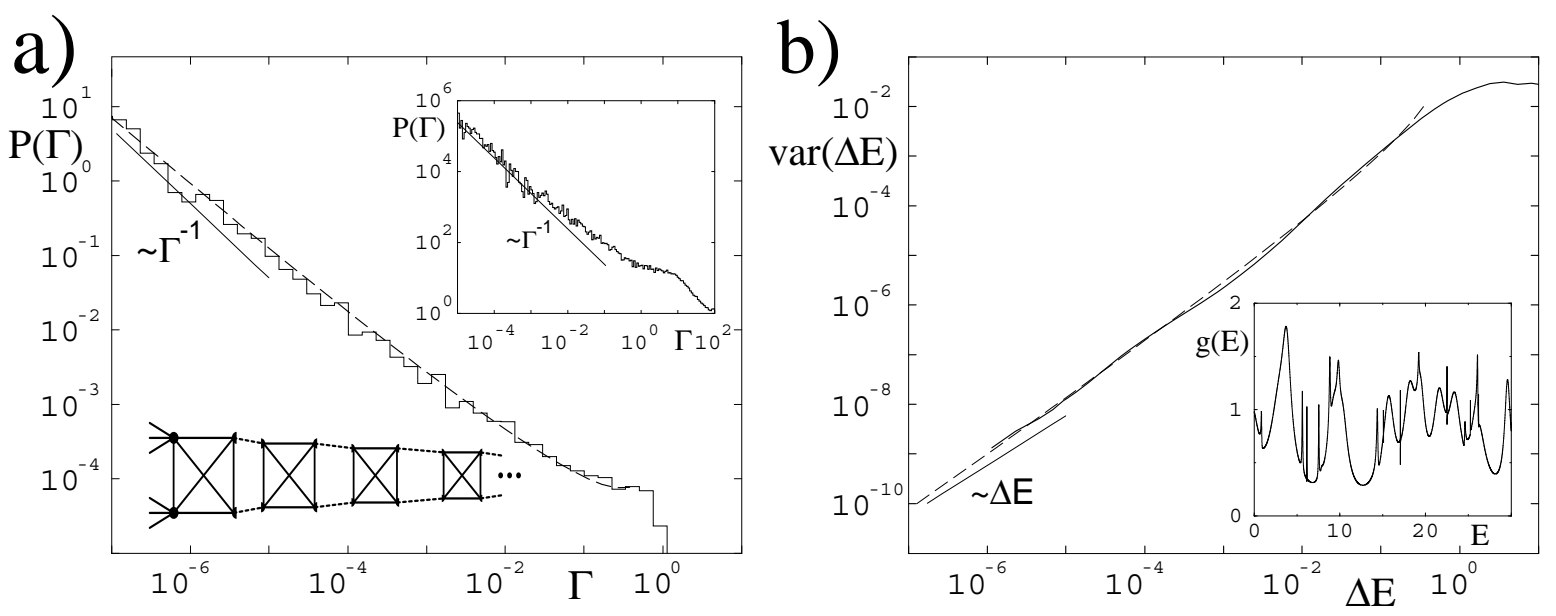

Abbildung 5.9: a) Verteilung der Resonanzbreiten $p(\Gamma)$ für eine hierarchischen Quantengraphen mit $v=4$ Knoten pro Hierarchiestufe und $\Omega=0.6$ und $\varphi=0.21$. Es gibt einen breiten Übergangsbereich zum asymptotischen Verhalten $p(\Gamma) \sim 1 / \Gamma$ für kleine $\Gamma$. Die gestrichelte Kurve zeigt die Vorhersage aus Gl. (5.15) für die verwendeten Parameter. Das Inset zeigt die entsprechende Verteilung für den gekickten Rotator. b) Verteilung der Leitwertinkremente var $(\Delta E)$ für den Graphen aus a). Die gestrichelte Kurve zeigt die Erwartung gemäß der Verteilung $p(\Gamma)$. Für kleine $\Delta E$ wächst die Varianz linear an. Das Inset zeigt die Leitwertkurve für den Graphen.

auch für gekickte Systeme mit gemischtem Phasenraum findet (s. Inset Abb. 5.9a). Jedoch gibt es einen breiten Übergangsbereich, der von den Skalierungsparametern abhängt. Die Kurve wird erstaunlich gut durch Gl. (5.15) beschrieben. Jede Resonanz $\Gamma$ führt zu einem isolierten Merkmal der Breite $\sim \Gamma$ im Leitwert. Unter der Annahme, dass die Höhe und Breite dieser einzelnen Merkmale unkorreliert sind, konnte in Ref. [35] gezeigt werden, dass ein Potenzgesetz $P(\Gamma) \sim \Gamma^{\delta}$ zu einem Potenzgesetz $\operatorname{var}(\Delta E) \sim \Delta E^{\delta+2}$ in der Verteilung der Inkremente des Leitwerts führt. Das asymptotische Potenzgesetz $1 / \Gamma$ führt demnach zu einem linearen Anstieg $\operatorname{var}(\Delta E) \sim \Delta E$ für kleine $\Delta E$, was man auch für den Quantengraph findet, wie man in Abb. 5.9b sieht.

\subsection{Zusammenfassung und Ausblick}

In diesem Kapitel wurde gezeigt, dass die zwei beobachteten und sich scheinbar widersprechenden Typen von Leitwertfluktuationen - FLF [34,75-79] und isolierte Resonanzen [35] ihre gemeinsame Ursache in der klassischen Struktur des gemischten Phasenraums haben. Im Allgemeinen treten beide Typen gleichzeitig - jedoch auf unterschiedlichen Energieskalen - auf. Die Regionen vor der Flussbarriere führen zu FLF und Regionen dahinter verursachen die isolierten Resonanzen. Für die isolierten Resonanzen konnte eine Verbindung zu den hierarchischen Eigenzuständen des geschlossenen Systems hergestellt werden. 
Die Energieskala, die beide Typen trennt, ist durch die klassische Rate an der Flussbarriere gegeben. Durch Variation des effektiven Planckschen Wirkungsquantum verschiebt sich die Lage der Flussbarriere und so kann das Gewicht zwischen FLF und isolierten Resonanzen verändert werden. Im semiklassischen Limes $\hbar_{\text {eff }} \rightarrow 0$ überwiegen die FLF.

Die Ergebisse dieses Kapitels beruhen - abgesehen von der numerischen Verifikation der der Zeitskala $t^{*}$ - auf Untersuchungen eines Quantengraphenmodells des gemischten Phasenraums. Eine Verifikation an Hamiltonschen Systemen mit gemischten Phasenraum ist daher wünschenswert. Zunächst sollte am Cosinus-Billard der Zusammenhang zwischen der Lage der isolierten Resonanzen und den Eigenenergien der hierarchischen Eigenzustände des geschlossenen Billards überprüft werden. Insbesondere müsste sich für kleineres $\hbar_{\mathrm{eff}}$ (Vergrößerung des Billards) die Anzahl der isolierten Resonanzen verringern und die fraktalen Eigenschaften sollten sichtbar werden. Eine weitere Möglichkeit, diesen Übergang detailierten zu studieren, besteht in der Untersuchung von Streueigenschaften gekickter Systeme. Borgonovi und Guarneri haben gezeigt, wie solche Systeme in ein Streuproblem umformuliert werden können und anhand des gekickten Rotators den vollständig chaotischen Grenzfall untersucht [96-98].

Schliesslich wäre eine experimentelle Überprüfung der hier vorgestellten Ergebnisse wünschenswert. In typischen Experimenten an Halbleiter-Heterostrukturen wird der Leitwert als Funktion eines externen Magnetfeldes gemessen. Die Feldstärke $\Delta B^{*}$, die die fraktalen Fluktuationen von den isolierten Resonanzen trennt, ergibt sich aus der klassischen Zeitskala $t^{*}$ an der Flussbarriere: Eine Trajektorie, die bis zur Zeit $t^{*}$ im Billard verweilt, umschließt typischerweise eine Fläche $A^{*}$. Diese Fläche liefert die Feldstärke $\Delta B^{*}=h /\left(e A^{*}\right)$. Ist die Phasenkohärenzzeit $\tau_{\varphi}>t^{*}$, so sollte man auf Skalen unterhalb $\Delta B^{*}$ isolierte Resonanzen finden. 


\section{Kapitel 6}

\section{Kreuzförmiger Transport}

Nachdem in den vorherigen Kapiteln die semiklassische Analyse der Dynamik gemischter Systeme im Vordergrund stand, soll nun der Schwenk zu rein quantenmechanischen Transporteigenschaften vollzogen werden. Seit den grundlegenden Arbeiten von Bloch und Landau [99, 100] wurde der Zusammenhang zwischen spektralen und Transporteigenschaften von Elektronen in magnetischen Feldern intensiv untersucht. Während der idealisierte Fall eines freien Elektrons in einem statischen Magnetfeld zu unendlich entarteten LandauNiveaus führt, sorgt ein periodisches Potential ohne Feld für Bandstrukturen im Spektrum. Obwohl die Kombination von periodischem Potential und Magnetfeld ein scheinbar einfaches System liefert, ist die Dynamik überraschend kompliziert und zeigt viele interessante Effekte.

Eine einfache Realisierung eines zweidimensionalen periodischen Potentials ist durch

$$
V(\boldsymbol{r})=V_{x} \cos \left(\frac{2 \pi x}{a}\right)+V_{y} \cos \left(\frac{2 \pi y}{b}\right)
$$

gegeben, wobei $a, b$ die Gitterperioden in der $x y$-Ebene sind. Eine Einbandnäherung führt nach einer Separation der Variablen zum eindimensionalen Harper-Modell [101]

$$
\mathcal{H}_{\mathrm{HM}}=2 \cos (x)+\lambda \cos (p),
$$

wobei $\lambda=2 V_{y} / V_{x}$ gesetzt wurde. Dieses Modell wurde intensiv untersucht, und viele interessante spektrale Eigenschaften wurden gefunden [102-106]: Für $\lambda<2$ sind alle Zustände ausgedehnt, für $\lambda>2$ hingegen lokalisiert. Am kritischen Punkt $\lambda=2\left(V_{x}=V_{y}\right)$ ist das Spektrum multifraktal, was zu anomaler Diffusion führt. Ein Nachteil der Einbandnäherung ist, dass die Dynamik im klassischen Limes integrabel ist, die klassische Dynamik der Elektronen im Magnetfeld jedoch Chaos zeigt. Um der chaotischen Dynamik der Elektronen Rechnung zu tragen und dennoch zu numerisch handlichen Modellen zu gelangen, 
wurde das gekickte Harper-Modell (KHM) vorgeschlagen

$$
\mathcal{H}_{\mathrm{KHM}}=L \cos (p)+K \cos (x) \sum_{n \in \mathbb{Z}} \delta(t-n)
$$

Im Limes kleiner Kickstärken erhält man aus den Bewegungsgleichungen des KHM wieder die Harper-Gleichung. Die Dynamik des KHM ist sehr vielfältig. Sie reicht von Lokalisierung über Diffusion und anomale Diffusion bis zu ballistischem Transport [107-113]. Zwar ist das KHM mit Hilfe numerischer Methoden einfach zugänglich, jedoch ist die experimentelle Relevanz und Realisierbarkeit unklar. Iomin und Fishman haben jüngst gezeigt, dass Elektronen im periodischen Potential mit magnetischem Feld und einem zusätzlichen zeitlich alternierenden elektrischen Feld zu einer Vielzahl gekickter Modelle führen, die dem KHM ähnlich sind [114-116]. Sie beobachteten in der Dynamik einen Metall-Isolator-Übergang, der durch eine Variation der Amplitude des elektrischen Feldes hervorgerufen wird. Im Folgenden soll nun die Dynamik des Systems genauer untersucht und mit spektralen Eigenschaften in Verbindung gebracht werden [40].

\subsection{Das doppelt gekickte Harper-Modell}

Ausgangspunkt ist die Hamilton-Funktion

$$
\mathcal{H}=\frac{1}{2 m^{*}}[\boldsymbol{p}-e \boldsymbol{A}(\boldsymbol{r}, t)]^{2}+V(\boldsymbol{r})
$$

Dabei ist $\boldsymbol{p}=\left(p_{x}, p_{y}\right)$ der Impuls des Elektrons mit der effektiven Masse $m^{*}$ und der Ladung $e$. Die Bewegung des Elektrons erfolgt in der $x y$-Ebene senkrecht zum Magnetfeld der Stärke $B$. Zusätzlich zum konstanten Magnetfeld wirkt noch ein zeitlich mit Frequenz $\nu$ alternierendes elektrischen Feld $\boldsymbol{E}=\left(-E_{x} \sin \nu t, E_{y} \sin \nu t\right)$ auf das Elektron. In der Landau-Eichung hat das Vektorpotential die Form

$$
\boldsymbol{A}(\boldsymbol{r}, t)=\left(\frac{E_{x}}{\nu} \sin \nu t, x B-\frac{E_{y}}{\nu} \sin \nu t\right)
$$

Eine wichtige Größe ist die Zyklotronfrequenz $\omega^{*}=e B /\left(m^{*} c\right)$. Unter Verwendung der Variablentransformation $y \rightarrow y+e Y_{y} / m^{*} \nu^{2} \sin \nu t$ und $p \rightarrow p+e \omega^{*} / \nu^{2} E_{y} \sin \nu t$ können die Bewegungsgleichungen Gl. (6.4) unter der Bedingung $\omega^{*} E_{y}=\nu E_{x}$ durch die effektive Hamilton-Funktion

$$
\begin{aligned}
\mathcal{H}_{\mathrm{eff}} & =\frac{1}{2 m^{*}}\left[p_{x}^{2}+\left(p_{x}-\omega^{*} m^{*} x\right)^{2}\right] \\
& +V_{x} \cos \frac{2 \pi x}{a}+V_{y} \cos \left[\frac{2 \pi x}{b}\left(y+e E_{y} / m^{*} \nu^{2} \sin \nu t\right)\right] \\
& =\mathcal{H}_{0}(\mathbf{p}, x)+V(\mathbf{r}, t)
\end{aligned}
$$


beschrieben werden. Dabei beschreibt $\mathcal{H}_{0}(\mathbf{p}, x)$ die Zyklotronbewegung und die Störung $V(\mathbf{r}, t)$ hebt die unendliche Entartung auf und führt zu Minibändern. Für starke elektrische Felder und $\nu=1$ reduziert sich die Hamilton-Funktion Gl. (6.6) zu dem gekickten System $[114,115]$

$$
\tilde{\mathcal{H}}=L \cos p+K \sum_{n=-\infty}^{\infty}\left[\cos \left(q-\kappa_{0}\right) \delta(t-2 n+1)+\cos \left(q+\kappa_{0}\right) \delta(t-2 n)\right],
$$

wobei die kanonischen Operatoren $p, q$ zu $x$ und $y$ gehören und die Vertauschungsrelation $[p, q]=-\imath 2 \pi \hbar_{\mathrm{eff}}$ erfüllen. Das inverse effektive Plancksche Wirkungsquantum

$$
\hbar_{\mathrm{eff}}^{-1}=(a b e B) / \hbar
$$

gibt die Anzahl der Flussquanten pro Einheitszelle an. Der Phasenunterschied

$$
\kappa_{0}=\left(2 \pi e E_{y} /\left(b m^{*}\right)-\pi / 4\right) \bmod 2 \pi
$$

aufeinander folgender Kicks hängt von der elektrischen Feldstärke ab. Die Größen $L, K$ sind die skalierten Amplituden des Potentials aus Gl.(6.1).

\subsection{Numerische Behandlung}

Der Zeitentwicklungsoperator des doppelt gekickten Harper-Modells Gl. (6.7) für eine Periode ist durch

$$
\begin{aligned}
\mathcal{U}= & \exp \left(-\frac{\imath L}{\hbar_{\mathrm{eff}}} \cos p\right) \exp \left(-\frac{\imath K}{\hbar_{\mathrm{eff}}} \cos \left(q-\kappa_{0}\right)\right) \\
& \times \exp \left(-\frac{\imath L}{\hbar_{\mathrm{eff}}} \cos p\right) \exp \left(-\frac{\imath K}{\hbar_{\mathrm{eff}}} \cos \left(q+\kappa_{0}\right)\right) .
\end{aligned}
$$

gegeben. Die Zeitentwicklung eines Wellenpaketes erfolgt zweckmäßigerweise mit Hilfe von Fourier-Transformationen. Der einzige Unterschied zu "normalen" gekickten Systemen ist, dass hier zweimal je Periode zwischen Orts- und Impulsdarstellung gewechselt werden muss.

Es soll kurz auf die Berechnung des Spektrums eingegangen werden. Da das Potential periodisch ist, sind die Impulseigenzustände diskret. Diese werden mit $|m\rangle$ bezeichnet und erfüllen $p|m\rangle=\hbar\left(m+\theta_{q} / 2 \pi\right)|m\rangle$ mit der Bloch-Phase $\theta_{q}$. Ist $\hbar / 2 \pi=M / N$ rational, so ist das System translationsinvariant nach $N$ Schritten und der unendlichdimensionale Zeitentwicklungsoperator kann auf eine $N \times N$ Matrix reduziert werden [57]. Dafür muss man eine weitere Phase $\theta_{p}$ in Kauf nehmen, die die Anschlussbedingung nach $N$ Schritten angibt. Ist das effektive Wirkungsquantum irrational, so nähert man $\hbar / 2 \pi$ durch rationale Approximanden, die man aus der Kettenbruchentwicklung erhält. Das Spektrum von $\mathcal{U}$ erhält 

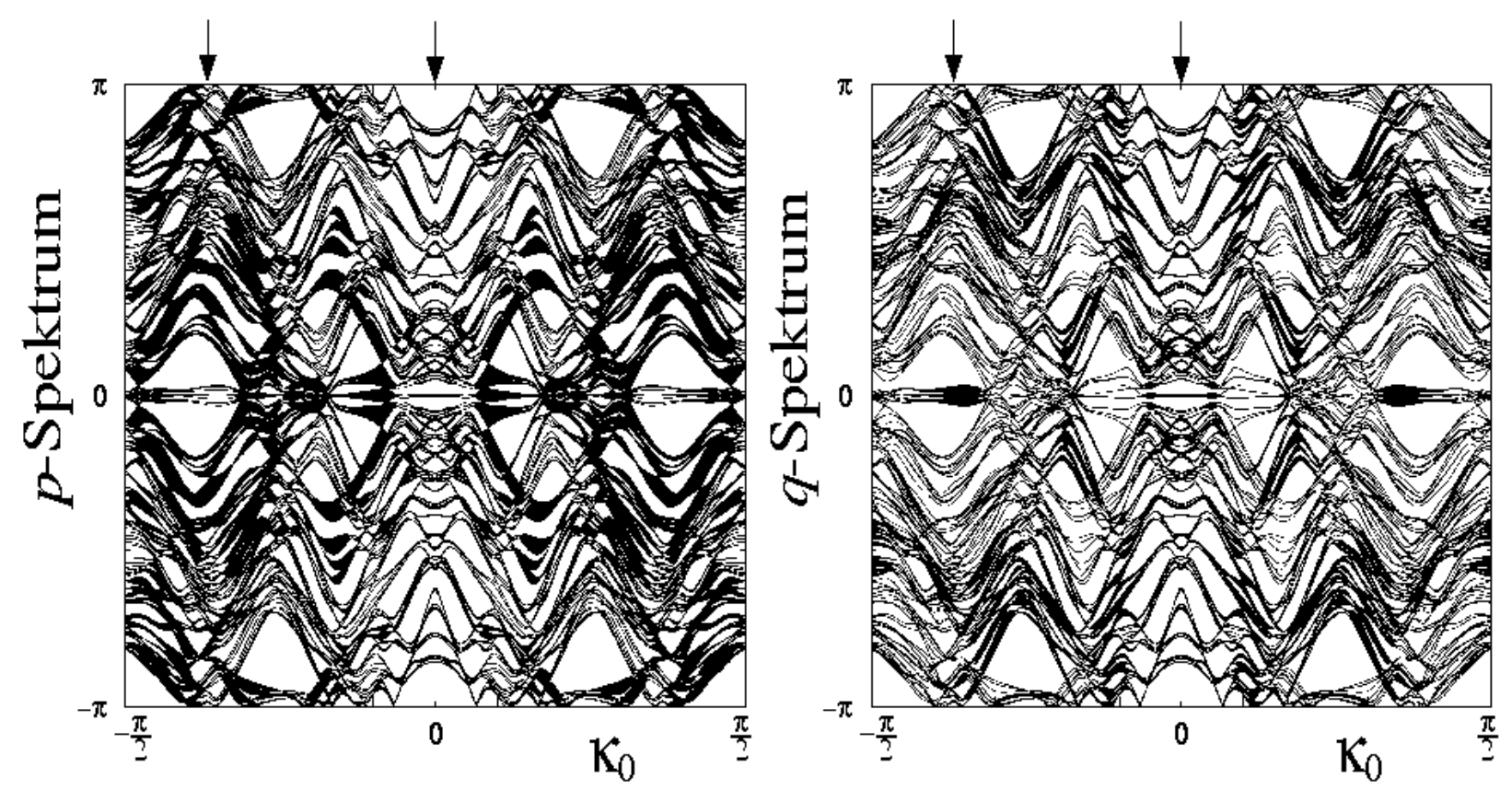

Abbildung 6.1: Das $p$-Spektrum (links) und $q$-Spektrum (rechts) für den Zeitentwicklungsoperator Gl. (6.10) für $K=L=5$ ist als Funktion von $\kappa_{0}$ dargestellt. Als Approximand für das effektive Wirkungsquantum hat sich $\hbar_{\text {eff }}=2 \pi 34 / 259$ als ausreichend erwiesen. Die Pfeile kennzeichnen die speziellen Werte $\kappa_{0}=0$ und $\kappa_{0}=\pi \sigma_{q}$, die in Ref. [116] verwendet wurden. Für $\kappa_{0}=0$ ist das Spektrum multifraktal, während es für $\kappa_{0} \neq 0$ aus Bändern und Niveaus besteht.

man durch Diagonalisierung der $N \times N$ Matrizen für alle Kombinationen der Bloch-Phasen $\theta_{p}, \theta_{q} \in[0,2 \pi]$. Hält man eine der beiden Phasen fest und variiert die andere, so erhält man einen Teil (einen Schnitt) des Spektrums. Das Teilspektrum zur festgehaltenen Phase $\theta_{p}$ wird mit $p$-Spektrum bezeichnet, das Spektrum zu $\theta_{q}=$ const heißt $q$-Spektrum. Eigenenergien zu Eigenfunktionen, die in $p$-Richtung lokalisiert sind, werden nur sehr schwach durch eine Phasenänderung $\theta_{p}$ beeinflusst, wenn das System groß genug gewählt wurde, so dass die Lokalisierungslänge kleiner als die Systemgröße $N$ ist. Natürlich hat jedes Band eine endliche Breite. Für lokalisierte Zustände wird sie jedoch exponentiell kleiner, wenn man zu größeren Approximanden geht. Im Folgenden werden diese sehr schmalen Bänder daher als Niveaus bezeichnet. Hingegen ändern sich die Eigenenergien zu ausgedehnten Zuständen unter Variation der Phase. Entsprechendes gilt bei Veränderung von $\theta_{q}$. Die beiden Teilspektren geben daher Aufschluss über die Gestalt der Eigenfunktionen und damit über Transporteigenschaften in $p$ - und $q$-Richtung. Um den Transport im Phasenraum in $p$ - und $q$-Richtung über mehrere Einheitszellen zu beobachten, geht man folgendermaßen vor. Angenommen man möchte den Transport in $q$-Richtung über $r$ Einheitszellen studieren. Dann gibt es für die Bloch-Phase nach einer Einheitszelle die $r$ Möglichkeiten $\theta_{q}(j)=2 \pi j / r$ mit $j=0, \ldots, r-1$. Zu jeder Phase $\theta_{q}$ startet man ein am Platz $n_{0} \delta$ lokalisiertes Wellenpaket im Impulsraum mit Impuls $\hbar\left(n_{0}+j / r\right)$. Zur Zeit $t$ erhält man so 
die $r$ Vektoren $\left|m, t ; \theta_{q}(j)\right\rangle$. In der Ortsdarstellung sind diese Funktionen periodisch nach einer Einheitszelle mit der Phase $\theta_{q}(j)$. Bildet man nun die Überlagerung

$$
\left|m, t>=\frac{1}{\sqrt{r}} \sum_{j=0}^{r-1} e^{-\imath \theta_{q}(j) k}\right| m, t ; \theta_{q}(j)>,
$$

so ist für alle $k \in \mathbb{Z}$ das Startwellenpaket in der Einheitszelle $(k \bmod r)$ im Ortsraum lokalisiert. Im Impulsraum hat das Wellenpaket die Breite $\hbar_{\mathrm{eff}}$.

\subsection{Resultate}

Es soll nun der in Ref. [116] gefundene Metall-Isolator-Übergang genauer untersucht werden. Dabei werden die zwei Fälle $K=L$ und $K \neq L$ betrachtet. Für beide Fälle wird $\hbar_{\mathrm{eff}}=2 \pi /\left(7+\sigma_{q}\right)$ gesetzt.

Fall $K=L$

In Ref. [116] wurde die Dynamik von Wellenpaketen für $K=L=5$ und $\hbar_{\text {eff }}=2 \pi /\left(7+\sigma_{q}\right)$, wobei $\sigma_{q}=(\sqrt{5}-1) / 2$ der goldene Schnitt ist, untersucht. Dabei wurde ein Übergang von diffusiver zu ballistischer Ausbreitung von ursprünglich im Impulsraum $\delta$-lokalisierten Wellenpaketen gefunden, wenn $\kappa_{0}$ von 0 nach $\pi \sigma_{q}$ verändert wird. In Abb.6.2 ist das $p$ und $q$-Spektrum zu den obigen Parametern für eine Periode in $\kappa_{0}$ gezeigt. Zunächst fällt auf, dass die beiden Spektren zueinander dual sind. Dies bedeutet, dass Niveaus in einem Spektrum zu Bändern im anderen Spektrum werden und umgekehrt.

Wie man aus Abb. 6.2 entnimmt, wächst für $\kappa_{0}=0$ die Varianz des Wellenpakets nahezu linear mit der Zeit in $p$ - und $q$-Richtung an. Dies ist eine direkte Konsequenz der multifraktalen Eigenschaften von Spektrum und Eigenfunktionen, denn für $\kappa_{0}=0$ reduziert sich Gl. (6.10) auf das gekickte Harper-Modell, für das dieses Verhalten intensiv studiert wurde [107-110,117].

Um $\kappa_{0}=\pi \sigma_{q}$ erscheinen Bänder im $p$-Spektrum, was zu ballistischem Transport in $p$ Richtung führt. Neben den Bändern gibt es allerdings auch noch Niveaus im $p$-Spektrum bei $\kappa_{0}=\pi \sigma_{q}$. Daher gibt es auch Bänder im $q$-Spektrum, die dafür verantwortlich sind, dass sich ein Wellenpaket ballistisch in $q$-Richtung ausbreitet. Dies wird durch Abb. 6.2b bestätigt. Auf Grund der Dualität sind alle Eigenfunktionen in einer Richtung ausgedehnt und lokalisiert in der anderen. Ein typisches Startwellenpaket regt das gesamte Spektrum gleichermaßen an und vereint damit beide Typen von Eigenfunktionen. Das Wellenpaket breitet sich daher ballistisch in $p$ - und $q$-Richtung aus, und der Transport in HusimiDarstellung gleicht einem Kreuz. Die Breiten der beiden Zweige hängen von den Lokalisierungslängen ab. Die Amplitude spiegelt das Verhältnis von Bändern zu Niveaus im 

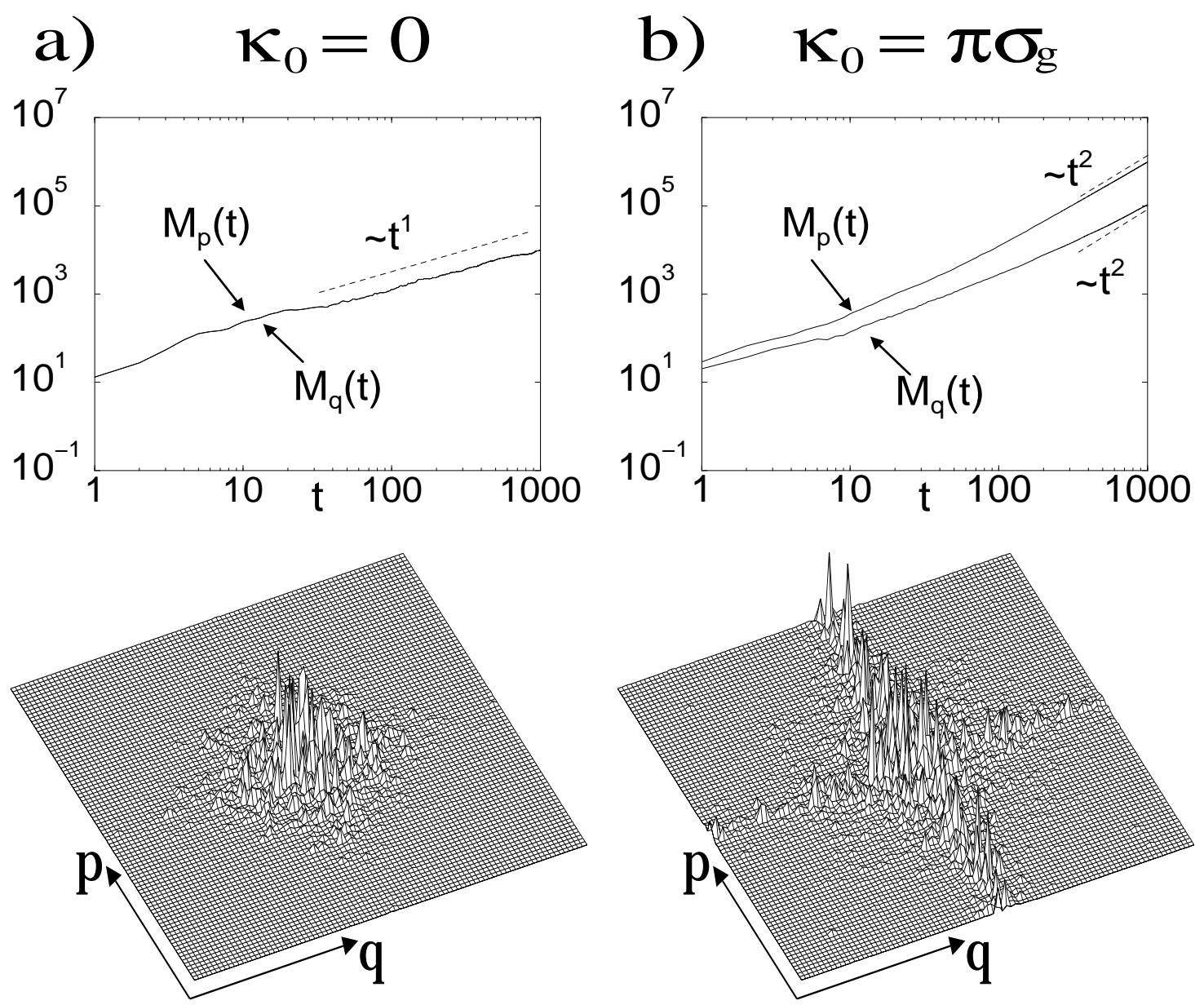

Abbildung 6.2: Die Varianzen $M_{p}(t)$ und $M_{p}(t)$ und die zugehörige Wellenfunktion nach 100 Kicks in Husimi-Darstellung zu den Parametern $K=L=5$ sind gezeigt. Für die HusimiDarstellung wurden 89 Einheitzellen in $p$ - und $q$-Richtung verwendet. a) Für $\kappa_{0}=0$ wachsen die Varianzen in $p$ - und $q$-Richtung nahezu diffusiv an. Das Wellenpaket breitet sich dabei isotrop aus. b) Für $\kappa_{0}=\pi \sigma_{q}$ findet man in beide Richtungen ballistische Ausbreitung des Wellenpakets. In Husimi-Darstellung hat das Wellenpaket die Form eines Kreuzes.

Spektrum wieder, wobei man die unterschiedlichen Lokalisierungslängen noch berücksichtigen muss.

Fall $K \neq L$

Für die Parameter $K=2$ und $L=4$ wurde in Ref. [116] ein Metall-Isolator-Übergang beobachtet, wenn $\kappa_{0}$ variiert wird. In Abb. 6.3 sind das $p$ - und $q$-Spektrum als Funktion von $\kappa_{0}$ wiedergegeben. Für $\kappa_{0}=0$ (KHM Fall) zeigt das $q$-Spektrum breite Bänder und entsprechend das $p$-Spektrum Niveaus. Man erwartet demnach ballistischen Trans- 

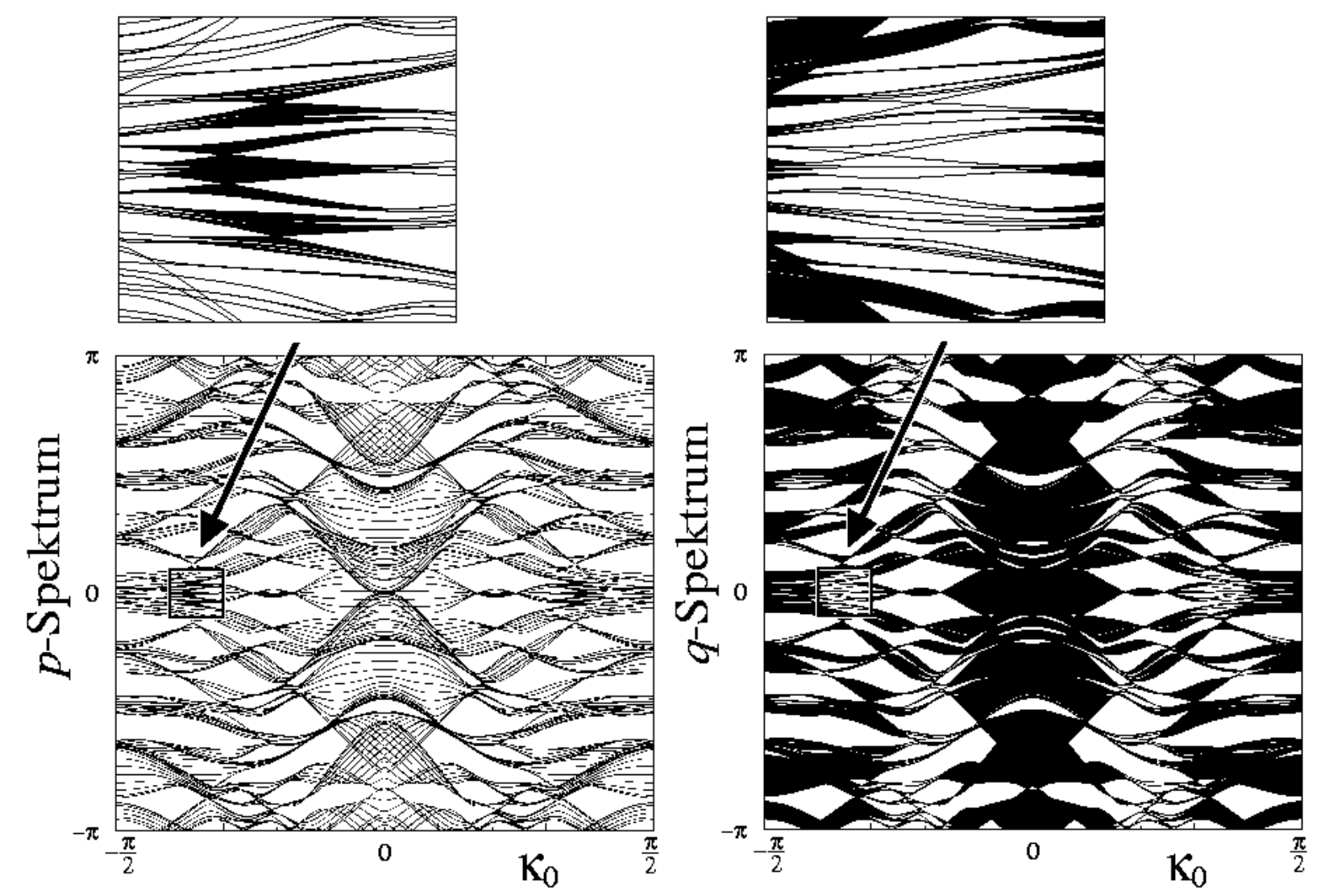

Abbildung 6.3: Das $p$-Spektrum (links) und $q$-Spektrum (rechts) für den Zeitentwicklungsoperator Gl. (6.10) für $K=2$ und $L=4$ und $\hbar_{\text {eff }}=2 \pi 34 / 259$ ist als Funktion von $\kappa_{0}$ dargestellt. Das $p$-Spektrum wird von Niveaus dominiert. Nur in einem kleinen Bereich um $\kappa_{0}= \pm \pi \sigma_{q}$ sind Bänder zu sehen. Die Vergrößerung zeigt einen Teil des Spektrums zum Approximanden $\hbar_{\mathrm{eff}}=2 \pi 34 / 259$. Deutlich ist der Übergang von Niveaus zu Bändern erkennbar. Entsprechendes gilt ebenfalls für das $q$-Spektrum.

port in $q$-Richtung und Lokalisierung in $p$-Richtung, was von der Numerik bestätigt wird (s. Abb.6.4). Variation von $\kappa_{0}$ führt zu vermiedenen Bandkreuzungen und um $\kappa_{0}=\pi \sigma_{q}$ entstehen so Niveaus im $q$-Spektrum. Um sicherzustellen, dass es sich wirklich um Niveaus handelt und nicht um schmale Bänder, wurde der Ausschnitt des Spektrums zu einem größeren Approximanden berechnet (s. Vergrößerungen Abb. 6.3). Ändert man also $\kappa_{0}$ von 0 nach $\pi \sigma_{q}$, so sollte man im Wellenpaket einen Übergang von lokalisiert zu ausgebreitet sehen. Dies findet man auch wie Abb. 6.4b zeigt. Die Ausbreitung in der Husimi-Darstellung erfolgt wieder in der Form eines Kreuzes. Der Anteil des Wellenpaketes, welches sich in $q$-Richtung ausbreitet, hat ein größeres Gewicht als der Zweig in $p$-Richtung, was durch die größere Anzahl von Bändern im $q$-Spektrum verursacht wird. Deutlich zu sehen sind auch die unterschiedlichen Lokalisierungslängen in $p$ - und $q$-Richtung. 

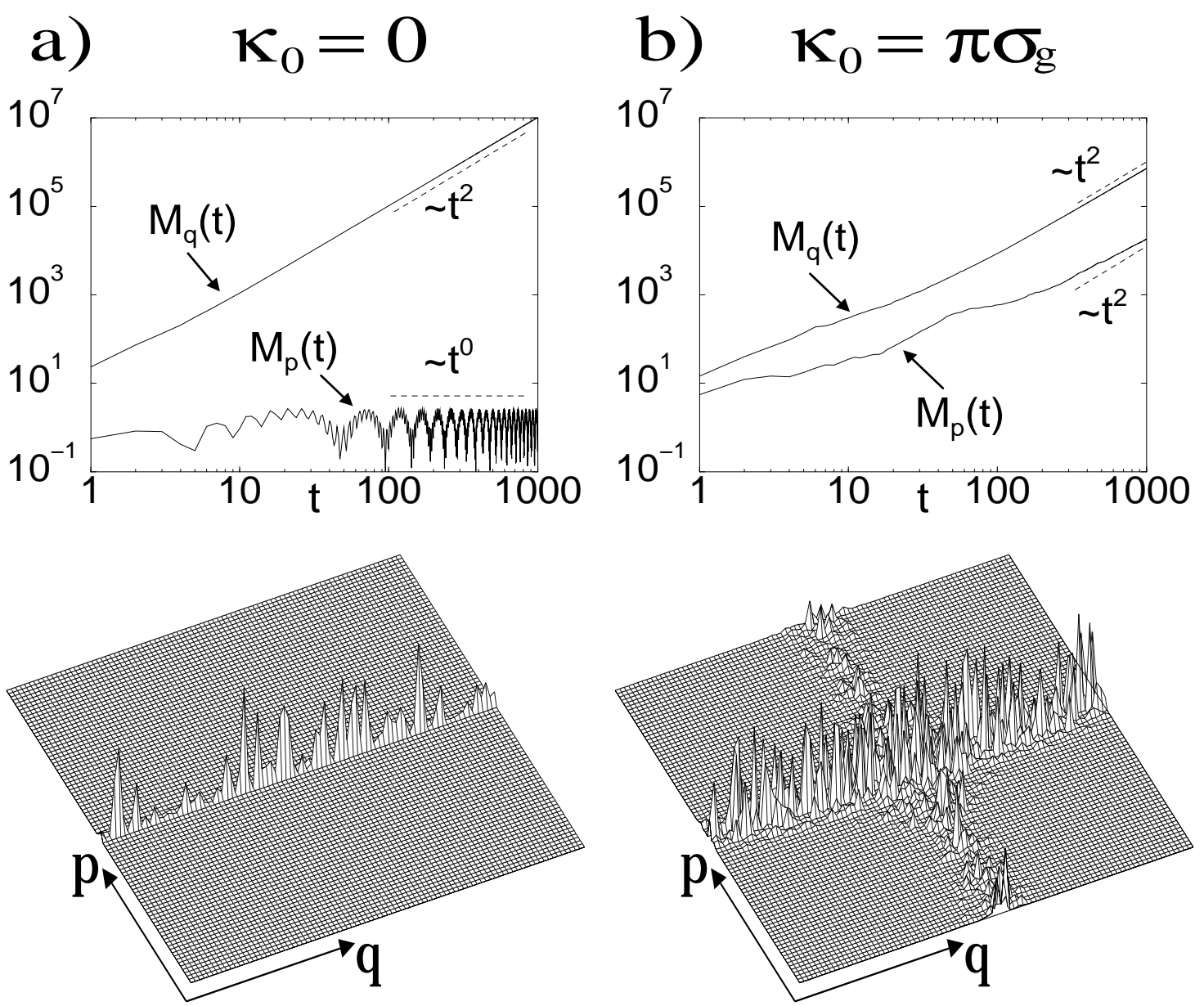

Abbildung 6.4: Die Varianzen $M_{p}(t)$ und $M_{q}(t)$ und die zugehörige Wellenfunktion nach 100 Kicks in Husimi-Darstellung zu den Parametern $K=2$ und $L=4$ und $\hbar_{\text {eff }}=2 \pi /\left(7+\sigma_{q}\right)$ sind gezeigt. Für die Husimi-Darstellung wurden 89 Einheitzellen in $p$ - und $q$-Richtung verwendet. a) Für $\kappa_{0}=0$ breitet sich das Wellenpaket ballistisch in $q$-Richtung aus und lokalisiert in $p$-Richtung. b) Für $\kappa_{0}=\pi \sigma_{q}$ findet man in beide Richtungen ballistische Ausbreitung des Wellenpakets. In Husimi-Darstellung hat das Wellenpaket die Form eines Kreuzes.

\subsection{Zusammenfassung}

In diesem Kapitel wurde die Dynamik von Bloch-Elektronen im magnetischen Feld und zusätzlichem zeitlich oszillierendem elektrischen Feld untersucht und mit spektrale Eigenschaften in Verbindung gebracht. Dadurch konnte ein kürzlich beobachteter Metall-IsolatorÜbergang, verursacht durch eine Variation der elektrischen Feldstärke, erklärt werden. Eine besonders anschauliche Darstellung der Dynamik erlaubt die Projektion des Wellenpakets auf den Phasenraum in der Husimi-Darstellung. Und es wurde gezeigt, dass im Allgemeinen die Ausbreitung in Form eines Kreuzes geschieht. 


\section{Kapitel 7}

\section{Superballistischer Transport}

Die Zeitentwicklung eines Wellenpakets auf einem eindimensionalen (1D) oder quasi-1D Gitter wird durch die Schrödingergleichung

$$
\imath \frac{d c_{n}(t)}{d t}=\sum_{m=n-b}^{m=n+b} H_{n m} c_{m}(t)
$$

beschrieben, wobei $c_{n}(t)$ die Wahrscheinlichkeitsamplitude des Elektrons auf dem $n$-ten Platz ist. Die Anzahl der Kanäle ist durch $b$ gegeben und $H_{n m}$ ist der tight-binding Hamilton-Operator. Die Zeitentwicklung eines Wellenpakets ist eng verknüpft mit den Transporteigenschaften von elektronischen Systemen. Ist das System translationsinvariant, so sind die Eigenfunktionen ebene Wellen und ein ursprünglich lokalisiertes Wellenpaket breitet sich ballistisch aus. Im Gegensatz hierzu ist seit den Arbeiten von Anderson [118] bekannt, dass der Transport durch Unordnung unterdrückt wird, die zu Lokalisierung führt. Speziell im eindimensionalen (1D) Fall erzwingt schon eine beliebig kleine Unordnungsstärke lokalisierte Eigenzustände [119,120], und die Ausbreitung eines Wellenpakets saturiert. In höheren Dimensionen findet man einen Übergang von lokalisierten zu delokalisierten Zuständen und diffusives oder subdiffusives Anwachsen des Wellenpakets kann beobachtet werden. Darüber hinaus findet man bei quasi-periodischen Systemen mit fraktalen Spektren und Eigenfunktionen anomale Diffusionsprozesse [121-123].

Eine globale Charakterisierung der Dynamik eines Wellenpakets ist durch die Varianz

$$
M(t) \equiv \sum_{n} n^{2}\left|c_{n}(t)\right|^{2} \propto t^{\nu}
$$

gegeben. Die Zeitentwicklung gibt eine quantitative Beschreibung der Dynamik: $\nu=0$ zeigt Lokalisierung, $\nu=1$ Diffusion, $\nu=2$ ballistischen Transport und $\nu \in(0,2)$ zeigt anomale Diffusion an. Guarneri und Schulz-Baldes konnten zeigen, dass es eine obere Grenze $M(t) \leq A t^{2}$ für das Anwachsen eines Wellenpakets gibt, wobei die konstante $A$ vom betrachteten System abhängt [124,125]. Asymptotisch kann die Varianz eines Wellenpakets 


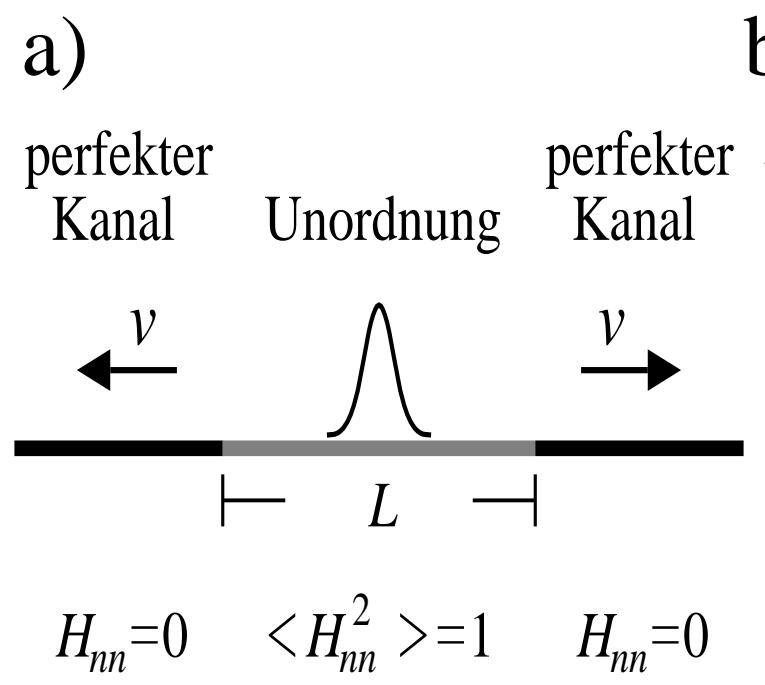

b)

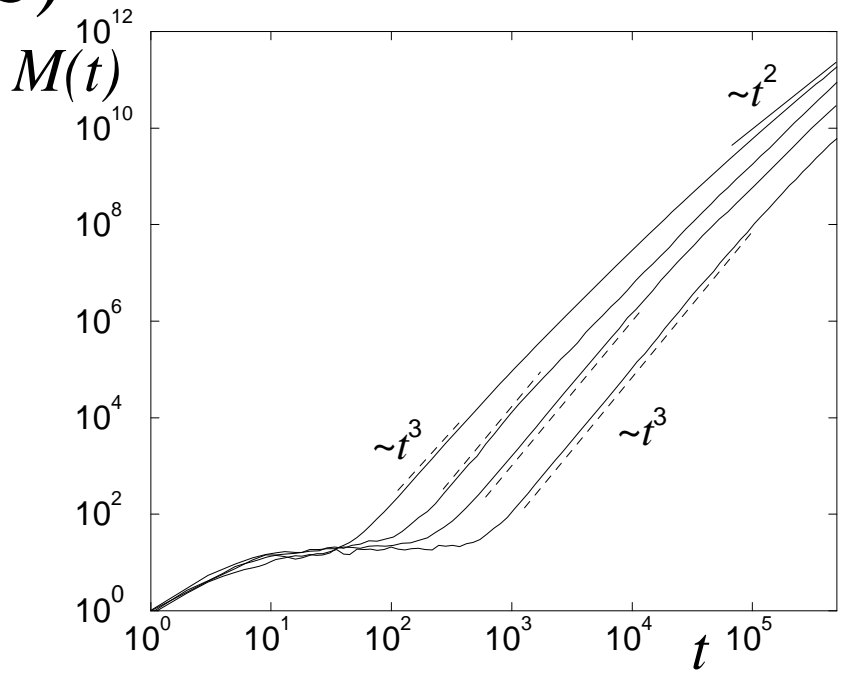

Abbildung 7.1: a) Schematischer Aufbau des Anderson-Modells mit Kanälen. An ein Stück Unordnung der Länge $L$ sind an beiden Seiten perfekte Kanäle angebracht. Das $\delta$-förmiges Wellenpaket wird mitten im ungeordneten Teil gestartet. b) Varianz $M(t)$ eines Wellenpaketes für das 1D Anderson-Modell mit perfekten Kanälen. Die vier Kurven unterscheiden sich in der Länge des ungeordneten Teils ( $L=50,70,80$ und 100 von links nach rechts). Die gestrichelten Linien geben den Bereich an, über den die Varianz superballistisch anwächst. Asymptotisch zerfließt das Wellenpaket ballistisch.

demnach nicht stärker als ballistisch anwachsen, aber auch für endliche Zeiten wurden nur Exponenten $\nu \leq 2$ beobachtet. Es ist eine interessante Frage, ob es (konservative) Systeme gibt, die für endliche Zeiten einen Anstieg der Varianz mit $\nu>2$ zeigen [41].

\subsection{Superballistischer Transport}

Es soll nun gezeigt werden, dass eine sehr allgemeine Klasse von Systemen superballistische Ausbreitung von Wellenpaketen, charakterisiert durch $\nu>2$, aufweist. Die Systeme bestehen aus einem ungeordneten Bereich der Länge $L$, an dessen beiden Enden jeweils ein perfekter Kanal angebracht ist (s. Abb.7.1a). In Abb.7.1b ist der zeitliche Verlauf der Varianz $M(t)$ für ein 1D Anderson-Modell gezeigt. Das Intervall, in dem die Varianz superballistische anwächst, hängt dabei von der Länge des ungeordneten Bereichs ab. Überraschenderweise vergrößert sich - auf einer logarithmischen Skala - die Länge des Intervalls, wenn die Unordnung zunimmt, d.h. die Länge $L$ des ungeordneten Bereichs anwächst. Es wird im Folgenden gezeigt, dass dieses unerwartete Verhalten mit dem Zerfall der Aufenthaltswahrscheinlichkeit eines Elektrons in der ungeordneten Region zusammenhängt. 
Dieser Zerfall wird durch eine Rate $\Gamma$ beschrieben. Sie gibt an, wann das superballistische Verhalten anfängt und endet

$$
t_{\text {on }} \sim\left(\frac{1}{\Gamma}\right)^{1 / \nu} \quad \text { and } \quad t_{\text {off }} \sim \frac{1}{\Gamma} .
$$

Die unterschiedliche $\Gamma$-Abhängigkeit von $t_{\text {on }}$ und $t_{\text {off }}$ stellt dabei sicher, dass der Bereich, über den die Varianz superballistisch anwächst, beliebig groß werden kann, wenn nur $\Gamma$ klein genug gewählt wird.

\subsection{Ein einfaches Modell}

Es soll zunächst ein einfaches probabilistisches Modell vorgestellt werden, das den kubischen Anstieg in der Varianz erklärt. Dazu wird der ungeordnete Teil durch eine Punktquelle ersetzt, und alles, was von der Quelle emittiert wird, entfernt sich mit konstanter Geschwindigkeit $v$ von der Quelle. Zunächst befindet sich das Elektron in der Quelle. Die Emission wird durch eine Rate $\Gamma$ beschrieben, so dass die Wahrscheinlichkeit, dass sich das Elektron in der Quelle befindet, exponentiell abnimmt

$$
P(t)=\exp (-\Gamma t)
$$

Der Wahrscheinlichkeitsfluss zur Zeit $t$ aus der Quelle ist durch $-\dot{P}(t)$ gegeben. Die Varianz $M_{\mathrm{PS}}(t)$ des Punktquellen-Modells berechnet sich dann gemäß

$$
M_{\mathrm{PS}}(t)=\int_{0}^{\infty} d x x^{2} \int_{0}^{t} d t^{\prime}\left(-\dot{P}\left(t^{\prime}\right)\right) \delta\left(x-v t^{\prime}\right)
$$

Setzt man nun Gl. (7.4) für $P(t)$ ein, so erhält man

$$
M_{\mathrm{PS}}(t)=v^{2} \Gamma \int_{0}^{t} d t^{\prime} e^{-\Gamma t^{\prime}} t^{\prime 2}
$$

Und nach Integration folgt

$$
M_{\mathrm{PS}}(t)=v^{2}\left(t^{2}-\frac{2}{\Gamma} t+\frac{2}{\Gamma^{2}}-\frac{2}{\Gamma^{2}} e^{-\Gamma t}\right) .
$$

Wie zu erwarten war, wächst die Varianz für große Zeiten quadratisch gemäß $M_{\mathrm{PS}}(t) \sim v^{2} t^{2}$ an. Entwickelt man die Exponentialfunktion in Gl. (7.7)), so erhält man

$$
M_{\mathrm{PS}}(t)=v^{2} \Gamma\left(\frac{1}{3} t^{3}-\frac{\Gamma}{12} t^{4}+\ldots\right)
$$


Ist $t<1 / \Gamma$, so dominiert der kubische Term alle höheren Ordnungen. Das PunktquellenModell liefert also ein kubisches Anwachsen der Varianz von $t_{\text {on }}=0$ bis zur Zeit $t_{\text {off }} \approx 1 / \Gamma$.

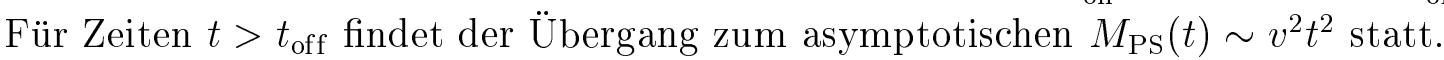

Intuitiv begründet sich das kubische Verhalten folgendermaßen. Für Zeiten $t<1 / \Gamma$ fällt die Wahrscheinlichkeit das Elektron in der Quelle zu finden, linear gemäß $P(t) \approx 1-\Gamma t$ ab. Während dieser Zeit wächst die Norm außerhalb der Quelle linear an. Da alles, was von der Quelle emittiert wird, sich mit konstanter Geschwindigkeit von der Quelle entfernt, führt das lineare Anwachsen der Norm und der ballistische Transport der Kanäle zum kubischen Anstieg der Varianz.

Ein realistischeres Modell muss die endliche Ausdehnung des ungeordneten Bereichs berücksichtigen. Damit verbunden ist die Zeit $\tau$, die das Wellenpaket benötigt, um von der Mitte des ungeordneten Bereichs zu den Kanälen zu gelangen, so dass die Norm in den Kanälen linear anwächst. Die Varianz setzt sich additiv aus zwei Teilen zusammen: der internen Varianz $M_{\text {int }}(t)$ für den ungeordneten Bereich und der externen Varianz $M_{\text {ext }}(t)$, die die Ausbreitung der Wellenfunktion in den Kanäle beschreibt. Die externe Varianz berechnet sich analog zum Punkquellen-Modell nach

$$
M_{\mathrm{ext}}(t)=\int_{L}^{\infty} d x x^{2} \int_{\tau}^{t} d t^{\prime}\left(-\dot{P}\left(t^{\prime}\right)\right) \delta\left(x-L-v\left(t^{\prime}-\tau\right)\right) .
$$

Unter den drei Bedingungen (i) $t \gg \tau$, (ii) $v t \gg L$ und (iii) $M_{\text {ext }}(t)>M_{\text {int }}(t)$ reduziert sich Gl. (7.9) auf das Punktquellen-Modell. Diese Bedingungen legen die Zeit $t_{\text {on }}$ fest. Bedingungen (i) und (ii) liefern die Zeiten $\tau$ und $L / v$. Die interne Varianz $M_{\text {int }}(t)$ ist durch $M_{\text {int }}^{\max } \leq L^{2}$ beschränkt. Diese Bedingung zusammen mit $M_{\text {ext }}(t) \approx v^{2} \Gamma / 3 t^{3}$ für $t<t_{\text {off }}$ ergibt die Zeitskala $\left[M_{\text {int }}^{\max } /\left(v^{2} \Gamma\right)\right]^{1 / 3}$. Das Maximum dieser drei Zeiten markiert den Anfang des kubischen Anstiegs

$$
t_{\text {on }}=\max \left\{\tau, \frac{L}{v},\left(\frac{M_{\text {int }}^{\max }}{v^{2} \Gamma}\right)^{1 / 3}\right\} .
$$

Ist $\Gamma$ hinreichend klein, so bestimmt der letzte Term das Maximum. Nach Gl. (7.3) skaliert das Verhältnis $t_{\text {off }} / t_{\text {on }}$ wie $\Gamma^{-2 / 3}$ und kann daher beliebig anwachsen, wenn $\Gamma$ nur klein genug ist.

\subsection{Numerische Behandlung}

Die Vorhersagen des obigen Modells sollen nun numerisch überprüft werden. Dies soll anhand eines 1D Anderson-Modells und quasi-1D Bandzufallsmatrix-Modells (BRM) erfolgen. Beide Systeme sind spezielle Realisierungen des tight-binding Hamilton-Operators aus Gl. (7.1), weshalb zunächst auf die gewählte numerische Methode zur Lösung der Differentialgleichung eingegangen werden soll. Dabei ist es wichtig, ein numerisch effizientes 


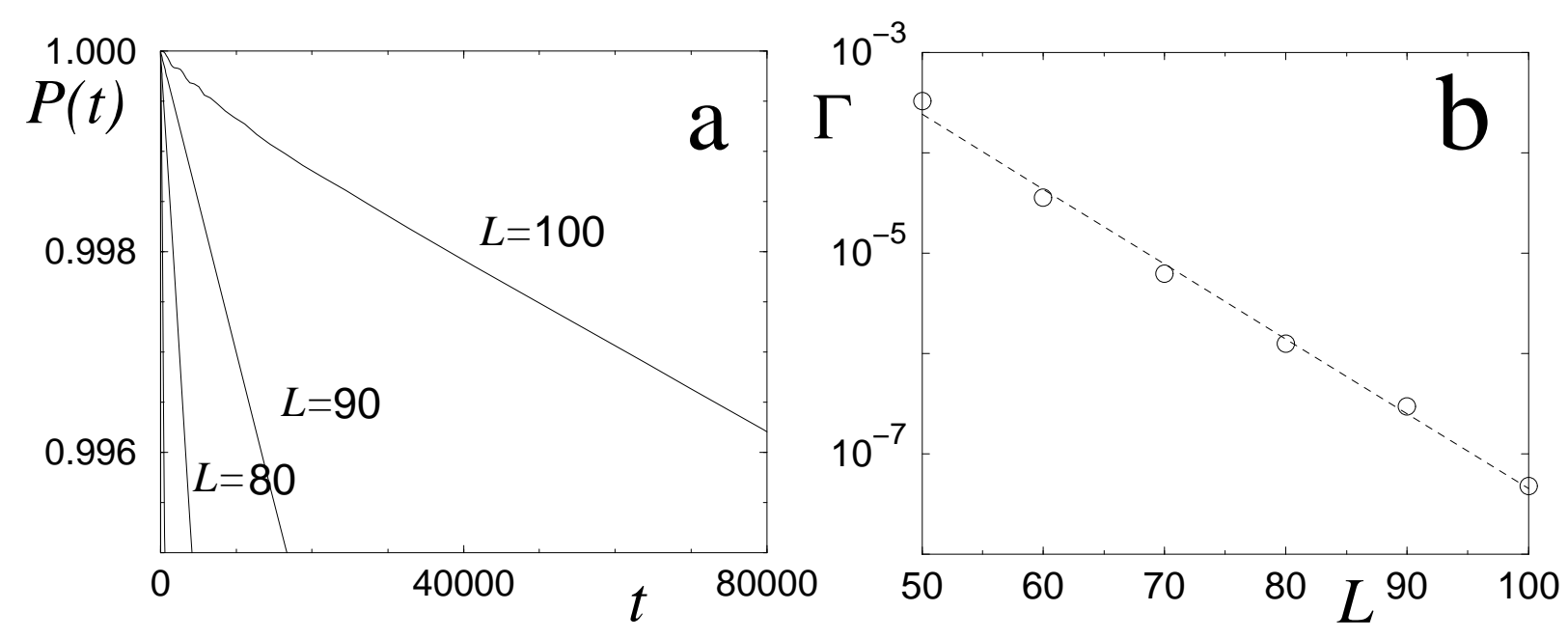

Abbildung 7.2: a) Die Norm des Wellenpakets innerhalb des ungeordneten Bereichs für das 1D Anderson-Modell als Funktion der Zeit. Der lineare Zerfall wird durch die Zerfallsrate $\Gamma$ beschrieben. b) Die Zerfallsrate $\Gamma$ wird exponentiell kleiner mit der Länge $L$ des ungeordneten Bereichs.

und zugleich sehr exaktes Verfahren zu wählen, da zum einen der kubische Anstieg die Behandlung von sehr großen Systemen erzwingt und zum anderen sich kleine Abweichungen in den Schwänzen der Verteilung in der Varianz niederschlagen.

Um die Zeitentwicklung eines Anfangswellenpaketes gemäß der tight-binding-Gleichung Gl. (7.1) zu untersuchen, kann man auf das einfache Runge-Kutter-Verfahren 4. Ordnung zurückgreifen. Dieses hat jedoch den Nachteil, dass die Unitarität des Zeitentwicklungsoperators nicht ausgenutzt wird. Man muss daher sehr kleine Zeitinkrementen $\delta t$ verwenden um sicherzustellen, dass die Norm des Wellenpakets gut genug erhalten bleibt. Eine angemessenere Integration der tight-binding-Gleichung bietet das Cayley-Verfahren. Dabei wird der Zeitentwicklungsoperator für einen kleinen Schritt $\delta t$ durch die Cayley-Form

$$
\mathcal{U}(\delta t)=\exp (-\imath \mathcal{H} \delta t) \approx \frac{1-\imath \mathcal{H} \delta t / 2}{1+\imath \mathcal{H} \delta t / 2}
$$

angenähert. Ist der Zustand des Systems $c_{n}(t)$ zur Zeit $t$ bekannt, so erhält man das Wellenpaket $c_{n}(t+\delta t)$ als Lösung des Gleichungssystems

$$
\sum_{m=-N}^{N}(1+\imath \mathcal{H} \delta t / 2)_{n m} c_{m}(t+\delta t)=\sum_{m=-N}^{N}(1-\imath \mathcal{H} \delta t / 2)_{n m} c_{m}(t)
$$

Die Dimension der Matrix $N$ muss dabei so groß gewählt werden, dass das Wellenpaket für die gewünschten Zeiten nicht an die Ränder gelangt. Bei der numerischen Lösung des Gleichungssystems nutzt man zweckmäßigerweise die Bandbreite $b$ der Matrizen $1 \pm \imath \mathcal{H} \delta t / 2$ aus. Der Rechenaufwand skaliert dann genau wie beim Runge-Kutter-Verfahren mit 


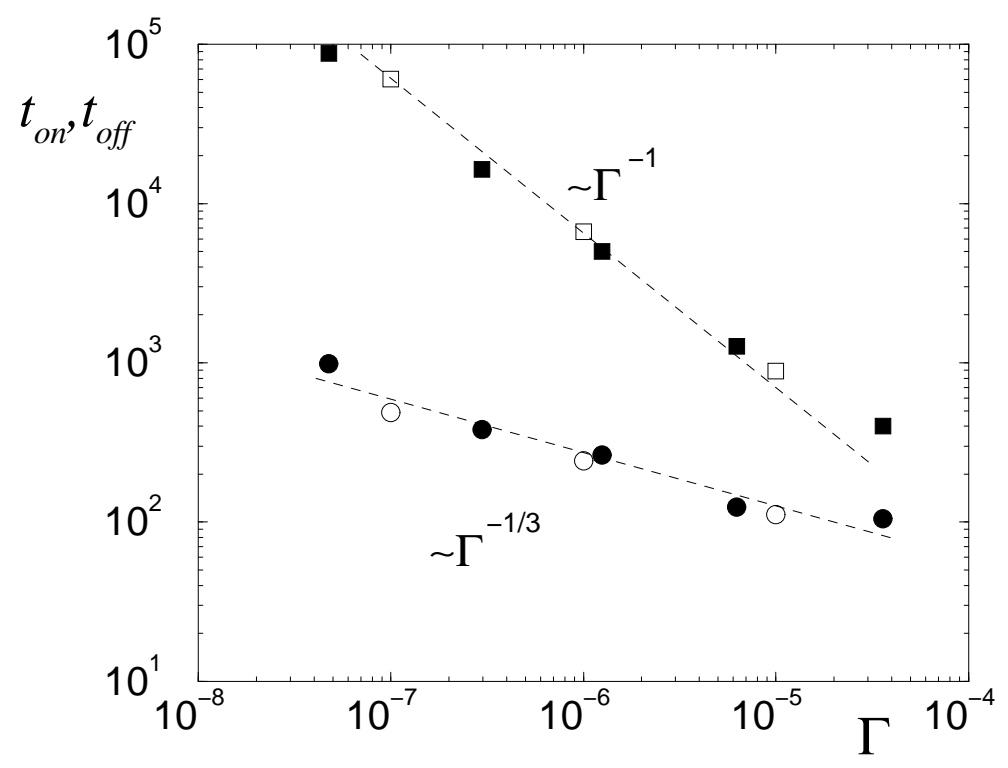

Abbildung 7.3: Abhängigkeit der Zeiten $t_{\text {on }}$ (Quadrate) und $t_{\text {off }}$ (Kreise) von der Zerfallsrate $\Gamma$ für das 1D Anderson-Modell (gefüllte Symbole) und dem BRM-Modell (leere Symbole). Die Daten zum BRM-Modell wurden verschoben, um so den Einfluss der unterschiedlichen Vorfaktoren beider Modelle zu kompensieren.

$b N$. Der große Vorteil des Cayley-Verfahrens liegt jedoch darin begründet, dass man im Vergleich zum Runge-Kutter-Verfahren wesentlich größere Zeitinkrementen $\delta t$ verwenden kann. Liegen erstere typischerweise bei einigen $10^{-4}$, so wurde in Ref. [126] für BRMModelle gezeigt, dass $\delta t \leq 1 / \sqrt{b}$ verlässliche Ergebnisse liefert. Das Cayley-Verfahren ist demnach sehr gut für die numerische Behandlung der obigen Systeme geeignet und erlaubt Systemgrößen von $N>10^{7}$.

\subsubsection{Anderson-Modell}

Zunächst sollen die Resultate für das 1D Anderson-Modell vorgestellt werden. Der HamiltonOperator Gl. (7.1) ist tridiagonal und die Nebendiagonalelemente $H_{n n+1}=H_{n+1 n}$ sind auf eins gesetzt. Innerhalb einer Region der Länge $L$ werden die Diagonalelemente nach einer Gauß-Verteilung mit Varianz eins gewählt. Außerhalb der Region verschwinden die Diagonalelemente. Der einzige freie Parameter ist die Länge des ungeordneten Bereichs. Für alle betrachteten Längen $L=50, \ldots, 100$ gilt $L>l_{\infty}$. Dabei ist $l_{\infty}$ die maximale Lokalisierungslänge, die man erhielte, wenn auf der Diagonalen überall Zufallszahlen stünden. Für alle Längen wird ein ursprünglich $\delta$-förmiges Wellenpaket in der Mitte des ungeordneten Bereichs gestartet. In Abb.7.1 sind die Varianzen für vier verschiedene Längen gezeigt. Dabei wurde über zehn Realisierungen der Unordnung gemittelt. Zwar zeigt jede einzelne Realisierung auch das kubische Anwachsen in der Varianz, jedoch kann der Startzeitpunkt 
mit der speziellen Realisierung der Unordnung variieren. Für kleine Zeiten breitet sich das Wellenpaket zunächst ballistisch aus, bis die Varianz bei $M_{\text {int }}(t) \approx l_{\infty}^{2}$ saturiert. Dann setzt der kubische Anstieg ein, bevor sich das Wellenpaket für sehr große Zeiten wieder ballistisch ausbreitet. Das überraschende Ergebnis, dass eine Vergrößerung des ungeordneten Bereichs den kubischen Anstieg in der Varianz verlängert, erklärt sich folgendermaßen: Die Wellenfunktionen sind innerhalb des ungeordneten Bereichs exponentiell lokalisiert. Störungstheoretisch liefert die Aufenthaltswahrscheinlichkeit der Eigenfunktionen an der Schnittstelle zwischen Unordnung und Kanal die Zerfallsbreite der Eigenfunktion. Wird nun bei festgehaltener Lokalisierungslänge $l_{\infty}$ der ungeordnete Bereich vergrößert, so verkleinert sich die Zerfallsbreite exponentiell. Man erwartet daher, dass $\Gamma$ exponentiell von der Länge $L$ abhängt. Um dies zu überprüfen, wurde für sechs verschiedene Längen $\Gamma$ bestimmt. Dazu wurde der Abfall der Norm innerhalb des ungeordneten Bereichs untersucht. Aus dem linearen Abfall für kleine Zeiten kann man $\Gamma$ bestimmen (s. Abb. 7.2a). Wie man in Abb. 7.2b sieht, verringert sich $\Gamma$ exponentiell mit $L$. Bleibt noch zu überprüfen, wie $t_{\text {on }}$ und $t_{\text {off }}$ mit $\Gamma$ skalieren. Um die Zeitskalen zu bestimmen, wurde für jede Länge $L$ an die Varianzkurve $M(t)$ das kubische Verhalten gefittet und die Zeit bestimmt, zu denen der Fit mehr als 10\% von der Varianz abweicht. Die so ermittelten Zeiten für $t_{\text {on }}$ und $t_{\text {off }}$ sind in Abb. 7.3 zusammengestellt und bestätigen gut das Verhalten gemäß Gl. (7.3).

\subsubsection{BRM-Modell}

Eine realistische Beschreibung von Quantendrähten bietet das BRM-Modell [127-129]. Die Hamilton-Matrix $H_{n m}$ ist reell und die Einträge sind nur von null verschieden innerhalb eines Streifens der Breite $b$ um die Diagonale $(|n-m| \leq b)$. Die Bandbreite $b$ legt fest, wie viele Plätze miteinander koppeln, oder in einer quasi-1D Interpretation gibt $b$ die Anzahl der transversalen Moden an $[127,128]$. Die Matrixelemente sind gaußsch verteilt mit der Varianz eins in einem Bereich der Länge $L$. Außerhalb des Bereichs werden alle Elemente auf eins gesetzt. Die Matrixelemente, die den ungeordneten Bereich mit den Kanälen koppeln, sind ebenfalls gaußsch verteilt, jedoch mit der Varianz $T$ (s. Abb. 7.4a). Die Koppelungsstärke $T$ bestimmt die Zerfallsbreite $\Gamma \sim T$ [130]. Ein ursprünglich am Site $n_{0}=0 \delta$-förmiges Startwellenpaket breitet sich zunächst ballistisch aus, denn es gilt für kleine Zeiten $\delta t$

$$
M(\delta t) \approx \sum_{n} n^{2}|1+\imath H \delta t|_{n n_{0}}^{2}=\sum_{n=-b}^{b} n^{2} H_{n 0}^{2}=v \delta t
$$

Dabei hängt die Geschwindigkeit $V$ gemäß $v \approx \sum_{n=-b}^{b} n^{2}=b(b+1)(2 b+1) / 3 \sim b^{3}$ von der Bandbreite $b$ ab. Wird die Breite des Wellenpakets $\sqrt{M(t)}$ vergleichbar mit $b$, so endet das ballistische Verhalten und die Varianz wächst diffusiv an. Dieser Übergang liegt bei der Zeit $t_{b} \approx 1 / \sqrt{b}$. Im Gegensatz zum Anderson-Modell existiert für das BRM-Modell ein Bereich, in dem die Varianz diffusiv anwächst. Dies liegt darin begründet, dass die mittlere 

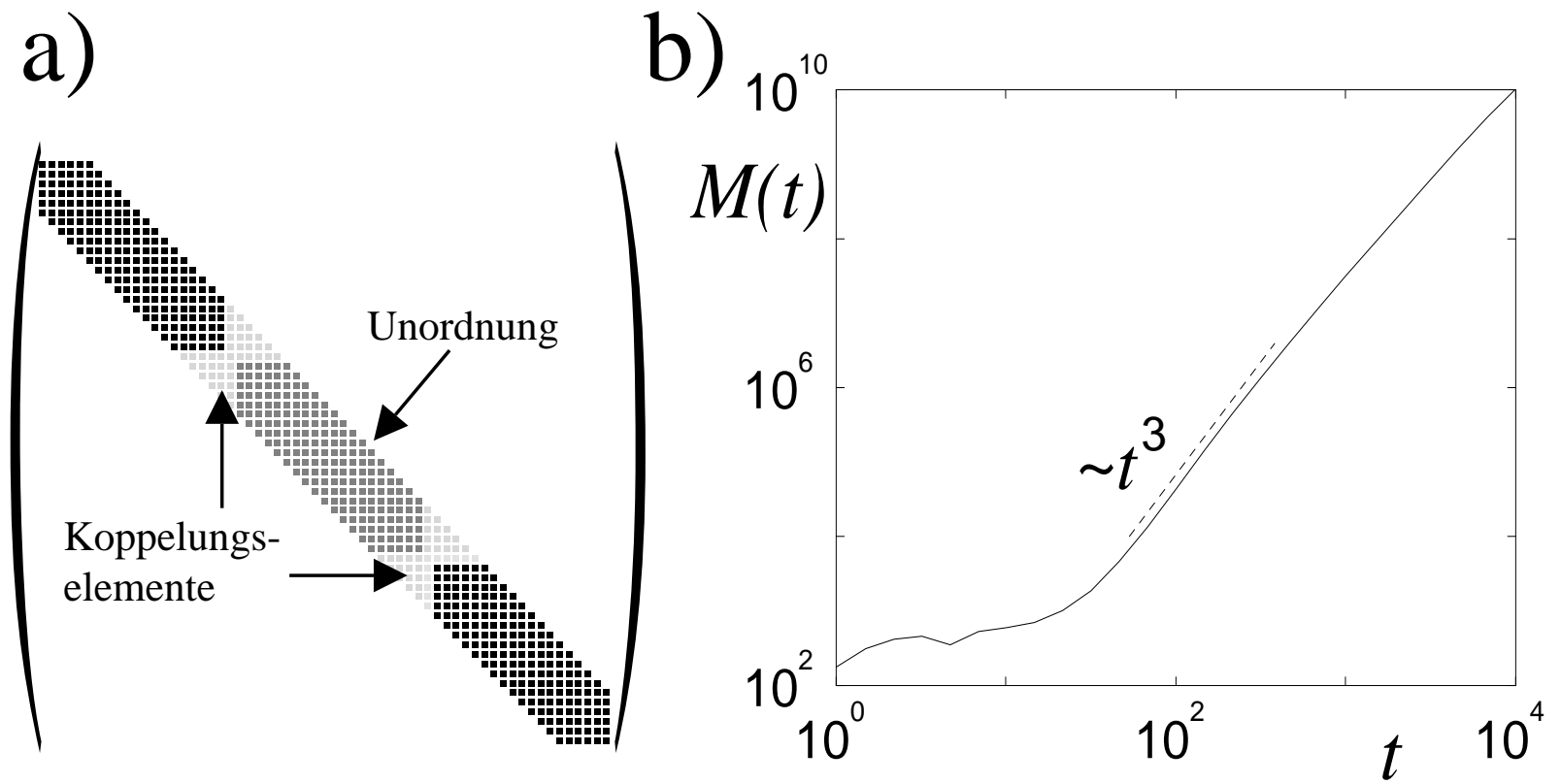

Abbildung 7.4: a) Schematische Darstellung eines Ausschnitts des BRM Hamilton-Operators $H_{n m}$ mit Bandbreite $b=5$. Der Bereich mit Unordnung (Varianz 1) ist mit zwei perfekten Kanälen (konstant 1) über Koppelungselemente mit der Varianz $T$ verbunden. b) Verlauf der Varianz $M(t)$ für das BRM-Modell mit $b=10, L=100$ und $T=0.001$.

freie Weglänge $l_{m} \sim b$ und die Lokalisierungslänge $l_{\infty} \sim b^{2}$ unterschiedlich mit der Bandbreite $b$ skalieren. Die Diffusionskonstante hängt dabei von der Bandbreite wie $D \sim b^{2.5}$ ab. Zur Zeit $t_{D} \sim b^{1.5}$, Thoulous-Zeit, endet die Diffusion und Lokalisierung setzt ein, wenn die Bandmatrix überall zufällige Einträge enthält [128]. Die Länge des ungeordneten Bereichs für das BRM-Modell mit Kanälen soll nun so gewählt werden, dass das Wellenpaket diffusiv die Ränder der Kanäle erreicht, d.h. $L<l_{\infty}$. Auch das BRM-Modell zeigt das kubische Anwachsen der Varianz (s. Abb. 7.4b). Die Variation der Koppelungsstärke verändert den Anfangs- und Endpunkt. Wie beim Anderson-Modell wurden die Zeiten $t_{\text {on }}$ und $t_{\text {off }}$ für verschiedene Koppelungsstärken bestimmt. Diese bestätigen sehr gut das Skalierungsverhalten gemäß Gl. (7.3), wie Abb. 7.3 zeigt.

\subsubsection{Fibonacci-Modell}

Zum Abschluß soll noch dargestellt werden, wie man beliebige Exponenten $\nu$ zwischen 2 und 3 erhält. Der Einfachheit halber soll dies an einem 1D System geschehen. Für quasiperiodische Systeme, wie z.B. das Harper- oder das Fibonacci-Modell, ist bekannt, dass sich Wellenpakete mit Exponenten $0<\mu<2$ ausbreiten (anomale Diffusion). Der Exponent $\mu$ wird dabei in guter Näherung durch die fraktale Dimension des Spektrums $D_{0}$ gemäß $\mu=2 D_{0}$ 

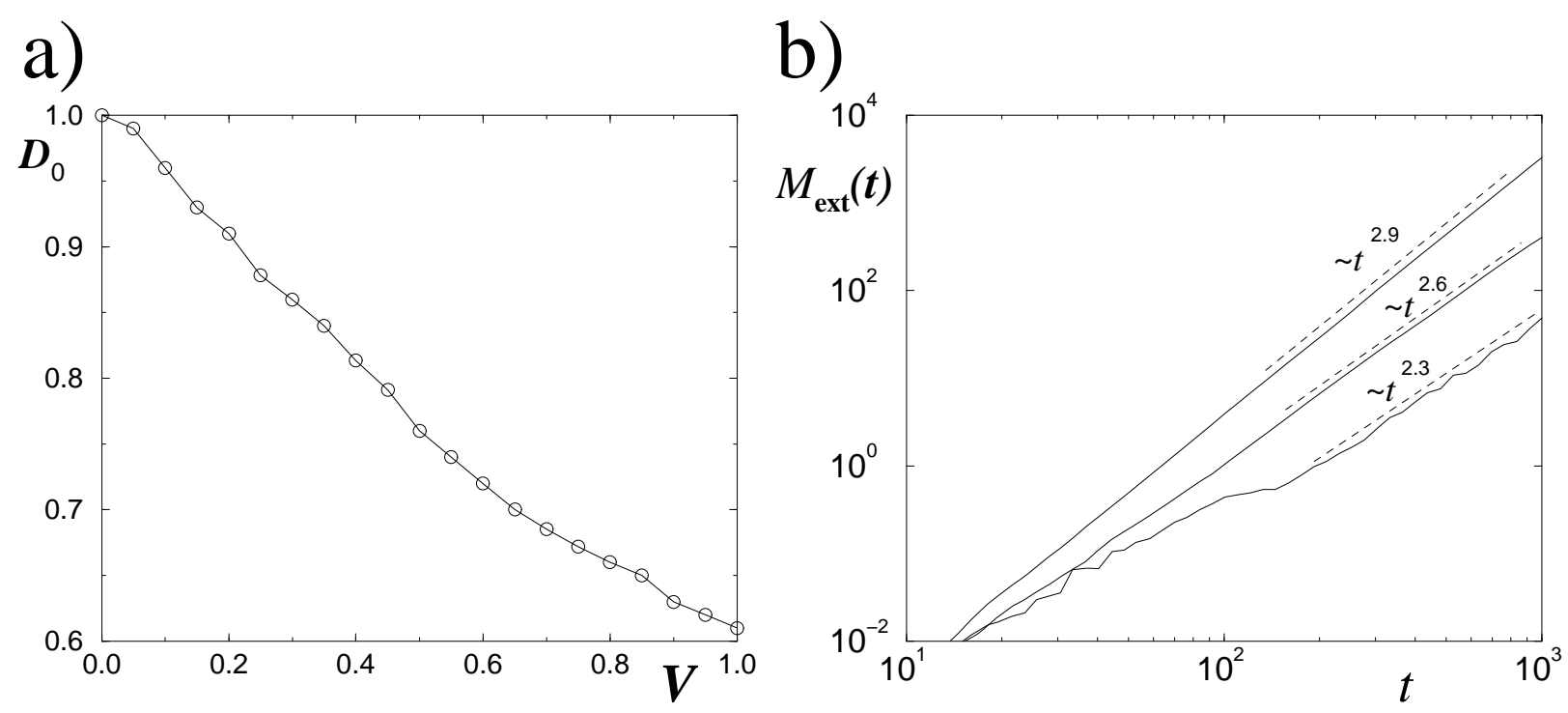

Abbildung 7.5: a) Abhängigkeit der fraktalen Dimension $D_{0}$ für das Fibonacci-Modell von der Potentialstärke $V$. b) Die externe Varianz $M_{\text {ext }}(t)$ für einen ungeordneten Bereich der Länge $L=50$ eingebettet in das Fibonacci-Modell für die drei Potentialstärken $V=0.1,0.4$ und 0.7.

bestimmt $[117,131]$. Bettet man nun einen ungeordneten Bereich der Länge $L$ in ein quasiperiodisches System ein und vollzieht die analogen Schritte, die zu Gl. (7.8) führten, so findet man, dass die Varianz für Zeiten $\Gamma t^{\mu}<1$ mit dem Exponenten $\nu=\mu+1=2 D_{0}+1$ anwächst. Ein System, an dem man dieses Verhalten gut studieren kann, ist das FibonacciModell $[132,133]$. Die Nebendiagonalelemente des Hamilton-Operators haben alle den Wert eins. Außerhalb des ungeordneten Bereichs, können die Diagonalelemente die Werte $\pm V$ annehmen, wobei die Fibonacci-Sequenz festlegt, wo das Potential positiv oder negativ ist. Die fraktale Dimension $D_{0}$ kann durch die Potentialstärke $V$ eingestellt werden (s. Abb. 7.5a). Diese erlaubt es, die Beziehung $\nu=\mu+1=2 D_{0}+1$ zu überprüfen. In Abb. 7.5b ist die externe Varianz $M_{\text {ext }}(t)$ für drei unterschiedliche Potentialstärken gezeigt. Das Verhalten wird gut durch die zu erwartenden Exponenten beschrieben.

\subsection{Zusammenfassung}

In diesem Kapitel wurde gezeigt, dass die Varianz von Wellenpaketen für eine Klasse von konservativen Systemen schneller als ballistisch anwachsen kann. Die Untersuchungen wurden an 1D und quasi-1D Systemen durchgeführ, die aus einem ungeordneten Teil bestehen, an dem zwei Kanäle angebracht sind. Ein probabilistisches Modell ermöglicht zum einen ein qualitatives Verständnis und zum andern eine quantitative Vorhersage zur Dauer des superballistischen Verhaltens, die gut von den Simulationen bestätigt wird. 


\section{Kapitel 8}

\section{Zusammenfassung}

Im Rahmen dieser Arbeit werden Transporteigenschaften niedrigdimensionaler HamiltonSysteme unter besonderer Berücksichtigung von Systemen mit gemischtem Phasenraum untersucht. Im Zusammenhang mit der Frage nach dem Einfluss der klassischen chaotischen Dynamik auf das zugehörige Quantensystem wird zunächst die klassische Dynamik in gemischten Systemen genauer betrachtet. Dabei wird gezeigt, dass selbst dann, wenn der Phasenraum durch eine exakt selbst-ähnliche Struktur dominiert wird, diese nicht die asymptotische Dynamik bestimmt. Die quantenmechanische Untersuchung gemischter Systeme führt zu einer neuen Klasse von Eigenfunktionen - den hierarchischen Eigenzuständen -, deren Existenz durch die klassische Phasenraumstruktur begründet wird. Daran anschließend werden Leitwertfluktuationen generischer Billards untersucht. Der Ursprung der kürzlich beobachteten und bis dato unverstandenen isolierten Resonanzen wird geklärt. Es wird eine einheitliche Theorie für Leitwertfluktuationen in typischen Hamilton-Systemen entwickelt, die den Widerspruch zwischen fraktalen Leitwertfluktuationen und isolierten Resonanzen auflöst. Weiterhin wird die Dynamik von Bloch-Elektronen im Magnetfeld studiert und ein beobachteter Metall-Isolator-Übergang mit vermiedenen Bandkreuzungen erklärt. Die Ausbreitung eines Elektron erfolgt dabei im Allgemeinen in der Form eines Kreuzes. Schließlich wird gezeigt, dass die Varianz eines Wellenpakets stärker als ballistisch anwachsen kann, wobei die Dauer dieser superballistischen Ausbreitung beliebig groß werden kann.

Im Einzelnen beginnt die Arbeit mit einer Einführung in Hamilton-Systeme, wobei der Schwerpunkt auf Systemen mit gemischten Phasenraum liegt. Der hierarchische Aufbau des gemischten Phasenraum mit seiner Abfolge von Inselketten wird diskutiert und der Einfluss der Cantori auf die Dynamik wird besprochen. Die Transporteigenschaften der chaotischen Komponente lassen sich durch ein Markov-Modell beschreiben, das ein qualitatives Verständnis der Dynamik erlaubt.

Charakteristisch für Systeme mit gemischten Phasenraum ist der algebraische Abfall der Verteilung von Aufenthaltszeiten $P_{\mathrm{cl}}(t) \sim t^{-\gamma}$, der durch das Hängenbleiben chaotischer 
Trajektorien an den Inseln verursacht wird. Im Gegensatz zur impliziten Annahme einfacher Renormierungstheorien, die zur Vorhersage des Exponenten $\gamma$ verwendet werden, wird gezeigt, dass die asymptotische Dynamik im Phasenraum nicht durch einen eindimensionalen Pfad im Phasenraum beschrieben wird. Dies gilt selbst dann, wenn der Phasenraum entlang eines Pfades ein exakt selbstähnliches Skalierungsverhalten aufweist, wie man es z.B. am kritischen goldenen Torus beobachtet. Speziell wurde in der Literatur für das Hängenbleiben am kritischen goldenen Torus der Zerfallsexponent $\gamma=3$ vorhergesagt und argumentiert, dass dieser Exponent universell das asymptotische Verhalten der Verteilung beschreibt. Die numerischen Ergebnisse widerlegen jedoch diese Behauptung und zeigen eindeutig, dass Regionen im Phasenraum, die nicht von den eindimensionalen Renormierungstheorien berücksichtig werden, wichtige Beiträge liefern, die für große Zeiten das Verhalten der Verteilung dominieren.

Die quantenmechanische Behandlung der Dynamik im gemischten Phasenraum führt zu einer neuen Klasse von Eigenzuständen, den hierarchischen Zuständen. Obwohl sie als Träger die chaotische Komponente des Phasenraums haben, leben sie nicht gleichverteilt auf dem chaotischen invarianten Bereich, sondern sind in der Nähe der Inseln konzentriert. Ihre Existenz beruht auf der endlichen Auflösung der klassischen Flüsse im Phasenraum durch die Quantenmechanik. So wirken quantenmechanisch partielle Transportbarrieren mit Flüssen kleiner als das Plancksche Wirkungsquantum wie geschlossene Tori. Die Transportbarrieren, deren Fluss gerade gleich $\hbar$ ist, wird als Flussbarriere bezeichnet, und trennt die hierarchischen von den chaotischen Zuständen. Anhand eines einfachen Modells wird der Einfluss der hierarchischen Zustände auf spektrale und Eigenfunktionsstatistiken quantifiziert. Weiterhin wird gezeigt, dass der relative Anteil hierarchischer Zustände im semiklassischen Limes wie $f_{\text {hier }} \sim \hbar_{\text {eff }}^{1-1 / \gamma}$ abnimmt.

Nach den Ausführungen zu spektralen Eigenschaften gemischter Systeme, werden Streueigenschaften gemischter Phasenräume untersucht. Es wird gezeigt, dass die jüngst am Cosinus-Billard beobachteten isolierten Resonanzen im Leitwert Streusignaturen der hierarchischen Zustände des geschlossenen Systems sind. Eine einheitliche Theorie zu Leitwertfluktuationen in typischen Hamilton-Systemen wird aufgestellt und numerisch überprüft. Dadurch wird der Widerspruch zwischen den isolierten Resonanzen und fraktalen Leitwertfluktuationen aufgelöst. Es wird gezeigt, dass im Allgemeinen beide Typen von Leitwertfluktuationen gleichzeitig - jedoch auf unterschiedlichen Energieskalen - auftreten. Die Energieskala, die beide Typen trennt, ist durch die klassische Rate an der Flussbarriere gegeben und skaliert wie $\Delta E^{*} \sim \Delta \hbar_{\text {eff }}^{1 / \gamma-1}$, wobei $\Delta$ der mittleren Niveauabstand ist. $\mathrm{Zu}$ dieser Energie korrespondiert die Zeitskala $t^{*}=h / \Delta E^{*} \sim \hbar / \Delta \hbar_{\mathrm{eff}}^{1-1 / \gamma}$, bis zu der die Quantenmechanik die klassischen Dynamik nachahmt. Dieses Skalierungsverhalten wird mit Hilfe des gekickten Rotators verifiziert. Mit Hilfe eines Quantengraphen-Modells für die klassische Dynamik werden die statistischen Eigenschaften der isolierten Resonanzen untersucht und der Übergang zu fraktalen Fluktuationen demonstriert.

Im nachfolgenden Kapitel werden rein quantenmechanische Eigenschaften der Dynamik von 
Elektronen in einem zweidimensionalen periodischen Potential mit Magnetfeld und zusätzlichem alternierenden elektrischen Feld untersucht. Im Limes großer Feldstärken reduziert sich die Dynamik auf ein doppelt gekicktes System, für das ein Metall-Isolator-Übergang gefunden wurde. Dieser Übergang wird mit vermiedenen Bandkreuzungen erklärt und es wird gezeigt, dass die Ausbreitung eines Wellenpakets im Allgemeinen die Form eines Kreuzes hat.

Zum Abschluss wird die Dynamik von Wellenpaketen für Systeme untersucht, die aus einem ungeordneten Teil bestehen, an dem zwei Kanäle angebracht sind Es wird gezeigt, dass die Varianz von Wellenpaketen stärker als ballistisch anwachsen kann. Die Dauer dieses superballistischen Verhaltens hängt mit der Emissionsrate der Elektronen aus dem ungeordneten Bereich zusammen und kann beliebig lang werden. Ein probabilistisches Modell erklärt dieses unerwartete Verhalten und die Vorhersagen werden an ein- und quasi-eindimensionalen, sowie an quasiperiodischen Modellen verifiziert.

Weitere faszinierende Phänomene sind von zukünftigen theoretischen und experimentellen Untersuchungen des Übergangsbereichs zwischen einer rein quantenmechanischen Beschreibung der mikroskopischen Welt und den Eigenschaften der klassischen Dynamik auf makroskopischen Skalen zu erwarten. 


\section{Literaturverzeichnis}

[1] L. L. Sohn, Nature 394, 11 (1998).

[2] H. Poincaré, Les Méthodes nouvelles de la méchanique céleste (Dover Publ. Inc., New York, 1957).

[3] G. D. Birkhoff, Trans. Am. Math. Soc. 14, 14 (1913).

[4] G. D. Birkhoff, Acta Math. 43, 1 (1920).

[5] G. D. Birkhoff, Memoriae Point. Acad. Sci. Novi Lyncaei 1, 85 (1935).

[6] J. Moser, Nachr. Akad. Wiss. Göttingen Math. Phys. Kl. 2, 1 (1962).

[7] J. Moser, Math. Annalen 169, 136 (1967).

[8] V. I. Arnold, Soviet Math. Dokl. 2, 247 (1961).

[9] V. I. Arnold, Russian Math. Surv. 18, 85 (1963).

[10] V. I. Arnold, Geometrical Methods in the Theory of Ordinary Differential Equations (Springer Verlag, Berlin, Heidelberg, New York, 1983).

[11] S. Aubry, Physica D 7, 240 (1983).

[12] S. Aubry, J. Physique 44, 147 (1983).

[13] J. N. Mather, Topology 21, 457 (1982).

[14] S. Smale, Bull. Amer. Math. Soc. 73, 747 (1967).

[15] B. Chirikov und D. L. Shepelyansky, in Proceedings of the IXth Intern. Conf. on Nonlinear Oscillations (Princeton University Press, Princeton, 1983), No. PPPLTRANS-133.

[16] M. L. Mehta, Random Matrices (Academic Press, Boston, 1991).

[17] O. Bohigas, M. J. Giannoni und C. Schmit, Phys. Rev. Lett.52, 1 (1984). 
[18] A. Einstein, Verh. Deutschen Phys. Ges. Berlin 19, 82 (1917).

[19] L. Brillouin, J. Phys. Radium 7, 353 (1926).

[20] J. B. Keller, Ann. Phys. 4, 180 (1958).

[21] M. C. Gutzwiller, J. Math. Phys. 11, 1791 (1970).

[22] M. C. Gutzwiller, J. Math. Phys. 12, 343 (1971).

[23] M. C. Gutzwiller, Chaos in Classical and Quantum Mechanics (Springer Verlag, Berlin, 1990).

[24] P. A. Lee und A. D. Stone, Phys. Rev. Lett. 55, 1622 (1985).

[25] A. D. Stone und H. Fukuyama, Phys. Rev. B 35, 1039 (1987).

[26] B. L. Altshuler und Z. Pis'ma, JETP Lett. 41, 648 (1985).

[27] C. M. Marcus, R. M. Westervelt, P. F. Hopkins und A. C. Gossard, Chaos 3, 643 (1993).

[28] Y. Imry, Euro. Phys. Lett. 1, 249 (1986).

[29] B. L. Altshuler und B. I. Shklovskii, Sov. Phys. JETP 64, 127 (1986).

[30] S. Iida, H. A. Weidenmüller und J. A. Zuk, Phys. Rev. Lett. 64, 583 (1990).

[31] H. U. Baranger, R. A. Jalabert und A. D. Stone, Chaos 3, 665 (1993).

[32] I. C. Percival, J. Phys. B 6, 229 (1973).

[33] M. V. Berry und M. Robnik, J. Phys. A Math. Gen. 17, 2413 (1984).

[34] R. Ketzmerick, Phys. Rev. B 54, 10841 (1996).

[35] B. Huckestein, R. Ketzmerick und C. Lewenkopf, Phys. Rev. Lett. 84, 5504 (2000).

[36] M. Weiss, L. Hufnagel und R. Ketzmerick, submitted to Phys. Rev. Lett. (2001).

[37] R. Ketzmerick, L. Hufnagel und M. Weiss, Adv. Sol. St. Phys. 41, 473 (2001).

[38] R. Ketzmerick, L. Hufnagel, F. Steinbach und M. Weiss, Phys. Rev. Lett. 85, 1214 (2000).

[39] L. Hufnagel, R. Ketzmerick und M. Weiss, Europhys. Lett. 54, 703 (2001).

[40] L. Hufnagel, M. Weiss, A. Iomin, R. Ketzmerick, S. Fishman und T. Geisel, Phys. Rev. B 62, 15348 (2000). 
[41] L. Hufnagel, R. Ketzmerick, T. Kottos und T. Geisel, Phys. Rev. E 64, 012301 (2001).

[42] D. L. Arrowsmith und C. M. Place, Dynamical Systems (Cambridge University Press, Cambridge, 1994).

[43] A. J. Lichtenberg und M. A. Lieberman, Regular and Chaotic Dynamics (Springer Verlag, Berlin, 1992).

[44] R. Abraham und J. E. Marsden, Foundations of Mechanics (Addison-Wesley Publishing Company, New York, 1997).

[45] B. Chirikov, Phys. Rep. 52, 263 (1979).

[46] G. Casati, Chaos 6, 391 (1996).

[47] V. M. Alekseev und M. V. Yakobson, Phys. Rep. 75, 287 (1981).

[48] J. D.Meiss, Chaos 7, 139 (1996).

[49] J. N. Mather, Publ. Math. I.H.E.S. 64, 153 (1986).

[50] J. D. Meiss und E. Ott, Phys. Rev. Lett. 55, 2741 (1985).

[51] R. S. MacKay, J. D. Meiss und I. C. Percival, Phys. Rev. Lett. 52, 697 (1984).

[52] R. S. MacKay, J. D. Meiss und I. C. Percival, Physica D. 13, 55 (1984).

[53] J. D. Hanson, J. R. Cary und J. D. Meiss, J. Stat. Phys. 99, 327 (1985).

[54] R. S. MacKay, J. D. Meiss und I. C. Percival, Physica D. 27, 1 (1987).

[55] F. Haake, Quantum Chaos (Springer Verlag, Berlin, 2000).

[56] H. J. Stöckmann, Quantum Chaos (Cambridge University Press, Cambridge, 1999).

[57] S.-J. Chang und K.-J. Shi, Phys. Rev. A 34, 7 (1986).

[58] E. Wigner, Phys. Rev. E 40, 749 (1932).

[59] B. V. Chirikov und D. L. Shepelyansky, Phys. Rev. Lett. 82, 528 (1999).

[60] J. Bellissard, O. Bohigas, G. Casati und D. L. Shepelyansky, Physica D 131, ix (1999).

[61] G. M. Zaslavsky, M. Edelman und B. A. Niyazov, Chaos 7, 159 (1997).

[62] J. M. Greene, J. Math. Phys. 20, 1183 (1979).

[63] R. S. MacKay, Renormalization in Area-Preserving Maps (World Scientific, Singapore, 1993). 
[64] C. F. F. Karney, Physica D. 8, 360 (1983).

[65] R. S. MacKay, Physica D 7, 283 (1983).

[66] B. Chirikov, in Lecture Notes in Physics (Springer, Berlin New York, 1983), No. 179, S. 29.

[67] G. M. Zaslavsky und M. Edelman, Chaos 10, 135 (2000).

[68] M. F. Shlesinger und J. Klafter, Phys. Rev. Lett. 85, 2551 (1985).

[69] T. Geisel, A. Zacherl und G. Radons, Phys. Rev. Lett. 59, 2503 (1987).

[70] G. M. Zaslavsky, Lévy Flights and Related Phenomena in Physics (Springer Verlag, Berlin, 1995).

[71] G. Zumofen und J. Klafter, Phys. Rev. E 59, 3756 (1999).

[72] S. Benkadda, S. Kassibrakis, R. White und G. M. Zaslavsky, Phys. Rev. E 59, 3761 (1999).

[73] O. Bohigas, S. Tomsovic und D. Ullmo, Phys. Rep. 233, 43 (1993).

[74] E. Akkermans und G. Montambaux, Phys. Rev. Lett.. 68, 642 (1992).

[75] H. Hegger, B. Huckestein, K. Hecker, M. Janssen, A. Freimuth, G. Reckziegel und R. Tuzinski, Phys. Rev. Lett. 77, 3855 (1996).

[76] A. S. Sachrajda, R. Ketzmerick, C. Gould, Y. Feng, P. J. Kelly, A. Delage und Z. Wasilewski, Phys. Rev. Lett. 80, 1948 (1998).

[77] A. P. Micolich, R. P. Taylor, R. Newbury, J. P. Bird, R. Wirtz, C. P. Dettmann, Y. Aoyagi und T. Sugano, J. Phys. 10, 1339 (1998).

[78] Y. Ochiai, Semicon. Sci. Technol. 13, 15 (1998).

[79] Y. Takagaki, Phys. Rev. B 15, 10255 (2000).

[80] G. Casati, I. Guarneri und G. Maspero, Phys. Rev. Lett. 84, 63 (2000).

[81] Y. Alhassid, Pev. Mod. Phys. 72, 895 (2000).

[82] R. Landauer, J. Res. Dev. 1, 223 (1957).

[83] R. Landauer, Philos. Mag. 21, 863 (1970).

[84] Y. Imry und R. Landauer, Rev. Mod. Phys. 71, 306 (1999).

[85] W. H. Miller, Adv. Chem. Phys. 30, 77 (1975). 
[86] W. H. Miller, Science 233, 171 (1986).

[87] R. Blümel und U. Smilansky, Phys. Rev. Lett. 60, 477 (1988).

[88] R. A. Jalabert, H. U. Baranger und A. D. Stone, Phys. Rev. Lett. 65, 2442 (1990).

[89] Y. C. Lai, R. Blümel, E. Ott und U. Smilansky, Phys. Rev. Lett. 68, 3491 (1992).

[90] B. B. Mandelbrot, The Fractal Geometry of Nature (Freeman, San Francisco, 1982).

[91] G. Casati, G. Maspero und D. L. Shepelyansky, Phys. Rev. Lett. 82, 524 (1999).

[92] D. V. Savin und V. V. Sokolov, Phys. Rev. E 56, 4911 (1997).

[93] G. Casati, G. Maspero und D. L. Shepelyansky, Phys. Rev. E 56, 6233 (1997).

[94] T. Kottos und U. Smilansky, Phys. Rev. Lett. 70, 4794 (1997).

[95] T. Kottos und U. Smilansky, Phys. Rev. Lett. 85, 968 (2000).

[96] F. Borgonovi und I. Guarneri, J. Phys. A 25, 3239 (1992).

[97] F. Borgonovi und I. Guarneri, Phys. Rev. E 48, 2347 (1993).

[98] F. Borgonovi, I. Guarneri und L. Rebuzzini, Phys. Rev. Lett. 72, 1463 (1994).

[99] F. Bloch, Z. Phys. 52, 555 (1929).

[100] L. Landau, Z. Phys. 64, 629 (1930).

[101] P. Harper, Proc. Phys. Soc. Lond. A 68, 874 (1955).

[102] D. R. Hofstadter, Phys. Rev. B 14, 2239 (1976).

[103] T. Geisel, R. Ketzmerick und G. Petschel, Phys. Rev. Lett. 66, 1651 (1991).

[104] M. Wilkinson, Proc. R. Soc. London 391, 305 (1984).

[105] Y. Last und M. Wilkinson, J. Phys. A 25, 6123 (1992).

[106] R. Fleischmann, T. Geisel, R. Ketzmerick und G. Petschel, Physica D 86, 171 (1995).

[107] R. Lima und D. Shepelyansky, Phys. Rev. Lett. 67, 1377 (1991).

[108] T. Geisel, R. Ketzmerick und G. Petschel, Phys. Rev. Lett. 67, 3635 (1991).

[109] R. Artuso, G. Casati und D. Shepelyansky, Phys. Rev. Lett. 68, 3826 (1992).

[110] R. Artuso, G. Casati und D. Shepelyansky, Phys. Rev. Lett. 69, 3302 (1992).

[111] G. Petschel und T. Geisel, Phys. Rev. Lett. 71, 239 (1993). 
[112] I. Dana, Phys. Rev. Lett. 73, 1609 (1994).

[113] D. Springsguth, R. Ketzmerick und T. Geisel, Phys. Rev. B 56, 2036 (1997).

[114] A. Iomin und S. Fishman, Phys. Rev. Lett. 81, 1921 (1998).

[115] A. Iomin und S. Fishman, Physica D 131, 170 (1999).

[116] A. Iomin und S. Fishman, Phys. Rev. B 61, 2085 (2000).

[117] T. Geisel, R. Ketzmerick und G. Petschel, Phys. Rev. Lett. 69, 695 (1992).

[118] P. W. Anderson, Phys. Rev. 109, 1492 (1958).

[119] E. N. Economou, Green's Functions in Quantum Physics (Springer Series in Solid State Physics, Vol. 7, Springer-Verlag, Berlin, 1979).

[120] B. Huckestein und L. Schweitzer, Phys. Rev. Lett. 72, 713 (1994).

[121] I. Guarneri, Europhys. Lett. 10, 95 (1989).

[122] I. Guarneri, Europhys. Lett. 21, 729 (1993).

[123] R. Ketzmerick, K. Kruse, S. Kraut und T. Geisel, Phys. Rev. Lett. 79, 1959 (1997).

[124] I. Guarneri, J. Math. Phys. 37, 5195 (1996).

[125] I. Guarneri und H. Schulz-Baldes, Rev. Math. Phys. 11, 1249 (1999).

[126] A. Politi und S. Ruffo, Eur. Phys. J. B 14, 673 (2000).

[127] Y. F. Fyodorov und A. D. Mirlin, Int.J.Mod.Phys. 8, 3795 (1994).

[128] F. M. Israilev, T. Kottos, A. Politi und G. P. Tsironis, Phys. Rev. E 55, 4951 (1997).

[129] M. Weiss, T. Kottos und T. Geisel, Phys. Rev. B 63, 81306 (2001).

[130] H. A. Weidenmüller, Physica A 167, 28 (1990).

[131] R. Ketzmerick, K. Kruse und T. Geisel, Phys. Rev. Lett. 80, 137 (1998).

[132] M. Kohmoto, L. P. Kadanoff und C. Tang, Phys. Rev. Lett. 50, 1870 (1983).

[133] S. Ostlund, R. Pandit, D. Rand, H. J. Schellnhuber und E. D. Siggia, Phys. Rev. Lett. 50, 1873 (1983). 


\section{Danksagungen}

Prof. Dr. Theo Geisel danke ich für die Aufnahme in seine Arbeitsgruppe und die großzügige Unterstützung meiner Vorhaben. Seinem unermüdlichen Einsatz ist es zu verdanken, daß in Göttingen eine großartige Arbeitsatmosphäre mit hervorragenden Arbeitsbedingungen geschaffen worden ist.

Priv. Doz. Dr. Roland Ketzmerick und Dr. Tsampikos Kottos danke ich für die vielen Diskussionen und wertvollen Anregungen bei der Bearbeitung der verschiedenen Themen.

Matthias Weiß möchte ich für die fruchtbare Zusammenarbeit und die vielen Diskussionen danken.

Dr. Wolf-Dieter Brandt, Dr. Ragnar Fleischmann, Dr. Denny Fliegner und Yorck-Fabian Temme danke ich für ihre Hilfe im Umgang mit den Rechnern.

Ich danke den übrigen Mitgliedern der Arbeitsgruppe Steffen Arnrich, Rita Bartels, Matthias Bethge, Dmitri Bibitchkov, Agnes Bleile, Dirk Brockmann, Michael Denker, Markus Diesmann, Silke Dodel, Udo Ernst, Michael Hermann, Matthias Kaschube, Karsten Kruse, Norbert Mayer, Björn Naundorf, Alexander Ossipov, Marc-Felix Otto, Felix Petri, HansEckhard Plesser, Holger Schanz, Olaf Scherf, Michael Schnabel, Dennis Springsguth, Frank Steinbach, Tom Tetzlaff, Marc Timme, Corinna Trautsch, Fred Wolf, Alexander Zumdieck. Jeder hat seinen ganz persönlichen Anteil an der guten Atmosphäre in der Gruppe. 